\title{
WestVirginiaUniversity
}

THE RESEARCH REPOSITORY @ WVU

Graduate Theses, Dissertations, and Problem Reports

2020

\section{A Study of Impact Protection of Metacarpal Gloves}

\author{
Faisal Mohammed Alessa \\ West Virginia University, fmalessa@mix.wvu.edu
}

Follow this and additional works at: https://researchrepository.wvu.edu/etd

Part of the Ergonomics Commons, and the Industrial Engineering Commons

\section{Recommended Citation}

Alessa, Faisal Mohammed, "A Study of Impact Protection of Metacarpal Gloves" (2020). Graduate Theses, Dissertations, and Problem Reports. 7958.

https://researchrepository.wvu.edu/etd/7958

This Dissertation is protected by copyright and/or related rights. It has been brought to you by the The Research Repository @ WVU with permission from the rights-holder(s). You are free to use this Dissertation in any way that is permitted by the copyright and related rights legislation that applies to your use. For other uses you must obtain permission from the rights-holder(s) directly, unless additional rights are indicated by a Creative Commons license in the record and/ or on the work itself. This Dissertation has been accepted for inclusion in WVU Graduate Theses, Dissertations, and Problem Reports collection by an authorized administrator of The Research Repository @ WVU.

For more information, please contact researchrepository@mail.wvu.edu. 
Graduate Theses, Dissertations, and Problem Reports

2020

A Study of Impact Protection of Metacarpal Gloves

Faisal Mohammed Alessa

Follow this and additional works at: https://researchrepository.wvu.edu/etd

Part of the Ergonomics Commons, and the Industrial Engineering Commons 


\title{
A Study of Impact Protection of Metacarpal Gloves
}

\author{
Faisal Mohammed Alessa
}

Dissertation submitted to the

Benjamin M. Statler College of Engineering and Mineral Resources at West Virginia University in partial fulfillment of the requirement for the degree of

\section{Doctor of Philosophy in Industrial Engineering}

\author{
Ashish D. Nimbarte, Ph.D., Chair \\ Eduardo M. Sosa, Ph.D. Co-Chair \\ Gary Winn, Ph.D. \\ Hongwei Hsiao, Ph.D. \\ James M. Dean
}

Department of Industrial and Management Systems Engineering

\author{
Morgantown, West Virginia \\ November 2020
}

Keywords: Impact-resistant gloves, hand injuries, injury severity, cadaveric hand, surrogate hand, mining

Copyright 2020 Faisal Mohammed Alessa 


\section{Abstract \\ A Study of Impact Protection of Metacarpal Gloves}

\section{Faisal Mohammed Alessa}

Work-related wrist, hand, and finger injuries are highly prevalent in manufacturing and extractive industries. An analysis of mining-related hand injury data from over the past two decades (Alessa et al., 2020) showed that hand injuries caused by insufficient protection against impact loads (e.g. struck by accidents) were categorized with high severity. Existing literature lacks clear classification and quantification methods for the protection provided by impactresistant gloves (i.e. metacarpal gloves). A new method to establish a quantitative measure of performance for commonly used metacarpal gloves was developed and evaluated.

In the first specific aim, an experimental study using cadaveric hand specimens was performed to understand how human hand react to blunt impacts by comparing peak impact reaction forces (PRF) and number of fractures on unprotected and protected hands using two types of metacarpal gloves. The specimens were impacted at the proximal interphalangeal joints, the metacarpophalangeal joints, and the middle section of the metacarpal bones. $71 \%$ of the impacts on unprotected hands produced fractures compared to $40 \%$ for the protected hands.

In the second specific aim, surrogate hands were developed using 3D printing and gel casting techniques. The surrogate hands were calibrated and validated using the impact response data obtained from Aim \#1. The PRF values of surrogate hands were within 1 standard deviation of the cadaveric hands, with the coefficient of restitution differing by only $4 \%$. Using the surrogate hands, the protection performance of three commonly used metacarpal gloves was assessed. $77 \%$ of the impacts on unprotected hands produced fractures compared to $33 \%$ for the protected hands. PRF values for protected hands were significantly less than unprotected hands and different gloves delivered different levels of protection. Results of this study could aid safety professionals in improving their gloves selection process and could also be utilized to improve current standards for metacarpal gloves classification. Furthermore, the testing methodology and protocol presented in this research could be useful in future gloves safety studies. 


\section{Dedication}

To my loving parents, Mohammed and Haya, for their endless support and encouragement.

To my beautiful wife, Nujood, without her support and love, this work could not have been completed.

To my home country, Saudi Arabia, represented by King Saud University, for providing the opportunity and the financial support to pursue my graduate education in the United States of America. 


\section{Acknowledgments}

With utmost pleasure and privilege, I wish to take this opportunity to express my deepest gratitude to my advisor and mentor, Dr. Ashish D. Nimbarte for the continuous encouragement and support throughout the years of my studies. His guidance helped me to overcome many crisis situations and reach beyond my anticipated goals.

I would also like to express my profound gratitude to my co-advisor, Dr. Eduardo M. Sosa for being an invaluable resource during my dissertation work. This research would not have been accomplished without his willing assistance and continuous mentoring and support.

Also, a special word of thanks goes to my advisory committee: James M. Dean, Dr. Gary Winn, and Dr. Hongwei Hsiao for the insightful comments and recommendations, which incented me to widen my research from various perspectives, and provided different lenses from which to view, analyze and explain the findings.

I'm also very thankful for the assistance provided by Trevor A. Brison during the digital modeling. The assistance provided by SueAnn Woods, Amy Burt, Joy Grise, and Allison Pekar during the preparation and execution of the cadaveric hand tests is much appreciated. Also, I'm thankful to Dr. Matthew Dietz, Dr. Wayne Lambert, Suzanne Danley, Bruce Palmer, and Dr. Rosa Santos, for their assistance in the logistics necessary for the execution of the tests. Finally, the assistance provided by Dr. Jennifer Koay, Dr. Timothy Stooksberry, and Dr. Shafic Sraj for the radiological analyses is also appreciated and acknowledged. 


\section{Table of Contents}

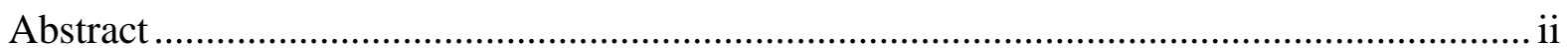

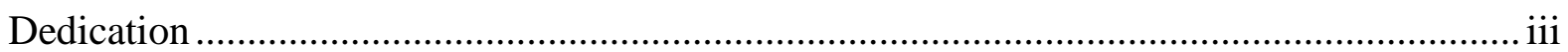

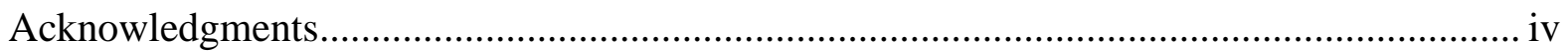

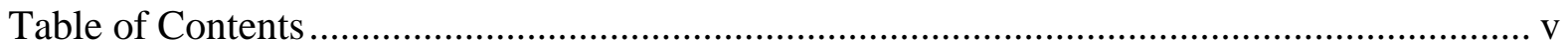

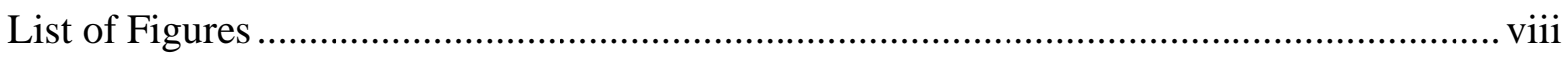

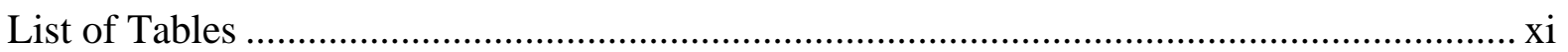

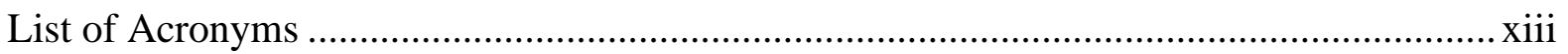

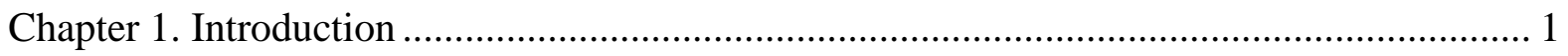

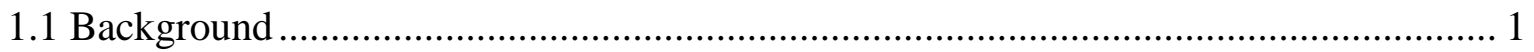

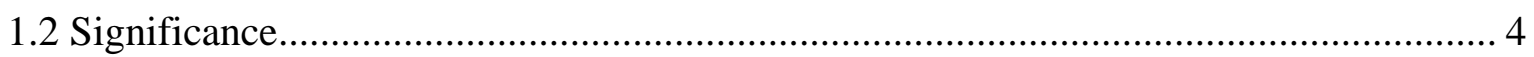

Chapter 2. Preliminary study: Incidences and severity of wrist, hand, and finger injuries in

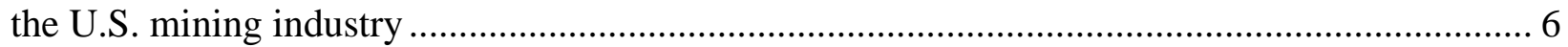

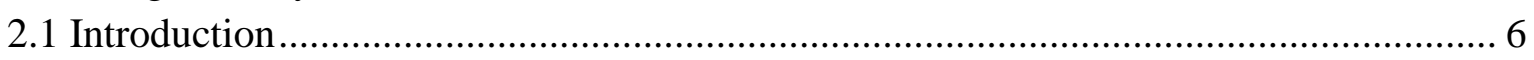

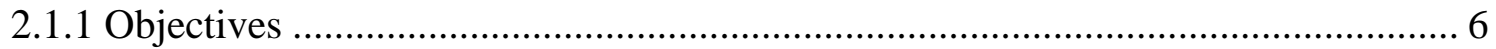

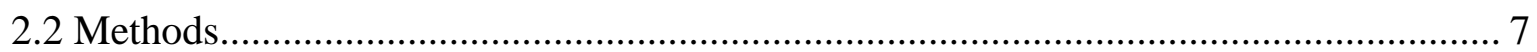

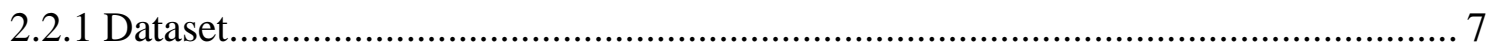

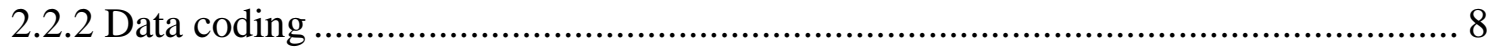

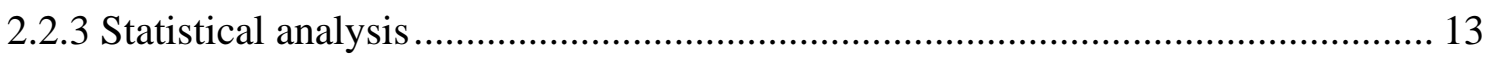

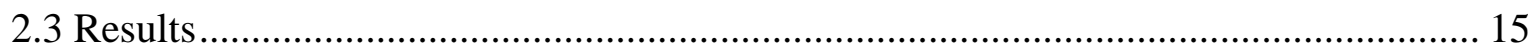

2.3.1 Number of incidents and median LWD ............................................................ 15

2.3.2 Number of incidents within severity groups ........................................................ 17

2.3.3 Injury attributes association with severity groups ............................................... 19

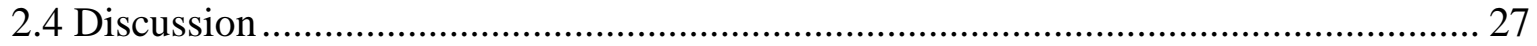

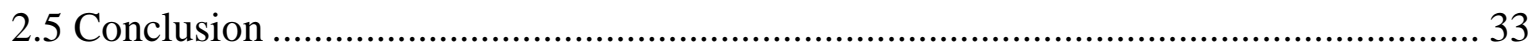

Chapter 3. Specific Aim \#1: Development and testing of impact protection measurement

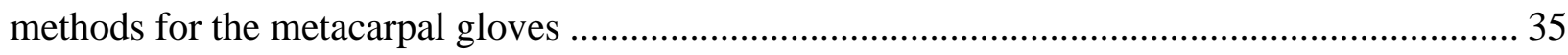

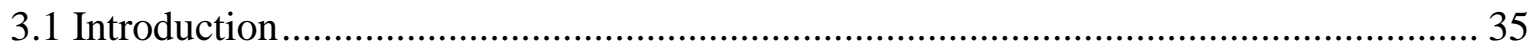

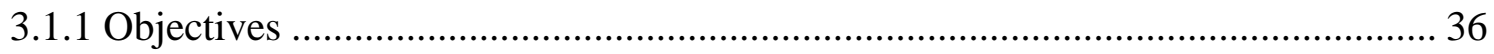

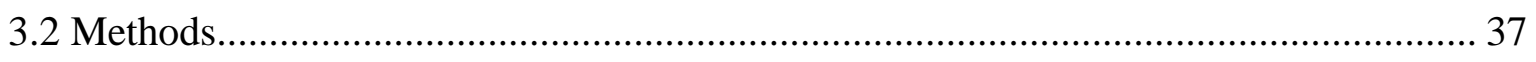

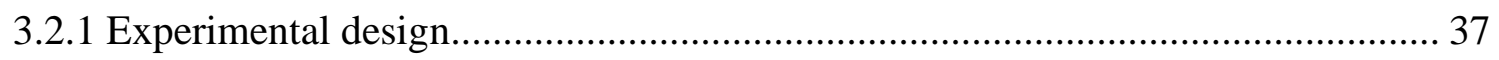

3.2.2 Testing set-up description ................................................................................ 39

3.2.3 Testing Procedure ……………………………............................................. 40

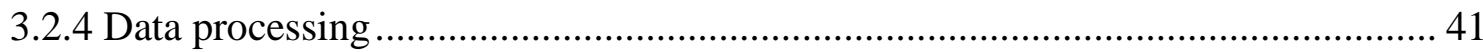




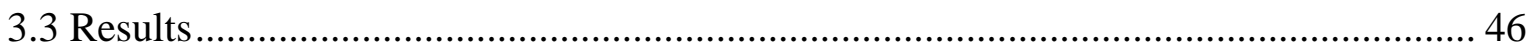

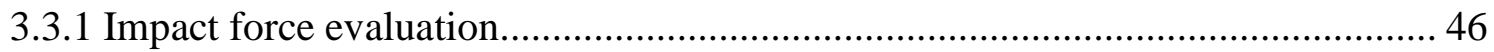

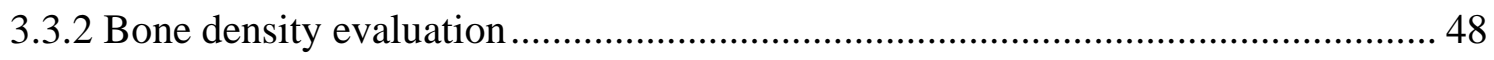

3.3.3 Fracture evaluation.......................................................................................... 49

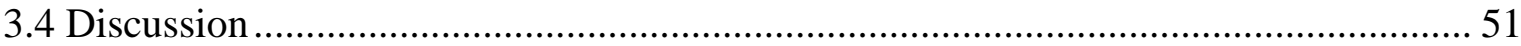

Chapter 4. Specific Aim \#2: Development of surrogate hand for performance evaluation of

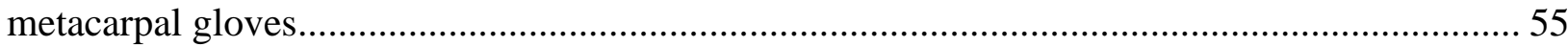

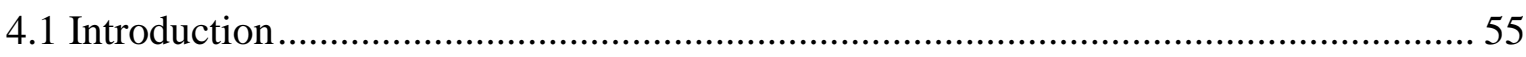

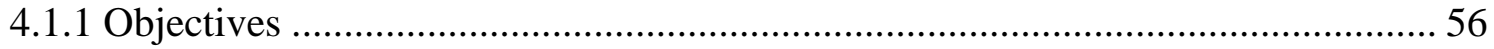

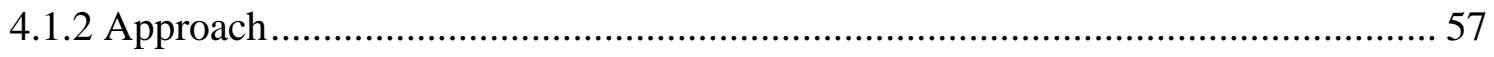

4.2 Objective 1: Design and evaluation of a surrogate hand ……………………........... 57

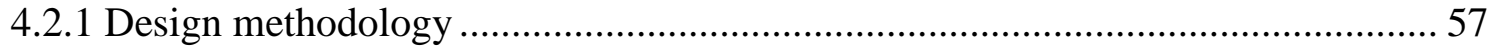

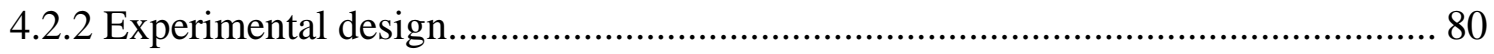

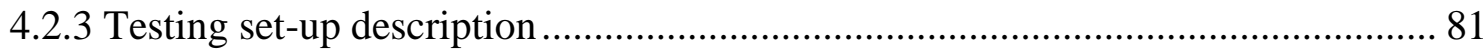

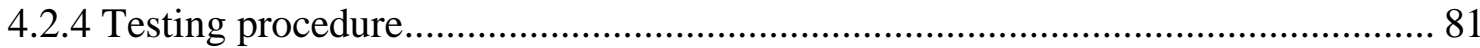

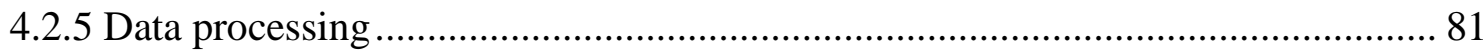

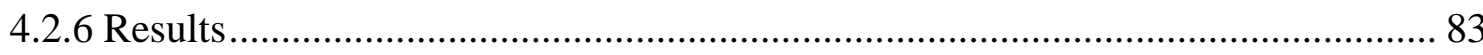

4.3 Objective 2: Glove impact resistance using surrogate hand ....................................... 85

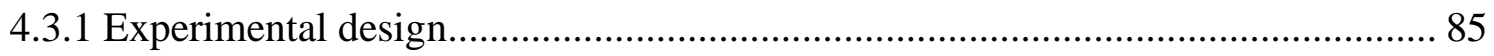

4.3.2 Gloves specifications and performance ……………….................................. 87

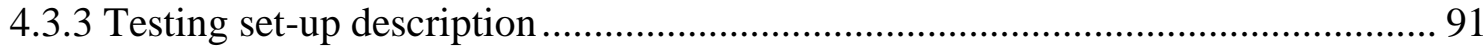

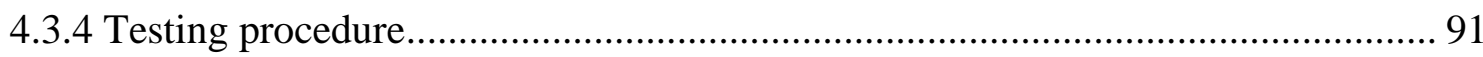

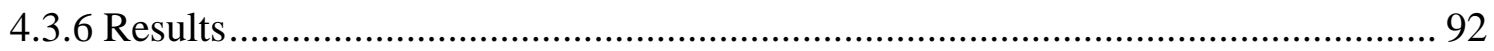

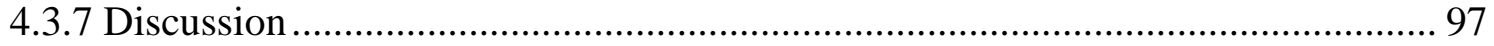

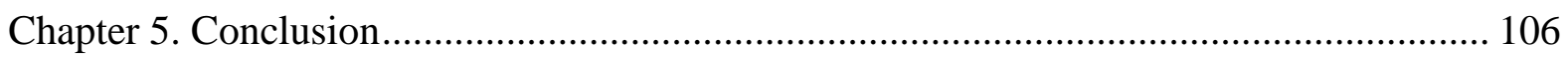

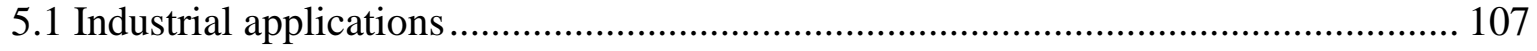

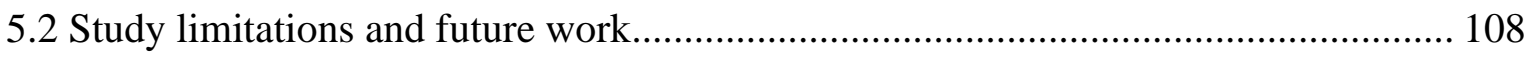

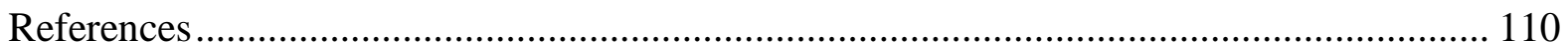

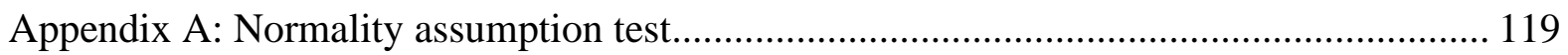

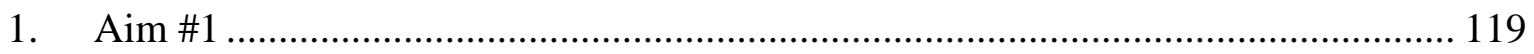

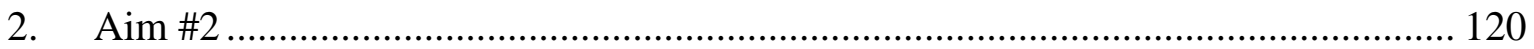

Appendix B: ANOVA tables …………………………............................................ 122 
1. ANOVA tables for Aim \#1 ............................................................................. 122

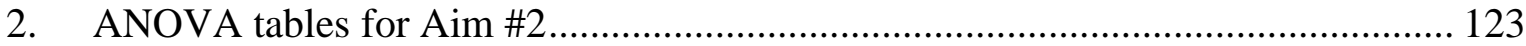

Appendix C: Datasheets............................................................................................ 125

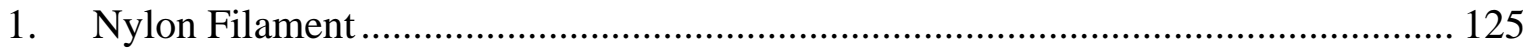

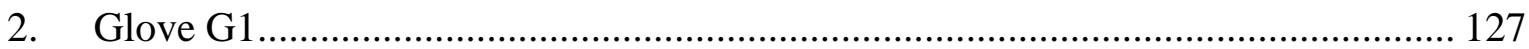

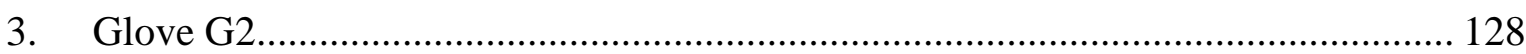

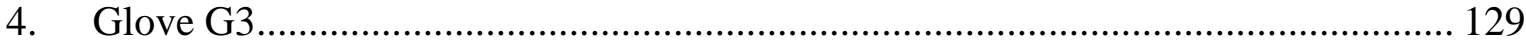

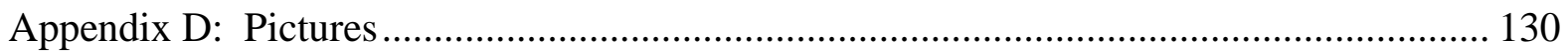

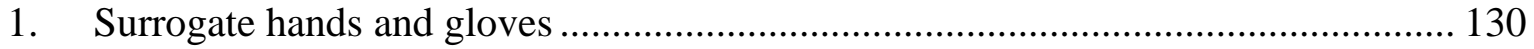

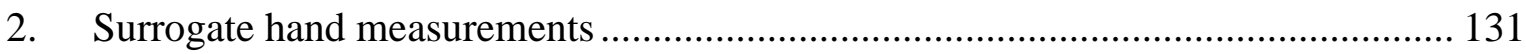

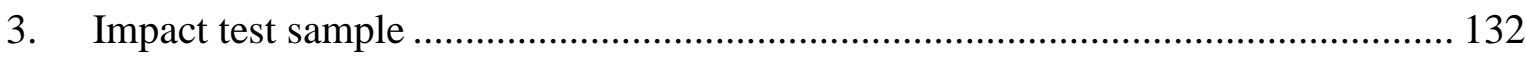

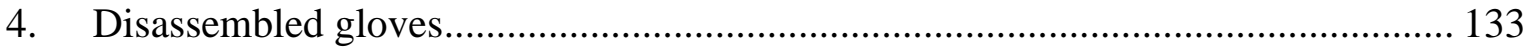




\section{List of Figures}

Figure 1: Number of NFDL, NDL incidents, and median LWD for the period from 2000 to 2017. NFDL and NDL incidents are plotted using bars and the primary axis to the left. Median LWD are plotted using line and the secondary axis to the right.

Figure 2: NFDL incidents and median LWD categorized by body parts for the period from 2000 to 2017. NFDL incidents are plotted using bars and the primary axis to the left. Median LWD are plotted using lines and the secondary axis to the right.

Figure 3: Total number of NFDL incidents and LWD for different severity groups. NFDL incidences are plotted using bars and the primary axis to the left. LWD are plotted using a line and the secondary axis to the right. LWD percentages are estimated with respect to the total LWD.

Figure 4: Yearly number of incidents for the severity groups and NDL. The secondary axis to the right is for NDL group and the primary axis to the left is for all other groups. Charts at the bottom were obtained from the normalized data and include regression equations and $\mathrm{R}^{2}$ values.

Figure 5: Number of NFDL injuries and LWD categorized by source of injuries that resulted in most injuries and LWD within maintenance activity. Note that only the main sources are included (69\% of maintenance NFDL injuries).

Figure 6: Number of NFDL injuries and LWD categorized by source of injuries that resulted in most injuries and LWD within roof bolter activities. NEC stands for "not elsewhere classified" and includes all injuries not in the main three categories. Note that only the main sources are included (95\% of roof bolter NFDL injuries).

Figure 7: Number of NFDL injuries and LWD categorized by source of injuries that resulted in most injuries and LWD within operating equipment activity. Note that only the main sources are included (66\% of operating equipment NFDL injuries).

Figure 8: Metacarpal gloves and test setup: (a) Glove G1; (b) Glove G2; (c) Components of impact testing setup (top) and testing specimens (bottom) ............................................... 38

Figure 9: Illustration of edges created by Canny filter and measurement positions............. 43

Figure 10: (a) Average total width (TW) and medullary width (MW); (b) Average cortical index (CI). Measurements made with the Canny filter and manual measurements for metacarpals M2 and M3. Bars represent standard error.

Figure 11: (a) Distribution of injurious impacts (Total fractures , $\mathrm{N}_{\mathrm{F}},=108$ ) for each Protection condition; (b) Percentage of impacts that resulted into a fracture (injurious impacts) per Position for all Protection conditions; (c) Percentage of impacts that resulted into a fracture (injurious impacts) for each Position and Protection condition. For (b) and (c), percentages were calculated from the total number of impacts in each Position for each Protection condition. ..... 50

Figure 12: A summary of the main activities performed during the digital phase. ............. 59

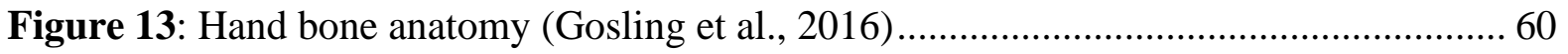

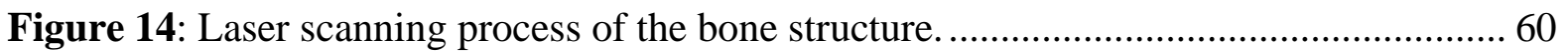




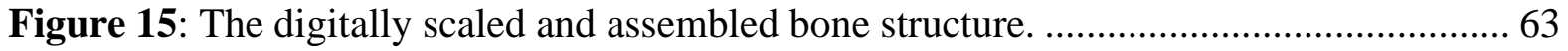

Figure 16: The scaled hand digital model.................................................................. 64

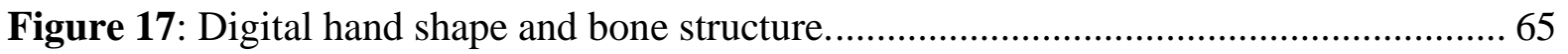

Figure 18: Hand mold model. ..................................................................................... 66

Figure 19: A summary of the main activities performed within the material selection section.

Figure 20: Heat resistance test using molten synthetic gel. Bones on the left side are without heat resistance test. Bones on the right side are after heat resistance test. ............................... 70

Figure 21: Anatomy of a long bone (OpenStax, 2017) ............................................. 71

Figure 22: A summary of the sub-sections within the manufacturing Phase section and the main activities performed. 72

Figure 23: Schematic of standard FDM machining process (Cantrell et al., 2017)............. 73

Figure 24: Slicing settings and visualization of layers build up in a slicer program........... 73

Figure 25: Cross sections of a G-code of the same bone in a slicer program with different settings of infill shape and number of perimeters. (a) Concentric infill and 5 perimeters. (b) Gyroid infill and 5 perimeters. (c) Zig Zag infill and 3 perimeters. (d) Triangular infill and 7 perimeters. (e) Solid part created by concentric perimeters and no infill

Figure 26: (a) Bone structure in slicing software; (b) 3D printed bone structure with support material (palmar view); (c) Finished 3D printed bone structure with silicon material joining

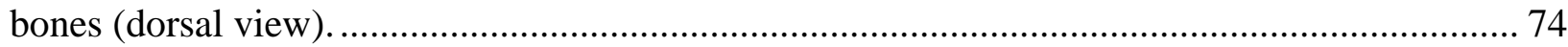

Figure 27: Initial 3D printing settings (used for Gel\#4.1 hands). .................................... 74

Figure 28: Bone structure in slicing software with the modification implemented for the surrogate hand generation Gel\#4.3. (a) Metacarpals and carpals portion; (b) Phalangeal portion.

Figure 29: A summary of the process followed during the manufacturing and development of bone structures and surrogate hands.

Figure 30: (a) 3D printed bone assembly placement on mold; (b) Palmar view of gel hand after removing one half; (c) Dorsal view of finished surrogate gel hand. ................................ 79

Figure 31: Assembled molds ready for casting right and left surrogate hands.................... 79

Figure 32: (a) The final design of the finished bone structure. (b) The casted right and left

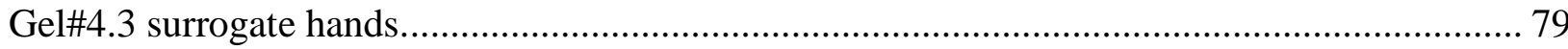

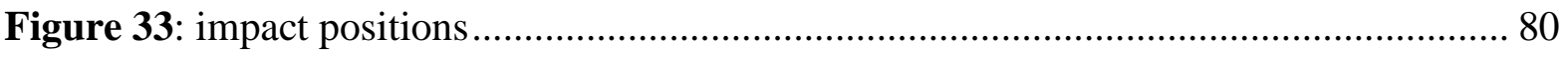

Figure 34: Schematic diagram for an impact initial drop height and rebound height for Coefficient of Restitution calculations.............................................................................. 82

Figure 35: Coefficient of restitution (COR) and energy loss (EL) values for cadaver hands $(\mathrm{CH})$ and surrogate hands manufactured with different grades of synthatic gel and using different

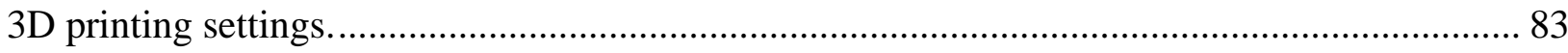

Figure 36: Metacarpal gloves considered in this study. Numbers correspond to the regions where thickness measurements were performed (see Table 18) ......................................... 87 
Figure 37: Comparisons of PRF values between full glove and only dorsal part of glove impacted directly without hands.

Figure 38: Comparisons of PRF values between conditions of gloves impacted without hand.

Figure 39: Mean values of PRF and results of the students' t-test for the effect of Protection on PRF at different levels of the independent variable Protection. A bold P-value indicates a statistically significant difference.

Figure 40: (A) Distribution of injurious impacts for each Protection condition. (B) Distribution of injurious impacts after excluding G3 data to facilitate comparisons with Aim \#1 results. (C) Distribution of injurious impacts in cadaveric hands (Aim \#1).

Figure 41: Percentage of impacts that resulted into a fracture (injurious impacts) per Position for with-glove (all gloves were combined) and no-glove Protection conditions. Percentages were calculated from the total number of impacts in each Position for each Protection condition. (a) For Gel\#4.3 surrogate hands data (Aim \#2); (b) For cadaveric hands data (Aim \#1). 96

Figure 42: Percentage of impacts that resulted into a fracture (injurious impacts) for each Position and Protection condition. Percentages were calculated from the total number of impacts in each Position for each Protection condition.

Figure A.D- 1: The different set of specimens with and without gloves used for the tests. 130

Figure A.D- 2: Measurements of circumference, length, and breadth of surrogate hands. . 131

Figure A.D- 3: Snapshots of an impact test on surrogate hand with glove showing the timeline of impact and response data.

Figure A.D- 4: Disassembled glove G1. (a) Posterior \& anterior (inner). (b) Posterior \& anterior (outer).

Figure A.D- 5: Disassembled glove G2. (a) Posterior. (b) Anterior.................................. 134

Figure A.D- 6: Disassembled glove G2. (a) Posterior. (b) Anterior.................................. 135 


\section{List of Tables}

Table 1: Summary statistics for wrist, hand, and finger incidents.

Table 2: Summary statistics and crosstabs for severity groups and accident type, nature of injury, and activity variables.

Table 3: Summary statistics and crosstabs for severity groups and experience, subunit, and injured finger variables.

Table 4: Results of ANOVA test for the effect of Protection on PRF. Bold P-value indicates a statistically significant difference.

Table 5: Results of ANOVA test for the effect of Position on PRF. Bold P-value indicates a statistically significant difference.

Table 6: Summary statistics, Results of the students' t-test for the effect of Protection on PRF. Bold P-value indicates a statistically significant difference.

Table 7: Summary of average PRF, TPRF, KE, and IPI for each glove type compared to their pairs of no-glove tests.

Table 8: Tested specimens' information. Donor age, hand length \& breadth (measured from X-ray images), average values of second and third metacarpal TW, MW, CT, and CI for each specimen, and total number of fractures after impact.

Table 9: Length and breadth of $50^{\text {th }}$ percentile male hand (obtained from literature) and measured from Aim \#1 study for 5 selected cadaver specimens.

Table 10: Length and breadth of 5 selected cadaveric specimens from Aim \#1 study.

Table 11: Synthetic gel proprieties. Shore rating is on the Shore 00 standard (Humimic Medical, Fort Smith, AR).

Table 12: Results of the heat resistance test using molten synthetic gel on 3D printed bones using different materials.

Table 13: summary of 3D printing settings used in printing bone structures of different generations of surrogate hands.

Table 14: The percentage mean difference in peak reaction force ( $\% \Delta$ PRF) between cadaver hands $(\mathrm{CH})$ and different generations of Gel \#4 surrogate hands. Difference columns compare former column to cadaver hand column. Bolded values are within 1 standard deviation of cadaver hand values.

Table 15: Weight, length, breadth, and circumference of all tested surrogate hands with noglove and with all types of considered gloves.

Table 16: Length, breadth, and circumference of $50^{\text {th }}$ percentile male hand (obtained from literature).

Table 17: Layers and material types for the tested gloves at different regions. Region numbers are illustrated in Figure 36. 88

Table 18: Means of material layers thicknesses for the tested gloves at different regions. Region numbers are illustrated in Figure 36. 
Table 19: Results of ANOVA test for the effect of Protection on PRF. Bold P-value indicates a statistically significant difference.

Table 20: Results of ANOVA test for the effect of Position on PRF. Bold P-value indicates a statistically significant difference.

Table 21: Summary of average PRF, TPRF, and KE values; and PRF values change between the level "no-glove" and the evaluated gloves across all levels of Protection and Position........ 94

Table 22: Values of IPI compared to Aim \#1 study and previously reported data.............. 94 


\section{List of Acronyms}

$\begin{array}{ll}\text { ANOVA } & \text { Analysis of variance } \\ \text { BL } & \text { Bone length } \\ \text { BMD } & \text { Bone mineral density } \\ \text { COR } & \text { Coefficient of restitution } \\ \text { COV } & \text { Coefficient of variance } \\ \text { CI } & \text { Cortical index } \\ \text { CT } & \text { Cortical thickness } \\ \text { EL } & \text { Energy loss } \\ \text { FR } & \text { Force reduction } \\ \text { Hz } & \text { Hertz } \\ \text { IPI } & \text { Impact protection index } \\ \text { J } & \text { joule } \\ \text { KE } & \text { Kinetic energy } \\ \text { kg } & \text { Kilogram } \\ \text { kN } & \text { Kilo Newton } \\ \text { LWD } & \text { Lost workdays } \\ \text { m } & \text { Meter } \\ \text { mm } & \text { Millimeter } \\ \text { MCP } & \text { Metacarpophalangeal } \\ \text { MSHA } & \text { The Mine Safety and Health Administration } \\ \text { MW } & \text { Medullary width } \\ \text { N } & \text { Newton } \\ \text { NDL } & \text { Nonfatal injuries with no days lost } \\ \text { NFDL } & \text { Nonfatal injuries with days lost } \\ \text { PIP } & \text { Proximal interphalangeal } \\ \text { PPE } & \text { Personal protective equipment } \\ \text { PRF } & \text { Peak reaction force } \\ \text { SD } & \text { Standard Deviation } \\ \text { TPR } & \text { Thermoplastic rubber } \\ \text { TPRF } & \text { Peak reaction force transferred } \\ \text { TW } & \text { Total width } \\ \text { WVU } & \text { West Virginia University } \\ & \end{array}$




\section{Chapter 1. Introduction}

\subsection{Background}

Work-related hand injuries remain one of the most prevalent injuries in many industries. In 2015, wrist, hand, and finger injuries were the leading cause of emergency department injury visits in the US (Rui \& Kang, 2015) and the wrist, hand, and finger were the most affected body parts in 2017, resulting in 157,060 lost workday cases (Bureau of Labor Statistics, 2017b). A substantial proportion of these injuries is linked to the mining industry, which is considered one of the most hazardous occupations (Paul, 2009; Ural \& Demirkol, 2008). According to the U.S. Bureau of Labor Statistics, the median lost workdays in the mining industry in 2017 was the highest (i.e. 32 days) compared to other industries (Bureau of Labor Statistics, 2017a). Hazards in the mining industry stem from the nature of mining activities and surrounding environment. Mine workers engage in several hazardous activities such as operating and maintaining heavy equipment including excavators, large bulldozers, transportation trucks, and roof bolter machines. Mining tasks also involve dealing with several powered (e.g. electric saw and drill) and non-powered hand tools (e.g. axe, hammer, and wrench). Performance of such tasks is more hazardous when coupled with the risks of falling rocks, and the noisy, poorly ventilated and lighted surrounding environment.

Laflamme \& Blank (1996) evaluated injuries in the Swedish underground mines between 1980 and 1993 and found that wrist, hand, and finger were the highest affected body parts (28\%). Another study evaluated maintenance and repair injuries in US mining from 2002 to 2011 and reported that during activities that involve material handling, use of powered and non-powered hand tools and the machinery, hand and finger injuries accounted for more than third of the 
injuries (Pollard et al., 2014). In most of the cases, recovery time for injured workers was fairly short. However, a relatively large proportion of these injuries require long recovery and rehabilitation periods, and some even lead to permanent or partial disability.

The structure of the human hand is complex and consists of multiple types of tissues including bones, muscles and ligaments. Such complexity of the hand allows for a wide range of functional capabilities (Abraham \& Scott, 2010). However, injury to this intricate structure could be medically expensive (Putter et al., 2012) and may result in several days away from work or workdays with restricted activity (Eisele et al., 2018; Sorock et al., 2001). A recent cost-ofillness literature review concluded that the median total cost (i.e. direct, indirect, and intangible) per case of hand injury is US $\$ 6,951$, with an interquartile range of $\$ 3,357-\$ 22,274$ (Robinson et al., 2016). Another study suggested that nearly $75 \%$ of the total cost of hand and wrist injuries is attributed to productivity loss, which is primarily caused by lost workdays (De Putter et al., 2016).

In spite of the high burden of hand injuries in mining industry, the literature lacks relevant and latest data and/or trends on such injures. Therefore, a preliminary study was conducted by performing an exhaustive injury data analysis of wrist, hand, and finger injuries in the mining industry, which is presented in detail in Chapter 2. In this study, the trends and changes of rates and severity of hand injuries in the U.S. mining industry over the past two decades were investigated. The underlying circumstances and factors leading to increased severity and number of hand injuries among mine workers were also identified. One of the conclusions of this study was that the impact related hand injuries were highly prevalent in the mining industry and significantly contributed to number of lost workdays. Therefore, as a safety mechanism, many 
workers wear impact protective gloves (i.e. metacarpal gloves) as personal protective equipment (PPE) to protect against impact hazards.

About forty-five glove manufacturers are operating just in North-America and many more around the world. Each manufacturer produces and supplies several models of industrial gloves (Dolez et al., 2010); however, not all of them are suitable for all industries and applications. Many of those suppliers offer gloves with features designed to protect workers against an individual or a combination of several hazards including: mechanical protection (which requires cut, puncture, and abrasion resistance); chemical protection (requiring chemical permeation and chemical degradation resistance, and detection of holes); heat and flame protection (requiring flame and conductive heat resistance, and heat degradation) and protection from cold, often following performance recommendations outlined in standards such as the ANSI/ISEA 105-2016 or the EN388, and more recently the ANSI/ISEA 138-2019.

The recently released ANSI/ISEA 138-2019 standard does not require the utilization of any type of hand, real or surrogate, to evaluate the performance of metacarpal gloves. The standard establishes three levels of performance scale based merely on forces measured during direct impacts at different locations (fingers, thumb, and knuckles, but not metacarpals) of the glove under consideration, neglecting the presence of the hand. For an impact energy of $5 \mathrm{~J}$, the impact resistance is divided into three performance levels: Level 3 (the highest performance level), Level 2, and Level 1 (the lowest performance level). The corresponding mean transmitted impact forces are $\leq 4 \mathrm{kN}, \leq 6.5 \mathrm{kN}$, and $\leq 9 \mathrm{kN}$, respectively. The standard does not include a rationale behind these values nor consider higher levels of impact energy which are common in the mining industry. The inclusion of hand stiffness in the tests, or an indicator that includes glove and noglove conditions would allow for more accurate protection quantification. Such knowledge could 
be utilized for better identification and selection of suitable gloves for the different activities of the end users. This research aims to simulate realistic impact test with the use of cadaveric specimens to develop a tool that provides a realistic quantification of impact forces that can be useful to develop and implement a surrogate hand that replicates biomechanical properties of human hand.

\subsection{Significance}

Our preliminary study concluded that while the total number of hand injuries declined over the past two decades, injury severity increased dramatically. Factors which often involve impact related hazards (e.g. struck by and caught in accidents, and fracture and amputation injuries) were associated with the severe injuries. Mine workers wear impact protection gloves (i.e. metacarpal gloves) to protect against impact hazards; however, literature lacks guidance/criteria regarding selection methods of the metacarpal gloves for a given task. Therefore, this study was aimed at developing and testing newer methods to establish a quantitative measure of performance for commonly used metacarpal gloves. It was hypothesized that the commercially available metacarpal gloves may not provide identical protection against impact loads and therefore, cannot be suitable for all applications. The rationale for the current study is that the quantitative measure of performance could allow for better identification and selection of suitable gloves for different activities carried out by mine workers. To test the hypothesis, two specific aims were completed:

\section{Specific Aim \#1, to develop and test the impact protection measurement methods for} the metacarpal gloves: an experimental study using cadaveric hand specimens was performed to compare peak impact reaction forces and number of fractures on unprotected and protected hands for selected metacarpal gloves. In this aim, the testing methods were validated and a basic 
comparison matrix for selected metacarpal gloves was generated. The insights gained regarding the response of human hand structures to blunt impacts were used in the Aim \#2 to validate and improve the designs of a surrogate hands.

\section{Specific Aim \#2, to develop scale of performance for commonly used metacarpal gloves:}

surrogate hands were developed using 3D printing and gel casting techniques. The designs of surrogate hand specimens were validated using the data from Aim \#1. Impact protection measurements (same as Aim \#1) and a comparison matrix were developed for commonly used metacarpal gloves. The comparison matrix can assist the mine safety professionals in improving their glove selection process. 


\section{Chapter 2. Preliminary study: Incidences and severity of wrist, hand, and finger injuries in the U.S. mining industry}

\subsection{Introduction}

Several attempts have been made to better understand the causes and effects of workplace injuries in the mining industry. Overall, the majority of previous literature assessed the tasks with elevated risk of injury (e.g. maintenance, roof bolting, and operating equipment), contributions of environmental factors (e.g. underground mines), characteristics of vulnerable workers (e.g. age and experience), accident types (e.g. fall and caught in), and most-affected body parts (Margolis, 2010; Moore et al., 2009; Nasarwanji et al., 2018; Pollard et al., 2014; Sammarco et al., 2016; Santos et al., 2010). Other studies explored injury risks predictors (Javadi et al., 2017; Jian et al., 2009) and injury prevention methods (Breuer et al., 2002). Pollard et al. (2014) evaluated maintenance and repair injuries in the American mining industry, concluding that a significant association exists between maintenance tasks and the number of reported hand and finger injuries. A prior study on hand injuries in mining industry was performed over thirty years ago, using a rather limited sample size (58 samples) by Morgan \& Harrop (1985). The authors investigated hand injuries in South Wales mines, reporting that hand crushes and fractures were the most common reported injuries, caused primarily by falling rocks or coals.

\subsubsection{Objectives}

Despite a high prevalence of hand injuries in mining industry, our knowledge regarding the number, type, causation and severity of these injuries is limited. Gaining such knowledge is critical for future injury prevention and intervention programs. Therefore, the purpose of this preliminary study was to analyze the yearly trends of wrist, hand, and finger injury data obtained 
from the Mine Safety and Health Administration (MSHA) for the period from 2000 to 2017. Specific study objectives were: (1) to explore the changes in number and severity of hand injuries over the past 18 years. Based on the guidance from previous studies, the severity was estimated using median lost workdays (Grayson et al., 1998; Lowery et al., 2000; B. NowrouziKia et al., 2017; Behdin Nowrouzi-Kia et al., 2018; Sammarco et al., 2016); and (2) to identify the circumstances and attributes associated with injury severity.

\subsection{Methods}

\subsubsection{Dataset}

In accordance with Title 30, Part 50 of the U.S. Code of Federal Regulations (30 CFR 50.20), mine operators and contractors in the U.S. are required by MSHA to document all reportable accidents, injuries, and illnesses using the form MSHA 7000-1. The data is then compiled by MSHA and is available at https://arlweb.msha.gov/OpenGovernmentData/OGIMSHA.asp. In this study, eighteen years of MSHA accident, injury and illness data from 2000 to 2017 were initially considered for the analysis $(n=222,576)$. The dataset was filtered based on the injured body part to extract wrist, hand, and finger injuries $(n=47,903)$. The dataset was reduced further to include cases which resulted in MSHA Degree of Injury Codes 2-6: total or partial permanent disabilities, actual days away from work and/or days of restricted work activity, and no days away from work nor days of restricted work activity $(n=45,509)$. This reduction resulted in exclusion of all cases that do not fall within the scope of this study including cases which resulted in MSHA Degree of Injury Codes 0-1 and 7-10: reportable incidents not associated with an injury, fatalities, illnesses, cases due to natural causes, cases involving nonemployees, and cases determined by MSHA to be nonchargeable. Finally, data pertaining to office workers' 
injuries and cases with missing critical information (e.g. missing number of lost workdays) were removed which resulted in the final dataset consisting of 45,179 cases of mining injuries.

\subsubsection{Data coding}

The final dataset was classified using MSHA Degree of Injury Codes into two main categories: nonfatal injuries with days lost ("NFDL") and injuries with no days lost ("NDL"). NFDL includes cases which resulted in total or partial permanent disabilities (MSHA Degree of Injury Code 2), actual days away from work (MSHA Degree of Injury Code 3) and/or days of restricted work activity (MSHA Degree of Injury Codes 4 and 5). NDL includes cases with no days away from work nor days of restricted work activity (MSHA Degree of Injury Code 6). The dataset also includes several other variables such as mine ID, mining equipment, injured occupation, etc. For the purpose of this study, ten relevant variables were identified: accident date (year), injured body part, accident type, nature of injury, lost workdays (LWD), activity, subunit, experience (total), source of injury, and narrative. The accident date variable includes 18 levels of years, which are the years from 2000 to 2017. The injured body part variable includes three levels of body parts, the wrist, hand, and finger. Levels for the remaining variables are explained in subsequent sections.

\subsubsection{Accident type}

MSHA categorizes all cases by "accident type" to identify events which result in an accident or reported injury. As a result, the initial assessment of the dataset showed 35 levels for accident type. In the current study, cases categorized as "the worker being struck by or striking an object" were grouped into the struck by category (MSHA Accident Type codes 1-8). Cases caused by "the worker falling" were grouped into the fall category (MSHA Accident Type codes 9-19). The category caught in combined all cases where "the worker was caught in, under, or between an 
object or objects" (MSHA Accident Type codes 20-24). Cases resulting from "an excessive physical effort" were grouped into the overexertion category (MSHA Accident Type codes 2730). The remaining accident types that account for less than $2 \%$ of all cases and are not directly related to physical activities were grouped into the other category. Thus, the "accident type" variable included five levels: struck by, fall, caught in, overexertion, and other.

\subsubsection{Nature of injury}

The nature of the injury is used to identify the injury in terms of its principal physical characteristics. The initial assessment of the dataset included 18 nature of injury levels such as amputation, fracture, sprain/strain, etc. Within accident types, different nature of injury levels were assessed, and only levels which accounted for more than $2 \%$ of the cases within an accident type (excluding other) were considered in the analysis. The remaining nature of injury levels were grouped into the other natures level. Thus, the "nature of injury" variable included ten levels: amputation, contusion/bruise, crushing, cut/laceration, dislocation, fracture, joint inflammation, sprain/strain, multiple injuries, and other natures.

\subsubsection{Activity}

The "activity" variable categorizes incidents based on the specific activity the worker was performing at the time of the incident. The initial assessment of the dataset revealed 95 work activity levels. Similar activities were grouped into a single level. Operating equipment activities (MSHA Activity codes 44-73) were grouped into the operating equipment level, and roof bolting activities (MSHA Activity codes 77-80) were combined into the roof bolter level. Activities that account for less than $2 \%$ of the cases within an accident type (excluding other) were grouped into the other activities level. In total eleven levels were considered for the variable "activity": maintenance, handling material, handling coal/rock/ore, hand tools (powered), hand tools (not 
powered), roof bolter, operating equipment, getting on/off equipment, walking/running, climb scaffolds/ladders, and other activities.

\subsubsection{Experience}

Work experience for injured persons was expressed as a decimal of the number of years and months ("year. month"). The initial assessment of the dataset showed that the distribution of this variable (both NDL and NFDL data) was a long-tailed positive-skewed distribution with a median value of 5 years (IQR 1.23 -15) and a range from 0-58 years. Seven levels for the variable "experience" were considered in the current analysis: [0-5], [6-10], [11-15], [16-20], [21-25], [26-30], and [>30] years.

\subsubsection{Subunit}

A "subunit" is a location within a mine where an accident has occurred. An initial assessment of the dataset revealed 9 levels for this variable. Subunit levels were assessed within accident type and the levels which accounted for more than $2 \%$ of the cases within an accident type (excluding other) were included in the analysis. Levels with less than $2 \%$ of the cases were categorized as other subunits. Thus, five levels were used for the variable "subunit": underground, surface at underground, Strip/Quarry/Open pit, mill operation, and other subunits.

\subsubsection{Lost workdays (LWD)}

The dataset includes three different "lost workday" variables: the number of actual days lost from work due to worker absenteeism for no less than one day, the number of days of restricted work activity after returning to work, and the number of scheduled charges. "Scheduled charges" ("statutory days") is a uniform system that assigns values of LWD for fatalities, as well as permanent, partial or total disabilities such as amputation (Coleman \& Kerkering, 2007). Scheduled charges values were developed as an attempt to quantify the future productivity loss 
caused by death or disability (MSHA, 1986). The current analysis computed number of LWD by taking the maximum of either the statutory days, or the sum of the actual days lost plus the days of restricted work activity. This method of LWD calculation is based on recommendations from the National Institute of Occupational Safety and Health (NIOSH) on standard statistical methodology (https://www.cdc.gov/niosh/mining/statistics/methodology.html), as well as the work of Sammarco et al. (2016).

The distribution of the calculated LWD was represented by a long-tailed positive-skewed distribution with a low mean value; thus, median LWD was used as a severity measure in the current study. A similar approach was often used in the literature (B. Nowrouzi-Kia et al., 2017; Behdin Nowrouzi-Kia et al., 2018; Sammarco et al., 2016). The overall median LWD was 18 days (IQR 6-47) with a range from 1 to 3,900. Six levels for the "lost workdays" variable based on amount of time lost or severity of the injury were used in the current analysis, and were grouped by severity: [1-5], [6-10], [11-20], [21-30], [31-60], and [>60] LWD.

\subsubsection{Injured finger (narrative analysis)}

The MSHA database provided a short narrative description of each reported accident, injury or illness. The narrative for finger NFDL injuries $(14,306)$ were evaluated to extract the name of the injured finger, if reported. This analysis was mainly performed using the Matlab Text Analytics Toolbox (2019b). The first step in this analysis involved cleaning the text data and identifying misspelled words by comparing each word in the selected narrative to a vocabulary of known words. The list of misspelled words was then read and only words which referred to naming fingers were corrected, as the list of misspelled words was lengthy. Next, the language used in the narrative for naming the injured fingers was examined. This step involved reading multiple lines, visualizing the distribution of text using word clouds graphs and word frequency 
tables, and searching the data using keywords of fingers names. This step yielded a list of all possible keywords used for naming injured fingers in the narrative, as each finger could have several names (e.g. "index finger" is also refered to as the "forefinger", "pointer", "trigger finger", "digitus secundus", etc.).

The identified keywords were then used to search the narrative for the names of injured fingers in two consecutive steps. The first step involved searching with the word "finger" added to the keywords (e.g. "ring finger") and classifying the injured finger in identified cases. After excluding the classified cases in step one from the dataset, a second iteration was performed on unclassified cases, using only the keywords for searching without the word "finger" (e.g. "ring") and reviewing all results to look for irrelavent uses of the identified keywords. These two steps allowed for filtering of irrelevent cases, as some keywords could be used with different meanings (e.g. "ring finger" vs. "lock ring"). This analysis resulted in classifying 9,020 NFDL finger cases. Among the remaining 5,286 unclassified cases, a word frequency table was created for words repeated more than twice, and searched for possible missed keywords. This final analysis resulted in classification of 16 more cases, increasing the total classified cases to 9,036 and resulting in 5,270 cases of NFDL finger injuries with unclear or unreported injured finger type. Results of this analysis was used to create the variable "injured finger" with the folowing levels: thumb, index, middle, ring, little and multiple. The level multiple included cases with more than one injured finger.

\subsubsection{Source of injury}

MSHA categorizes all cases by "source of injury" to identify the object, substance, exposure or bodily motion which directly caused the reported injury. Analysis of the main sources of injury was performed for activities that caused the NFDL injuries with the highest severity. Such 
analysis is useful in providing further details regarding the performed tasks at the time of injury which could guide future prevention efforts.

\subsubsection{Statistical analysis}

First, the median LWD per year was calculated and examined to understand yearly changes in the number of incidents. Next, the injury dataset was categorized into seven groups based on severity which include non-severe NDL injuries, and six levels of the LWD variable (see Section 2.2.2.6). Within each severity group, the data were analyzed to understand yearly trends via regression with respect to time (year). To facilitate comparison between different severity groups, the yearly data within each severity group were normalized with respect to the total number of injuries within that group.

The next step of statistical analysis involved evaluation of association between the severity groups and all other variables using a chi-squared test. For associations that were statistically significant, a chi-squared post-hoc analysis was performed using the adjusted standardized residuals method with the Bonferroni correction. The findings are summarized using crosstabs (Table 2 andTable 3). The top row in the crosstab tables indicates various severity groups and the first column lists levels of other variables. Thus, cells in the crosstab tables represent the number of incidences for different severity groups corresponding to various levels of the variables. The contribution of each cell for significantly related variables was assessed as significantly higher or lower based on the expected value for that cell (Bewick et al., 2003; MacDonald \& Gardner, 2000). Cells with significantly higher than expected values falling under high severity groups [21-30], [31-60], and [>60] indicate an elevated injury severity. The Kolmogorov-Smirnov test was used to assess the data fit to the normal distribution and level of significance was set at 0.05. 


\subsubsection{Severity index}

The post-hoc analysis based on adjusted standardized residuals compares observed values of incidents with expected values, both of which have the same marginal distributions (row and column totals). Therefore, such analysis is not very sensitive to the column proportion, the contribution of a cell to the total number of incidents within a severity group. As a result, a severity index (SI) was used complementarily, which incorporates the contribution of a cell to the total number of incidents within a severity group and resulted LWD. The SI was adopted and modified from the work of Grayson et al. (1998):

Severity Index $(S I)=\left[\sum_{i=1}^{n} \frac{N F D L_{i}}{C T_{i}} \times W_{i}\right] \times\left[\frac{L W D}{N F D L}\right]$

Where $i$ represent severity group, $\mathrm{i}=1$ to 6 ;

$C T_{i}$ is the column total of a severity group;

$W_{i}$ is the weight assigned for a severity group.

$W_{i}=L W D_{i} / L W D_{\text {Total }}$

$L W D_{i}$ is the number of LWD for severity group i.

$L W D_{\text {Total }}$ is the total LWD resulted from all NFDL injuries $(938,467)$

The, weights for the severity groups [1-5], [6-10], [11-20], [21-30], [31-60], and [>60] were $.02, .03, .05, .06, .19$, and .65 , respectively. This is further explained in Section 2.3.2 and Figure 3. 


\subsection{Results}

\subsubsection{Number of incidents and median LWD}

From 2000 to 2017 , there were a total of 45,179 wrist, hand, and finger NFDL and NDL incidents. These incidents resulted in 938,467 LWD, which is equivalent to nearly 208 personyears lost annually. The overall distribution of the total incidents between the assessed body parts were 9\%, 22\%, and 69\% for the wrist, hand, and finger, respectively (Table 1). Wrist cases were associated with the highest median LWD (24), and finger cases were associated with the highest cumulative LWD $(616,696)$.

Table 1: Summary statistics for wrist, hand, and finger incidents.

\begin{tabular}{ccccccc}
\hline Body part & NDL (\%) & NFDL (\%) & Total (\%) & LWD (\%) & $\begin{array}{c}\text { Mean } \\
\text { LWD }\end{array}$ & $\begin{array}{c}\text { Median LWD } \\
(\text { IQR })\end{array}$ \\
\hline Finger & $16,857(73)$ & $14,306(65)$ & $31,163(69)$ & $61,6696(66)$ & 43 & $19(42)$ \\
Hand & $4,926(21)$ & $4,924(22)$ & $9,850(22)$ & $187,485(20)$ & 38 & $12(32)$ \\
Wrist & $1,352(6)$ & $2,814(13)$ & $4,166(9)$ & $134,286(14)$ & 48 & $24(55)$ \\
\hline
\end{tabular}

Figure 1 shows the overall yearly trends of NFDL and NDL incidents and the corresponding median LWD. The total number of incidents during the year 2000 (sum of NDL and NFDL) was 3,550 incidents, which is more than double the total incidents in 2017 (1,551 incidents). In general, the number of NFDL and NDL incidents exhibited fluctuating downward trends, with NDL incidents greater than NFDL incidents for most years except 2000, 2002 and from 2014 to 2017. The yearly trend of median LWD was slightly flat, with some fluctuation from 2000 to 2006. The yearly trend then increased from 2006 through 2017. The minimum and maximum median LWD were 14 in 2001 and 2003, and 25 in 2014 and 2015 respectively. The largest increments of the median LWD occurred from 2008 to 2009, 2010 to 2011, and from 2013 to 2014. 
Figure 2 shows the overall yearly trends in NFDL and median LWD data when organized by body parts (wrist, hand, and finger). Yearly changes in number of NFDL incidents for all three body parts exhibited downward fluctuating trends with similar patterns. The number of NFDL incidents during the first and last years of the examined period showed reductions by $56 \%, 62 \%$ and $52 \%$ for the wrist, hand, and finger, respectively. Although median LWD exhibited an overall increasing trend for all three body parts, some differences in the overall pattern as well as the amount of increment were observed. While the median LWD trend of wrist injuries followed an increasing pattern with big year-to-year fluctuations, for finger injuries the LWD trend rose steadily. The LWD trend for hand injuries, on the other hand, remained constant from 2000 to 2008, but then increased with some fluctuation from 2008 to 2017.

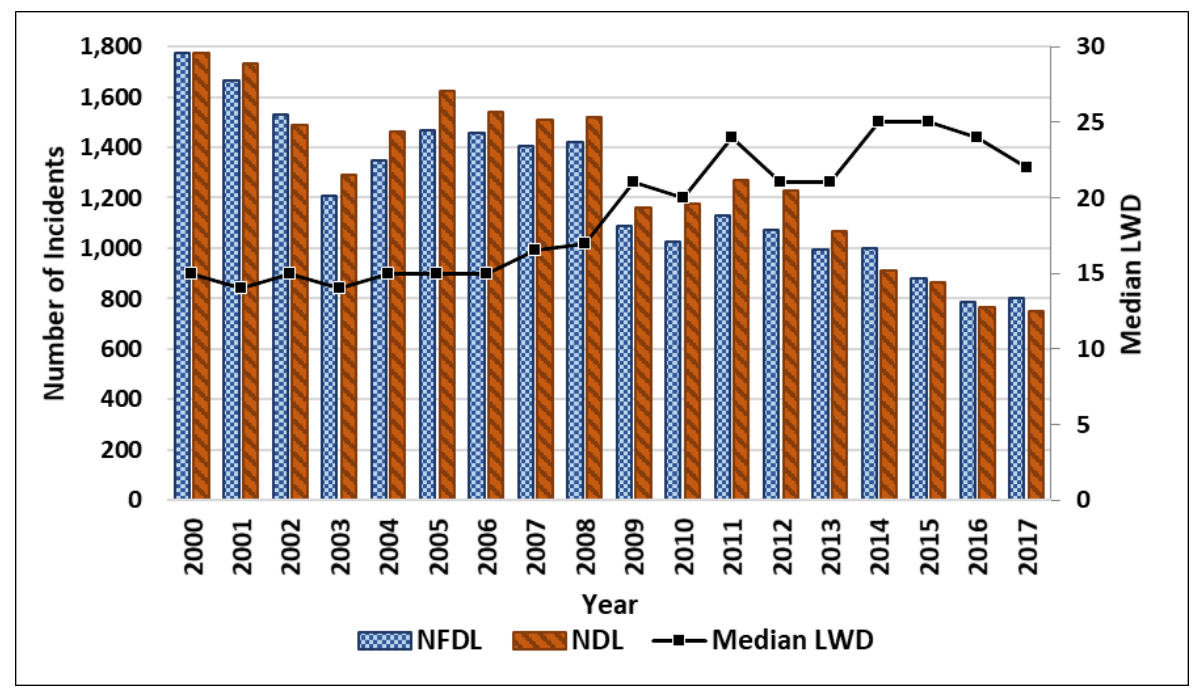

Figure 1: Number of NFDL, NDL incidents, and median LWD for the period from 2000 to 2017. NFDL and NDL incidents are plotted using bars and the primary axis to the left. Median LWD are plotted using line and the secondary axis to the right. 


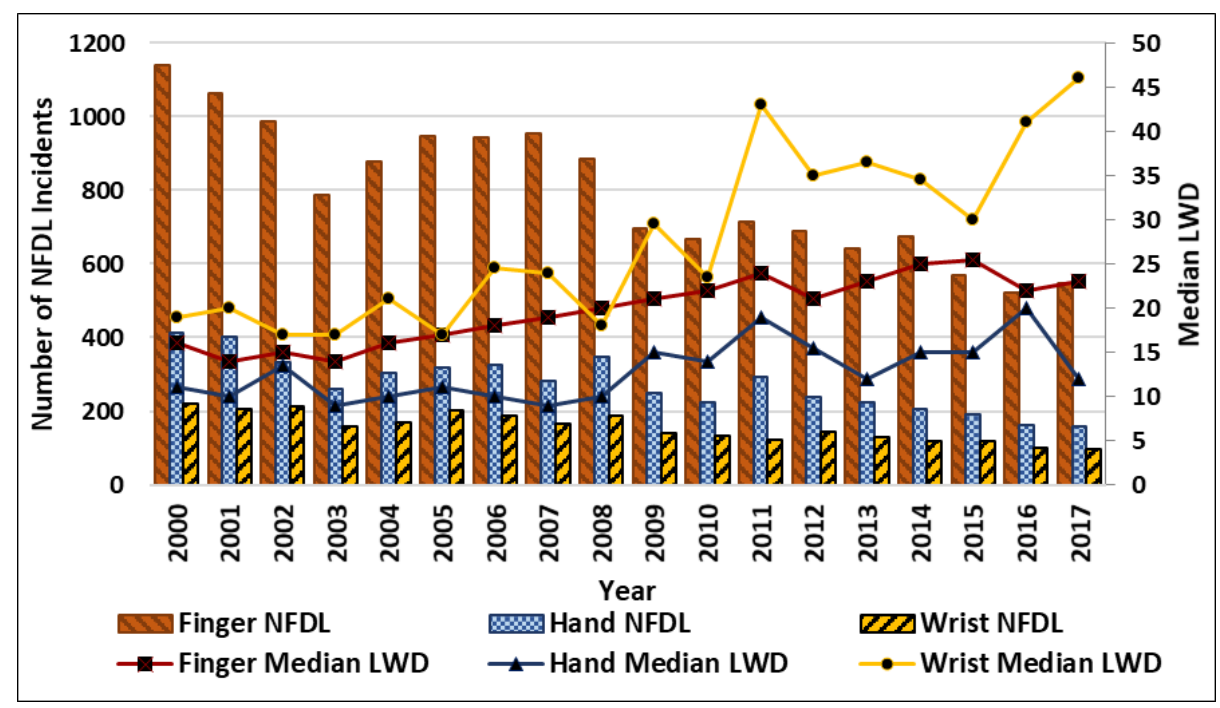

Figure 2: NFDL incidents and median LWD categorized by body parts for the period from 2000 to 2017. NFDL incidents are plotted using bars and the primary axis to the left. Median LWD are plotted using lines and the secondary axis to the right.

\subsubsection{Number of incidents within severity groups}

Figure 3 shows the total number of incidents and the resulted LWD for different severity groups. The number of incidents in the severity group [ $>60$ ] was 3,950 incidents $(8.7 \%$ of all incidents and $18 \%$ of NFDL incidents) which were responsible for nearly two thirds of the total LWD (613,873 LWD). When the severity groups with highest LWD were combined (i.e. [31-60] and [>60]), they included 7,936 incidents (18\% of all incidents and 36\% of NFDL incidents) which produced $84 \%$ of all LWD (790,727 LWD). The remaining 16\% LWD were distributed among the severity groups $[1-5],[6-10],[11-20]$, and $[21-30]$ as $2 \%, 3 \%, 5 \%$, and $6 \%$, respectively. More than half of all incidents were minor injuries and did not result in LWD (i.e. incidents in the severity group NDL). 


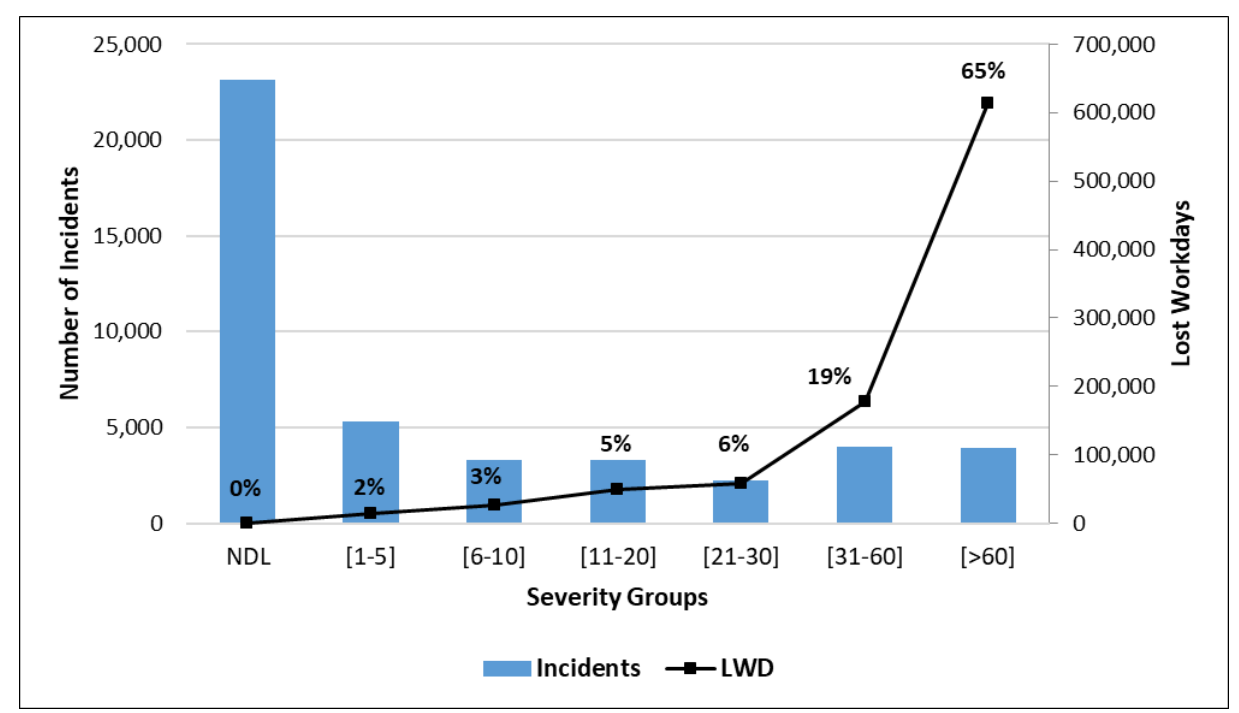

Figure 3: Total number of NFDL incidents and LWD for different severity groups. NFDL incidences are plotted using bars and the primary axis to the left. LWD are plotted using a line and the secondary axis to the right. LWD percentages are estimated with respect to the total LWD.

The yearly distributions of number of incidents for different severity groups is shown in Figure 4. Generally, all trends followed a decreasing pattern with some yearly variations. However, the amount of reduction in number of incidents from 2000 to 2017 was quite different between the severity groups. For instance, while the number of incidents decreased by $58 \%$ (from 1,774 to 751 incidents) in the severity group NDL, for the [>60] severity group it decreased by only $15 \%$ (from 203 to 172 incidents). Linear regression analysis of the data showed that the trends were statistically significant compared to the horizontal line for all severity groups $(p$-value $<0.05)$. A comparison of slope values showed that the groups $[1-5]$ and [>60] had the largest and smallest slope values, respectively (i.e. 0.38 and 0.06). The remaining groups can be arranged by decreasing slope as follows: [11-20], [6-10], NDL, [21-30], and [31$60]$. 


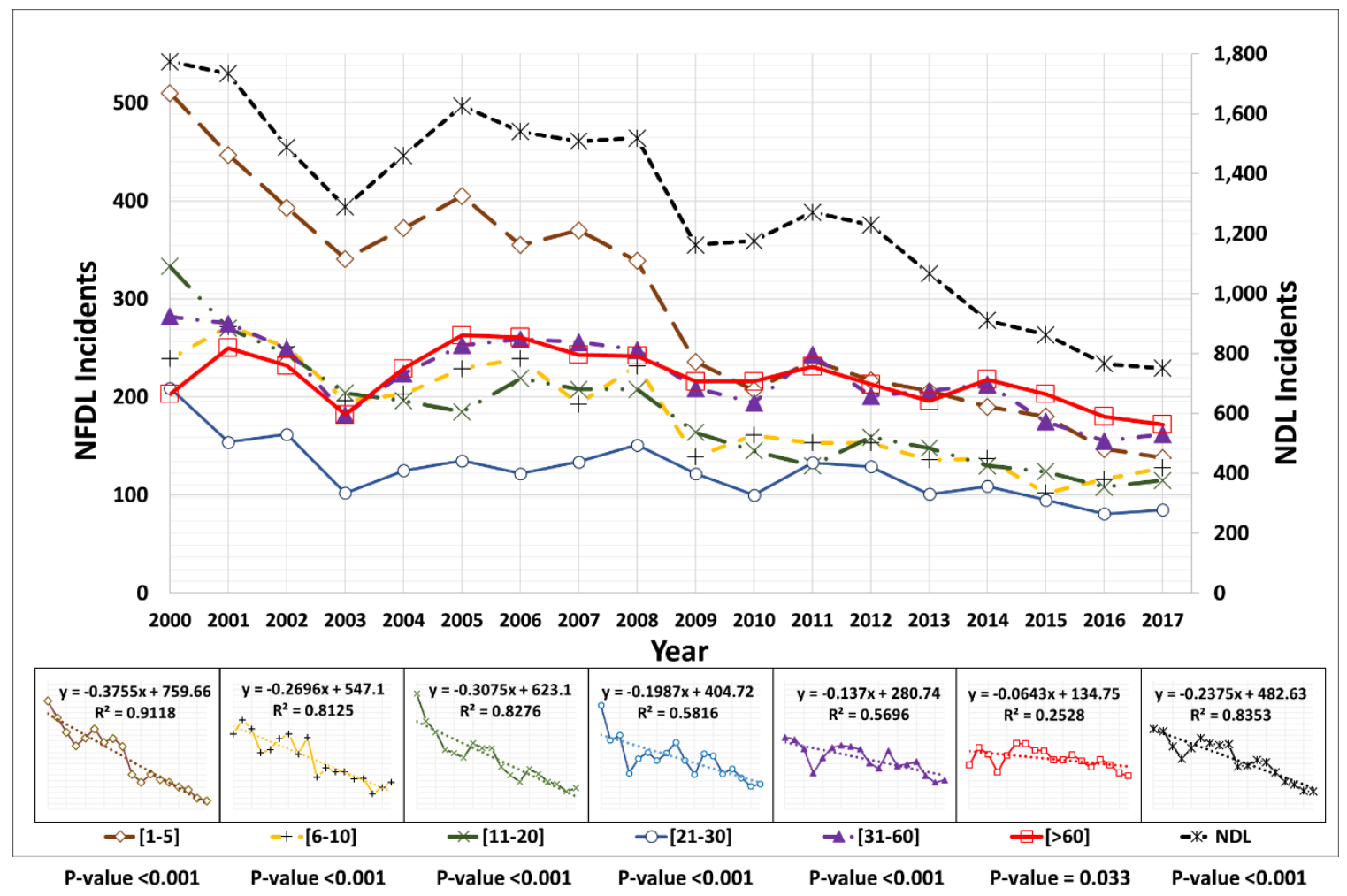

Figure 4: Yearly number of incidents for the severity groups and NDL. The secondary axis to the right is for NDL group and the primary axis to the left is for all other groups. Charts at the bottom were obtained from the normalized data and include regression equations and $\mathrm{R}^{2}$ values.

\subsubsection{Injury attributes association with severity groups}

\subsubsection{Accident type}

Association between accident type and severity group was found to be statistically significant $\left(\chi_{20}^{2}=622.73 ; P<.001\right)$. NFDL cases and the resulting LWD were higher for caught in and struck by compared to other accident types. Results of post-hoc comparisons (Table 2) showed that for caught in, observed numbers of incidents associated with the severity groups [21-30], [31-60], and [>60] were significantly higher than expected values. Median LWD for caught in was relatively high (21), and SI was the highest (24.6). For accident type struck by, observed number of incidents associated with the severity groups [31-60], and [>60] were significantly lower than expected values. However, the SI for struck by (11.6) was higher than the average SI for accident type (8.8). NFDL fall incidents associated with severity groups [31-60], and [>60] 
were significantly higher than expected values, and the median LWD was very high (30).

However, fall SI (5.5) was lower than average SI.

\subsubsection{Nature of injury}

A chi-squared association test showed that the association between nature of injury and severity group was statistically significant $\left(\chi_{45}^{2}=6,392.83 ; P<.001\right)$. Post-hoc analysis indicated that amputation incidents associated with severity groups [31-60], and [>60], and fracture incidents associated with severity groups [21-30], [31-60], and [>60] were significantly higher than expected values (Table 2). Fracture and amputation also had larger median LWD values (31 and 100, respectively) and larger SI values (17.7 and 36.3, respectively) compared to other injuries. Moreover, fracture and amputation incidents accounted for $61 \%$ of the total LWD. Cut/laceration NDL and NFDL incidents accounted for $66 \%$ and $31 \%$ of the total NDL and NFDL incidents, respectively. However, post-hoc analysis indicated that the number of incidents due to cut/laceration was significantly higher for low severity groups [1-5], [6-10], and [11-20]. Additionally, cut/laceration median LWD (10) and SI (4.1) were relatively low.

\subsubsection{Activity}

Significant association between activity and severity group was found by chi-square association tests $\left(\chi_{50}^{2}=524.62 ; P<.001\right)$. Post-hoc analysis showed that maintenance, operating equipment and roof bolter incidents in the severity group [>60] were significantly higher than expected values (Table 2). Maintenance had the highest SI value (12.1) and a relatively high median LWD (19). Operating equipment and roof bolter had high median LWD (23 and 26, respectively) and their SI (5.2 and 4.9, respectively) were larger than average SI for activity (4). The activities with the highest median LWD were climb scaffolds/ladders (38) and walking/running (28); however, they resulted in a very low number of NFDL incidents (123 and 
717, respectively), and they had low SI values (0.6 and 1.9, respectively). Moreover, the SI for other activities (5.3) was larger than average SI for activity (4) due to the large number of activity levels grouped into other activities (i.e. 53 levels).

\subsubsection{Experience}

Association between years of experience and severity group was found to be statistically significant $\left(\chi_{30}^{2}=259.75 ; P<.001\right)$. Numbers of incidents for workers with [0-5] years of experience associated with severity groups [31-60], and [>60] were significantly less than expected values. While median LWD for the [0-5] experience level was relatively low (15), SI value was the highest (19) compared to other experience levels. In general, the median LWD increased as years of experience increase with [>30] years of experience having the largest median LWD (i.e. 26). For [>30] years of experience, the number of incidents associated with severity group [>60] was significantly higher than the expected value.

\subsubsection{Subunit}

A significant association was found between subunit and severity group by chi-square association tests $\left(\chi_{20}^{2}=226.30 ; P<.001\right)$. Post-hoc analysis showed that for subunit underground, numbers of incidents associated with the severity groups [31-60], and [>60] were significantly higher than expected values (Table 3). Subunit underground had the highest median LWD (23) and SI (16.5). Mill operation and strip/quarry/open pit subunits SI values (12.2 and 11.7, respectively) were larger than mean SI for subunit (8.6).

\subsubsection{Injured finger}

The narrative text analysis resulted in identifying the name of injured fingers in $63 \%$ of NFDL finger incidents (Table 3). The distribution of the 9,036 total classified finger incidents 
between the levels of the injured finger variable (thumb, index, middle, ring, little, and multiple) were $26 \%, 20 \%, 17 \%, 12 \%, 15 \%$ and $10 \%$, respectively.

The chi-squared association test showed that the association between injured finger and severity group was statistically significant $\left(\chi_{50}^{2}=308.29 ; P<.001\right)$. For multiple and index, the number of incidents associated with severity group [>60] were significantly higher than expected. Also, multiple and index SI values (10.1 and 9.9, respectively) were larger than injured finger average SI (7.4). Multiple was associated with the highest median LWD (30), followed by index and middle (20). Thumb cases were associated with the highest cumulative LWD (105,544). For ring and little, numbers of incidents associated with severity group [31-60] were significantly higher than expected. The SI value for thumb (10.6) was the highest within injured finger levels. 
Table 2: Summary statistics and crosstabs for severity groups and accident type, nature of injury, and activity variables.

\begin{tabular}{|c|c|c|c|c|c|c|c|c|c|c|c|c|}
\hline \multirow[b]{2}{*}{ Variable } & \multicolumn{4}{|c|}{ Summary statistics } & \multicolumn{6}{|c|}{ Number of injuries within severity groups } & \multirow{3}{*}{$\begin{array}{c}\text { Severity } \\
\text { index } \\
(S I)\end{array}$} & \multirow{3}{*}{ Rank } \\
\hline & NDL & NFDL & LWD & $\begin{array}{c}\text { Median } \\
\text { LWD }\end{array}$ & {$[1-5]$} & {$[6-10]$} & [11-20] & {$[21-30]$} & {$[31-60]$} & {$[>60]$} & & \\
\hline Total & 23,135 & 22,044 & 938,467 & - & 5,290 & 3,279 & 3,290 & 2,249 & 3,986 & 3,950 & & \\
\hline \multicolumn{13}{|l|}{ Accident Type } \\
\hline Caught in & 8,023 & 9,279 & 485,835 & 21 & $1,898 \downarrow$ & $1,242 \downarrow$ & 1,398 & $1,036 \uparrow$ & $1,778 \uparrow$ & $1,927 \uparrow$ & 24.6 & 1 \\
\hline Struck by & 13,245 & 9,071 & 299759 & 13 & $2,513 \uparrow$ & $15,30 \uparrow$ & 1,354 & 871 & $1,524 \downarrow$ & $1,279 \downarrow$ & 11.6 & 2 \\
\hline Fall & 1,025 & 1,837 & 91,846 & 30 & $343 \downarrow$ & $178 \downarrow$ & $221 \downarrow$ & 189 & $424 \uparrow$ & $482 \uparrow$ & 5.5 & 3 \\
\hline Over exertion & 470 & 1,203 & 48,162 & 16 & 320 & 182 & 165 & 104 & 210 & 222 & 2.2 & 4 \\
\hline Other accident types & 372 & 654 & 12,865 & 9 & $2,16 \uparrow$ & $147 \uparrow$ & $152 \uparrow$ & 49 & $50 \downarrow$ & $40 \downarrow$ & 0.3 & 5 \\
\hline \multicolumn{13}{|l|}{ Nature of Injury } \\
\hline$\overline{\text { Amputation }}$ & 0 & 1,288 & 261,504 & 100 & $0 \downarrow$ & $0 \downarrow$ & $0 \downarrow$ & $0 \downarrow$ & $285 \uparrow$ & $1,003 \uparrow$ & 36.3 & 1 \\
\hline Fracture & 4,489 & 6,877 & 310,718 & 31 & $918 \downarrow$ & $583 \downarrow$ & 962 & $950 \uparrow$ & $1,949 \uparrow$ & $1,515 \uparrow$ & 17.7 & 2 \\
\hline Cut/laceration & 15,362 & 6,758 & 153,195 & 10 & $2,024 \uparrow$ & $1,548 \uparrow$ & $1,213 \uparrow$ & 640 & $806 \downarrow$ & $527 \downarrow$ & 4.1 & 3 \\
\hline Sprain/strain & 501 & 1,729 & 64,146 & 12 & $506 \uparrow$ & 289 & 254 & 147 & $242 \downarrow$ & 291 & 2.7 & 4 \\
\hline Crushing & 731 & 1,433 & 52,366 & 17 & 366 & 174 & 248 & 169 & 260 & 216 & 2.2 & 5 \\
\hline Other natures & 680 & 1,286 & 34,119 & 11 & $416 \uparrow$ & $235 \uparrow$ & $239 \uparrow$ & 112 & $145 \downarrow$ & $139 \downarrow$ & 1.1 & 6 \\
\hline Multiple injuries & 520 & 821 & 24,699 & 16 & 194 & 139 & 130 & 110 & 141 & $107 \downarrow$ & 0.9 & 7 \\
\hline Contusion/bruise & 688 & 1,652 & 29,820 & 6 & $812 \uparrow$ & 290 & 214 & $100 \downarrow$ & $126 \downarrow$ & $110 \downarrow$ & 0.6 & 8 \\
\hline Dislocation & 129 & 132 & 6,143 & 25.5 & 31 & 8 & 18 & 16 & 25 & 34 & 0.4 & 9 \\
\hline Joint inflammation & 35 & 68 & 1,757 & 9 & 23 & 13 & 12 & 5 & 7 & 8 & 0.1 & 10 \\
\hline \multicolumn{13}{|l|}{ Activity } \\
\hline Maintenance & 4,864 & 4,870 & 242,307 & 19 & $1,059 \downarrow$ & 709 & 733 & 508 & 840 & $1,021 \uparrow$ & 12.1 & 1 \\
\hline Handling material & 4,966 & 4,348 & 167,131 & 17 & 1,080 & 673 & 638 & 444 & 791 & 722 & 7.2 & 2 \\
\hline Other activities & 2,615 & 2,705 & 116,003 & 17 & 684 & 388 & 427 & 247 & 462 & 497 & 5.3 & 3 \\
\hline Operating equipment & 1,049 & 1,562 & 95,742 & 23 & 324 & 189 & 223 & 156 & 303 & $367 \uparrow$ & 5.2 & 4 \\
\hline Roof bolter & 1,247 & 1,894 & 91,231 & 26 & $371 \downarrow$ & $195 \downarrow$ & 278 & 210 & $431 \uparrow$ & $409 \uparrow$ & 4.9 & 5 \\
\hline Hand tools (not powered) & 5,974 & 3,506 & 99,920 & 11 & $1,022 \uparrow$ & $646 \uparrow$ & 554 & 360 & $539 \downarrow$ & $385 \downarrow$ & 3.3 & 6 \\
\hline Walking/running & 526 & 717 & 34,667 & 28 & 135 & 90 & 76 & 84 & $172 \uparrow$ & 160 & 1.9 & 7 \\
\hline Getting on/off equipment & 490 & 623 & 31,644 & 23 & 150 & 64 & 74 & 62 & 130 & 143 & 1.7 & 8 \\
\hline Hand tools (powered) & 766 & 787 & 26,658 & 16 & 197 & 128 & 123 & 77 & 154 & 108 & 1.0 & 9 \\
\hline Handling coal/rock/ore & 580 & 909 & 25,276 & 11 & 253 & $189 \uparrow$ & 143 & 88 & 140 & $96 \downarrow$ & 0.8 & 10 \\
\hline Climb scaffolds/ladders & 58 & 123 & 7,888 & 38 & 15 & 8 & 21 & 13 & 24 & $42 \uparrow$ & 0.6 & 11 \\
\hline
\end{tabular}

Up-arrow ( $\uparrow$ ) indicates that the observed value is significantly higher than the expected value. Down-arrow $(\downarrow)$ indicates that the observed value is significantly lower than the expected value. SI values greater than the mean SI within each variable are bolded. 
Table 3: Summary statistics and crosstabs for severity groups and experience, subunit, and injured finger variables.

\begin{tabular}{|c|c|c|c|c|c|c|c|c|c|c|c|c|}
\hline \multirow[b]{2}{*}{ Variable } & \multicolumn{4}{|c|}{ Summary statistics } & \multicolumn{6}{|c|}{ Number of injuries within severity groups } & \multirow{2}{*}{$\begin{array}{c}\text { Severity } \\
\text { index } \\
(S I)\end{array}$} & \multirow[b]{2}{*}{ Rank } \\
\hline & NDL & NFDL & LWD & $\begin{array}{l}\text { Median } \\
\text { LWD }\end{array}$ & {$[1-5]$} & {$[6-10]$} & [11-20] & {$[21-30]$} & {$[31-60]$} & {$[>60]$} & & \\
\hline \multicolumn{13}{|l|}{ Experience } \\
\hline$[0-5]$ & 10,864 & 11,828 & 460,066 & 15 & $3,164 \uparrow$ & $1,855 \uparrow$ & 1,791 & 1,194 & $1,960 \downarrow$ & $1,864 \downarrow$ & 19.0 & 1 \\
\hline$[6-10]$ & 3,712 & 3,424 & 153,902 & 19 & 790 & 472 & 518 & 360 & 612 & 672 & 7.4 & 2 \\
\hline [11-15] & 2,200 & 1,889 & 90,383 & 19 & 415 & 293 & 267 & 165 & 361 & 388 & 4.5 & 3 \\
\hline [16-20] & 1,654 & 1,397 & 63,713 & 21 & 289 & 210 & 183 & 149 & 291 & 275 & 3.1 & 4 \\
\hline [21-25] & 1,575 & 1,220 & 61,282 & 23 & $237 \downarrow$ & 147 & 192 & 132 & 261 & 251 & 3.1 & 5 \\
\hline [26-30] & 1,606 & 1,195 & 56,116 & 23 & $228 \downarrow$ & 149 & 177 & 133 & $267 \uparrow$ & 241 & 2.9 & 7 \\
\hline$[>30]$ & 1,524 & 1,091 & 53,005 & 26 & $167 \downarrow$ & 153 & 162 & 116 & 234 & $259 \uparrow$ & 3.0 & 6 \\
\hline \multicolumn{13}{|l|}{ Subunit } \\
\hline$\overline{\text { Underground }}$ & 8,160 & 7,137 & 325,756 & 23 & $1,549 \downarrow$ & $840 \downarrow$ & 1,021 & 771 & $1,490 \uparrow$ & $1,466 \uparrow$ & 16.5 & 1 \\
\hline Mill operation & 6,364 & 6,655 & 280,334 & 16 & 1,552 & $1,126 \uparrow$ & 1,036 & 688 & 1,127 & 1,126 & 12.2 & 2 \\
\hline Strip/Quarry/Open pit & 7,012 & 7,111 & 280,694 & 14 & $1,916 \uparrow$ & $1,140 \uparrow$ & 1,059 & 676 & $1,175 \downarrow$ & $1,145 \downarrow$ & 11.7 & 3 \\
\hline Surface at underground & 1,054 & 612 & 30,425 & 20 & 138 & 77 & 93 & 64 & 114 & 126 & 1.5 & 4 \\
\hline Other subunits & 545 & 529 & 21,258 & 14 & 135 & 96 & 81 & 50 & 80 & 87 & 0.9 & 5 \\
\hline Total (injured fingers) & - & 9,036 & 387,598 & - & 1,958 & 1,279 & 1,370 & 1,014 & 1,726 & 1,689 & - & - \\
\hline \multicolumn{13}{|l|}{ Injured finger } \\
\hline Thumb & - & 2,343 & 105,544 & 18 & $581 \uparrow$ & 325 & 363 & 260 & 434 & $380 \downarrow$ & 10.6 & 1 \\
\hline Multiple & - & 901 & 64,851 & 30 & $130 \downarrow$ & 102 & 114 & 108 & 165 & $282 \uparrow$ & 10.1 & 2 \\
\hline Index & - & 1,851 & 81,022 & 20 & 389 & 269 & 293 & 196 & 279 & $425 \uparrow$ & 9.9 & 3 \\
\hline Middle & - & 1,498 & 54,282 & 20 & 317 & 236 & 219 & 177 & $247 \downarrow$ & 302 & 6.2 & 4 \\
\hline Little & - & 1,344 & 44,389 & 19 & 309 & 198 & 197 & 132 & $333 \uparrow$ & $175 \downarrow$ & 4.2 & 5 \\
\hline Ring & - & 1,099 & 37,510 & 19 & 232 & 149 & 184 & 141 & $268 \uparrow$ & $125 \downarrow$ & 3.4 & 6 \\
\hline
\end{tabular}

Up-arrow ( $\uparrow$ ) indicates that the observed value is significantly higher than the expected value. Down-arrow ( $\downarrow)$ indicates that the observed value is significantly lower than the expected value. SI values greater than the mean SI within each variable are bolded. 


\subsubsection{Source of injury}

Source of injury was evaluated for NFDL injuries associated with activities which have the highest injury severity: maintenance, roof bolter, and operating equipment. Figure 5 illustrates the major sources of injury for maintenance activity. Metal parts (e.g. pipe, wire, and nails), metal covers and guards, and belt conveyors attributed to the largest proportions of maintenance NFDL injuries and LWD. Major sources of injury for roof bolter activity were categorized based on the main tasks they perform (Figure 6). For drilling tasks, drill steel, caving rock, coal, ore, or waste, and underground mining machines were the major sources of injury. The latter two were also identified as major sources of injury for inserting bolts, tramming, and other not elsewhere classified (NEC) tasks. Also, roof bolt was the major source of injury for the task inserting bolt. Major sources of injury for operating equipment activity are shown in Figure 7. The two major sources of injury were mine jeep, kersey and jitney tractors, and machines such as welder, bonder, lathe, and drill press. The remaining major sources contributed to approximately the same number of NFDL and LWD (Figure 7).

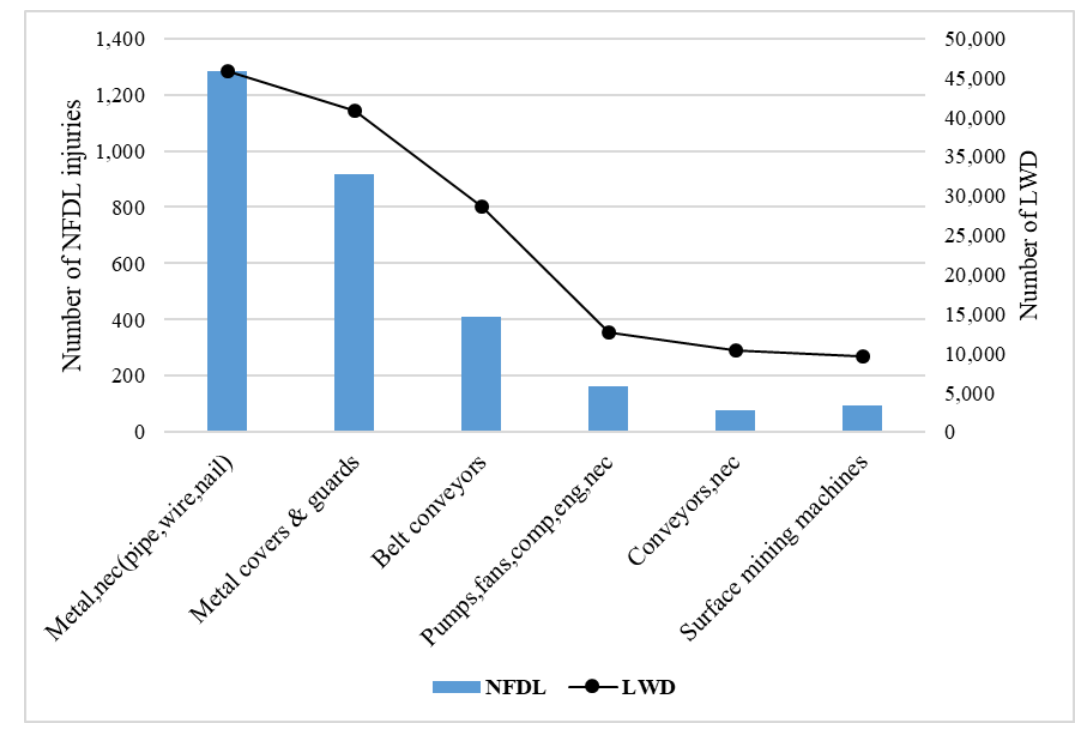

Figure 5: Number of NFDL injuries and LWD categorized by source of injuries that resulted in most injuries and LWD within maintenance activity. Note that only the main sources are included (69\% of maintenance NFDL injuries). 


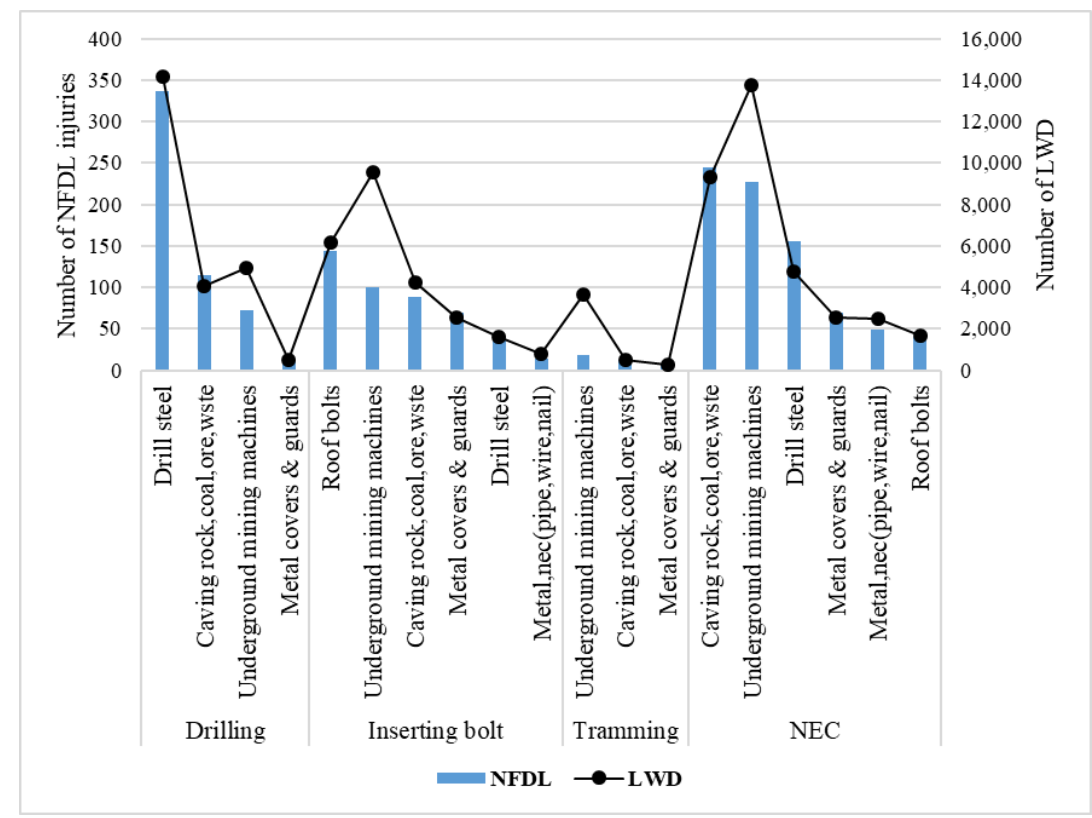

Figure 6: Number of NFDL injuries and LWD categorized by source of injuries that resulted in most injuries and LWD within roof bolter activities. NEC stands for "not elsewhere classified" and includes all injuries not in the main three categories. Note that only the main sources are included (95\% of roof bolter NFDL injuries).

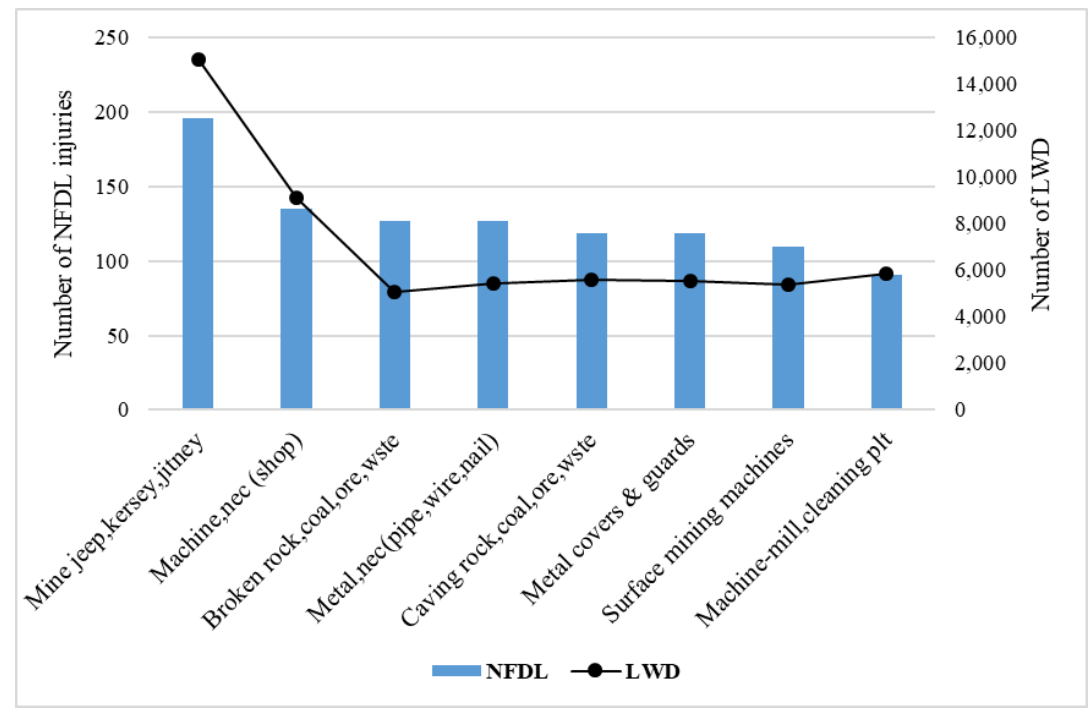

Figure 7: Number of NFDL injuries and LWD categorized by source of injuries that resulted in most injuries and LWD within operating equipment activity. Note that only the main sources are included (66\% of operating equipment NFDL injuries). 


\subsection{Discussion}

The main objective of this study was to investigate hand injuries in the mining industry, identifying causes and work characteristics which contribute to severe finger, hand, and wrist injuries. Our analysis provides basic information on how the number of incidences and severity of hand injuries in the mining industry evolved over the last 18 years. We also analyzed the data to understand relationship between different severity groups and various factors or work characteristics that influence injury causation. While the relationships were analyzed using a chisquared association test, we conducted additional supplemental analysis using adjusted standardized residuals and SI computations. The former investigated cellwise differences within a certain level and the latter considered column proportions within a severity group and LWD (i.e. global measure). Our analysis revealed several interesting trends.

The finger injuries accounted for the majority of the reported incidents (i.e. $69 \%$ ). The median LWD for wrist injuries (24) was higher than finger injuries (19) but finger injuries resulted in the highest cumulative LWD (66\%), which can be attributed to the large number of finger injuries. A similar trend regarding the finger injuries compared to wrist and hand injuries was also observed among emergency department visits (Hill et al., 1998; Jin et al., 2010; Larsen et al., 2004).

The overall number of hand injuries decreased from 2000 to 2017. This is consistent with the findings of other studies that showed an improvement in reducing number of mining injuries (Groves et al., 2007; Nasarwanji et al., 2018; Poplin et al., 2008). Over the same period, injury severity measured using median LWD increased. Reduction in the number of injuries was much smaller for the two high severity groups, [31-60] and [>60], compared to the other groups. Severe hand injuries could lead to an extended period of LWD, which was associated with nearly 
$75 \%$ of the total cost of hand and wrist injuries (De Putter et al., 2016). Aside from creating an economic burden for sufferers, severe hand injuries cause a notable impact on patients' quality of life. Kovacs et al. (2011) explored the effects of severe hand injuries on quality of life in 118 patients, reporting that patients with severe injuries had significantly lower satisfaction with their life and health, higher levels of depression and anxiety, and higher levels of body dysmorphic disorder.

Among the various accident types, struck by and caught in attributed to the majority of LWD ( 84\%). Pollard et al. (2014) investigated maintenance and repair injuries in the US mining industry from 2002 to 2011, and reported that struck by and caught in accident types resulted in a substantial number of injuries and LWD. They also reported that the fingers were the most affected body part from these injuries (at 20\%). Mital, Pennathur, \& Kansal (2000) examined nonfatal occupational injuries in upper extremities reported in the US in 1995. It was found that struck by and caught in accidents were the primary causes of finger injuries (i.e. 58\% and 34\% of finger injuries, respectively). Lind (2008) assessed industrial maintenance in Finland, and reported that fall and caught in accidents were among the leading causes of severe non-fatal injuries. Although fall incidents were associated with injuries with more lost workdays, fall SI was very low (5.5). Struck by accidents in the current study were primarily associated with less severe injuries and accounted for $32 \%$ of the total LWD with a relatively high SI (11.6), which can be attributed to the large number of NFDL injuries caused by struck by accidents (41\%).

The sum of fracture and amputation incidents resulted in $61 \%$ of the total LWD and were found to be significantly associated with severe injury groups ([31-60], and [>60]). Amputation injuries in the present study accounted for only $5.8 \%$ of NFDL injuries; however, they resulted in nearly $28 \%$ of the total LWD, which could explain its large median LWD and SI (i.e. 100 and 
36.3, respectively). This could also be attributed to the use of scheduled charges in LWD calculation. Several previous studies reported that fracture injuries are considered as one of the most common hand injuries among mine workers and in various other work environments (Nowrouzi-Kia et al. 2018; Lind 2008; Chung, Spilson, and Arbor 2001). Pollard et al. (2014) found that, after back and shoulder strains, fractures and lacerations of the hand resulted in the greatest number of LWD. Cut/laceration in the current study had a very low SI (4.1) indicating that cut/laceration NFDL injuries were associated with low number of LWD. However, they resulted in $16.3 \%$ of the total LWD, which could be due to the large number of NFDL cut/laceration incidents (i.e. $31 \%$ ).

Maintenance activity was also significantly associated with injuries resulting in a large number of LWD. Occupational safety literature often considers maintenance as a high-risk operation (Pollard et al., 2014; Reardon et al., 2014), which could be attributed to several risk factors, such as task-exceptional conditions (e.g. time of day), time pressure, and working at a running process (Lind, 2008). A previous analysis of maintenance and repair injuries in the US mining industry found that an average of 20 amputated fingers, 180 fractured hands and fingers, and 455 hand and finger lacerations was reported annually (Pollard et al., 2014). Adding such findings to the association of amputation and fracture with severe injuries as demonstrated by the current study further illustrates the association between maintenance and severe injuries. Since maintenance activity often involves dynamic and non-repetitive tasks, it is difficult to identify specific injury causing tasks. Our source of injury analysis indicates that metal parts such as pipe, wire, and nails pose an elevated risk of injury within the maintenance activity and tend to cause caught in (49\%) or struck by (47\%) accidents. Belt conveyors were also found to be a major source of injury with high risks of caught in accidents (78\%). 
Operating equipment and roof bolter were also significantly associated with an elevated injury severity. Groves et al. (2007) reported that from 1995-2004, operating a roof bolter was associated with the second highest number of NFDL and NDL incidents within the machinery category. The main tasks of roof bolter activity involve drilling holes into an unsupported roof, inserting roof bolts to prevent roof collapse, and tramming. Drilling and inserting bolts tasks, in the present study, resulted into the highest numbers of LWD compared to the other tasks (i.e. 24,265 and 25,637 respectively). For drilling and inserting bolts tasks, drill steel and roof bolts caused large proportions of the NFDL injuries, respectively. Also, drill steel resulted in $28 \%$ of all roof bolter NFDL injuries and 23\% of the resulted LWD within roof bolter activity. A brief evaluation of the narrative text was performed on a random small sample of drill steel NFDL injuries. This analysis showed that a common risk of drill steel was falling of roof and impacting worker hand. Another observation was that drill steel tends to get stuck in roof, bend or break because of excessive pressure, and then spring back and struck the worker. Drill steel also could present pinch-point risks when connected to the chuck or when two drill steels are connected. The sources of injury: caving rock, coal, ore, or waste, and underground mining machines were common across all roof bolter tasks.

A common source of injury across all three activities was metal covers and guards. MSHA regulations require all mines to prevent direct contact with hazardous moving machine parts by utilizing protection guards. However, metal covers and guards presented additional safety hazards accounting for nearly $16 \%$ of the total NFDL injuries (3,550 injuries) and $11 \%$ of the total resulted LWD (98,648 LWD). A possible explanation of such hazards could be attributed to the poorly designed protection guards which poses a significant materials handling risk (Pollard et al., 2014). Future studies could perform extensive and systematic evaluation of the narrative 
text to identify circumstances of the incidents and specific hazard sources within the hazardous activities.

The number of injuries for workers with [0-5] years of experience was significantly associated with low severity. However, because of the large number of NFDL injuries among workers in this group (54\%), their injuries resulted in nearly half of the total LWD (49\%) and they have higher SI (19) than the other experience levels. Other studies have shown similar findings; a majority of incidents involve workers with less than 5 years of experience (Groves et al., 2007; Lee et al., 1993; Weston et al., 2016). Injury severity was found to be higher for experienced workers. More experience is generally linked with older age. Previous studies have reported higher risks of injuries with elevated severity among the older workers (Laflamme \& Blank, 1996; Sammarco et al., 2016; Weston et al., 2016).

Median lost workdays for underground and surface at underground injuries were relatively higher than the other subunit levels, indicating an increased severity for injuries in these two subunits. Previous studies reviewed several articles regarding injuries in the US and global mining industries, and reported that underground mining is one of the main predictors of NFDL injuries and is one of the most hazardous environments (B. Nowrouzi-Kia et al., 2017; Behdin Nowrouzi-Kia et al., 2018; Poplin et al., 2008). The elevated injury risk and severity of underground subunit can be attributed to several factors, such as the use of heavy machinery (e.g. roof bolting machine), absence of natural light, and undesirable air temperature, humidity, and noise (Paul, 2009). Relatively high SI values for mill operation and strip/quarry/open pit subunits (12.2 and 11.7, respectively) indicated increased numbers of NFDL injuries associated with severe injury groups. 
With respect to locations of finger injuries, results of the narrative text analysis showed that thumb, index, and middle fingers were the most frequently injured fingers. A similar observation was reported in the literature (Jin et al., 2010; Sorock et al., 2004). While the thumb was the most injured finger in our analysis, Jin et al. (2010) and Sorock et al. (2004) identified index as the most frequent injured finger. Hill et al. (1998) reported that thumb, index, and little fingers were injured more often compared to other fingers. Davasaksan et al. (2012) investigated occupational hand injuries treated at hospitals between 1992 and 2005, finding that index and middle fingers were the most frequently injured fingers. These differences could be attributed to the different sampling methodologies, as other studies analyzed hospital and emergency department data. Multiple and index finger injuries were significantly higher than expected values under high severity groups, had the highest values of median LWD, and high SI values. The thumb had the highest SI value which is similar to the finding of Jin et al. (2010),

Hand injury prevention is often approached through engineering and administrative controls, hazard awareness, and the use of personal protective equipment. Mining workers are usually required to wear metacarpal gloves to provide impact protection for the hands and fingers. A previous study by Sorock et al. (2004) estimated the amount of protection provided by gloves and reported that the use of protective gloves could prevent laceration and puncture injuries. Although the use of gloves did not protect against crush or fracture injuries, the authors estimated a reduction of injury risk to $60-70 \%$. The choice of protective glove must depend on the nature of the task to be performed and the level of needed performance as gloves often are associated with reduced dexterity and increased muscle activity (Dianat et al., 2012). A gloves selection standard has been published and updated during the past few decades, and includes mechanical protection against cuts, punctures, and abrasion, chemical protection, heat and flame 
protection, and cold protection (ANSI/ISEA 105-2016). However, gloves impact protection was not standardized until recently (i.e. ANSI/ISEA 138-2019), which could explain the increased injury severity for impact related injuries (e.g. fracture). In other words, because of the lack of an impact resistance standard, it is possible that workers have not been using the proper metacarpal gloves for the needed impact protection. Future work evaluating gloves impact resistance and developing a protection index could be useful for choosing proper gloves for different tasks. This issue is the main focus of Aim \#2 of this project (chapters 3 and 4). Additionally, as more protection provided by gloves often compromises dexterity and comfort, optimization of glove designs with more protection provided for more vulnerable fingers could contribute to risk reduction.

\subsection{Conclusion}

Results of this study identify several factors that contribute to severe hand injuries. These factors can be grouped into different injury severities by combining results of post-hoc comparison based on the adjusted standardized residual method and the SI computations: (1) Factors with significantly higher than expected values under high severity groups and high SI can be classified into "very high severity" category. The caught in accident type, fracture and amputation injuries, maintenance, operating equipment, and roof bolter activities, underground subunit, and multiple and index fingers are the "very high severity" factors. (2) Factors with not significantly higher than expected values under high severity groups and high SI can be classified into "high severity" category. The struck by accident type, handling material activity, less experienced workers ( $<5$ years), mill operation and strip/quarry/open pit subunits, and the thumb are the "high severity" factors. (3) Factors with significantly higher than expected under high severity groups and low SI can be classified into "moderate severity" category. The fall 
accident type, walking/running and climb scaffolds/ladders activities, more experienced workers (> 25 years), and the ring and little fingers are "moderate severity" factors.

Targeting these factors in future prevention and intervention programs may result in reduction in number and severity of hand injuries. This research also suggests that future efforts focused on hand injuries in the mining industry should be directed towards monitoring and reducing severity in addition to attempting to reduce incident rates. This is a challenging proposition, as each task presents its own issues and requires careful study and thorough planning. For example, reducing exposure to caught in accidents, from an engineering control perspective, could be partially achieved by adding proper handles and guards to equipment. However, in order to do that properly, an extensive investigation of the causes and effects of caught in accidents is required. 


\section{Chapter 3. Specific Aim \#1: Development and testing of impact protection measurement methods for the metacarpal gloves}

\subsection{Introduction}

The main goals of our preliminary study presented in Chapter 2 were to fill the knowledge gap in the literature regarding hand safety of mine workers, and to evaluate the levels and prevalence of severe hand injuries related to impact accidents. The results of the preliminary study showed that over the last two decades, 84\% of the total LWD (790,727 LWD) associated to wrist, hand, and finger injuries was caused by $18 \%$ of the total reported wrist, hand, and finger injuries with a median LWD greater than 30 days. For these severe injuries, the struck by accidents, fractures, and amputation injuries were prevalent and were linked to the lack of adequate hand protection against impact accidents. A previous assessment of clinically acute hand injuries estimated that the reduction in injury risk due to gloves use was in the range of 60 to $70 \%$ (Sorock et al., 2004). Due to the high occurrence of severe occupational hand injury in the mining industry (Alessa et al., 2020), mine workers often were metacarpal gloves as a PPE.

Metacarpal gloves are typically comprised of fabric layers (synthetic or natural materials) with external reinforcements of thermoplastic rubber (TPR). The TPR is the molded material placed on the dorsal side of the glove, which is mainly intended to provide impact protection. TPR reinforcements are typically placed along the dorsal portion of the fingers and thumb, on top of the knuckles, and the dorsal metacarpal region of the hand. Some glove models only include thick pads of fabric layers placed on the dorsal and palmar areas of the hand.

The variety of glove designs and constructions makes it difficult for the end-users to select the most suitable glove for a given task. The technical literature shows very few attempts that 
substantiate evaluation and/or selection of metacarpal gloves against impact hazards. In one of the studies, impact forces required to induce fracture of different bones of the hand were assessed using cadaveric hands (Loshek, 2015). The range of age of the cadaveric hands was between 76 and 98 years of age, with an average age of 87 years, which can limit the validity of the reported results. Moreover, the same study shows significant variability in the results, and only a minimal amount of detail regarding the methods used in the study are provided. The same study measured the reduction in hand impact force as a measure of glove performance against impact and compared the performance of different gloves against no-glove testing. Still, only a part of the results obtained is publicly available. In a more recent study, Carpanen et al. (2019) created risk curves to evaluate the probability of injury of unprotected metacarpophalangeal (MCP) and proximal interphalangeal (PIP) joints of 21 cadaveric hands subjected to blunt impacts. While their study provides valuable information on the range of forces necessary to induce fracture of MCP and PIP joints, it only includes data for unprotected hands.

\subsubsection{Objectives}

Based on the findings of our preliminary study, as well as to address the knowledge gap in the literature about glove impact resistance evaluation, this study aims to perform an experimental quantification of the impact protection performance of selected commercially available metacarpal gloves. The specific objectives of this part of the study were: (1) to develop a data set and improve our knowledge on how the human hand structures react to blunt impact by measuring forces during controlled impacts on unprotected and protected hands; and (2) to generate basic comparison matrix for a selected metacarpal glove based on different indicators, including maximum reaction to the impact force, number of fractures, and an index indicative of 
the level of protection offered by the gloves. The collected data and knowledge are essential for the development and validation of a surrogate hand which is discussed in Chapter 4.

\subsection{Methods}

The experimental approach selected for this research included the use of thirteen fresh-frozen cadaveric hand specimens for controlled impact tests. The specimens came from male donors with ages in the range of 38 to 66 years and an average age of 53 years (Standard Deviation (SD) $=11$ years), which better represent the targeted population compared to previous similar studies (Carpanen et al., 2019; Loshek, 2015). The specimens consisted of five pairs, right and left, and three single right hands, for a total of 13 specimens. These specimens were provided by the West Virginia University (WVU) Human Gift Registry in coordination with the WVU Department of Orthopaedics and the WVU Department of Mechanical Engineering. The study followed biosafety and handling procedures approved by the WVU Institutional Biosafety Committee. In preparation for the tests, the specimens were thawed at laboratory room temperature $\left(\sim 23^{\circ} \mathrm{C}\right)$ for 24 hours before testing. Prior to the tests, all specimens were inspected to ensure the absence of trauma, anatomical irregularities, or evident damage that could distort the measurements.

\subsubsection{Experimental design}

Controlled impact tests were performed on each proximal interphalangeal (PIP) joint (including thumb interphalangeal joint), on each metacarpophalangeal (MCP) joint, and the middle point of each metacarpal bone, for a total of 15 impacts per hand specimen. The test was designed to evaluate the performance of metacarpal gloves against impact forces. Thus, six specimens were impacted with two types of metacarpal gloves on ( 3 specimens per glove type), and seven specimens were impacted without gloves (unprotected tests) and used as a baseline for comparison. For each pair of hands, one specimen was tested with a glove on ("with-glove" 
condition), and the other was tested without a glove ("no-glove" condition). Two of the three single specimens were tested without a glove, and one was tested with a glove. The single specimen tested with a glove was paired to the average of the two single specimens tested without a glove.

The two types of metacarpal gloves selected for this study were considered to have different levels of protection based on their designs, as well as the position and quantity of thermoplastic rubber (TPR). The first glove (G1) includes TPR reinforcements only on fingers region and foam padding on MCP joints and back of hand (metacarpal bones) region. The second glove (G2) includes TPR reinforcements on fingers, MCP joints, and the metacarpals region, as shown in Figure 8(a) and (b), respectively. Detailed specifications of the selected gloves are presented and discussed in Chapter 4 (Section 4.3.2). The sizes of the gloves G1 and G2 used in the test were XL and L, depending on the ability to insert the glove on tested hands. Detailed information about hands dimensions and the gloves used are presented in Table 8.

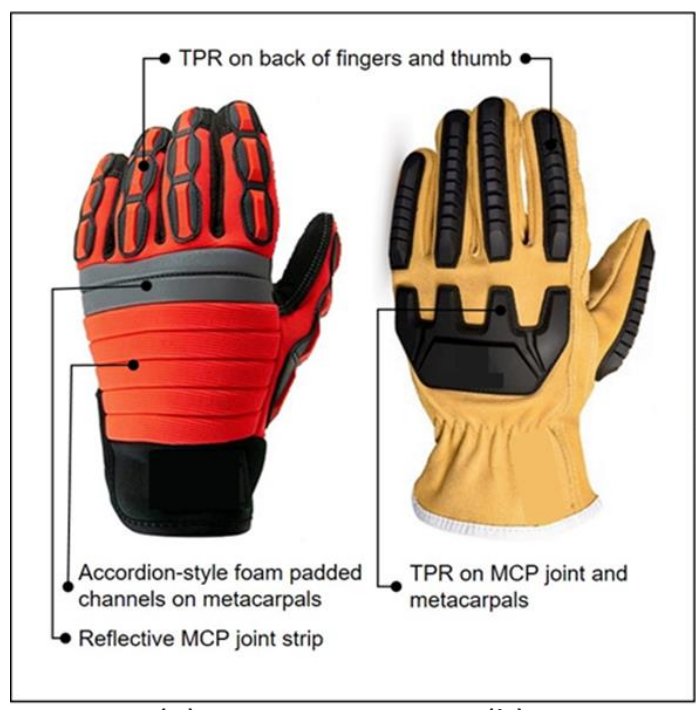

(a)

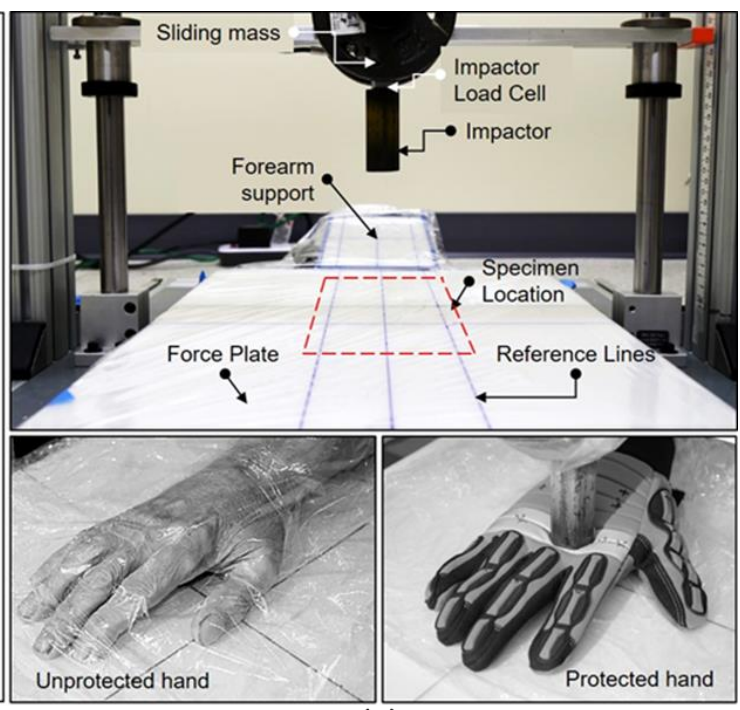

(c)

Figure 8: Metacarpal gloves and test setup: (a) Glove G1; (b) Glove G2; (c) Components of impact testing setup (top) and testing specimens (bottom) 


\subsubsection{Testing set-up description}

A dual column (guillotine type) testing apparatus with a vertical sliding mass attached to linear bearings was used for this experiment, as shown in Figure 8(c). This machine was also used in the experiments conducted by Sosa et al. (2019). A hexagonal-shaped impactor with a flat striking face, $25 \mathrm{~mm}$ outer diameter, and a nominal impact area of $\sim 406 \mathrm{~mm}^{2}$ was mounted below the sliding mass. The cross-sectional shape was derived from a drill steel bar typically used in mining roof bolting activities, which have been reported by Sammarco et al. (2016) to frequently fall off and impact mine-workers' hands. The same finding was also suggested by the work performed during preliminary study (Alessa et al., 2020). The sliding mass was connected to an electromagnetic release mechanism which allowed for controlled and safe release. A fixture (forearm support) with an adjustable elevation and inclination angle was used to position the specimens with the hand and forearm in a resting posture, as shown in Figure 8(c).

The vertical impact reaction force (z-axis) was measured using a force plate (Bertec FP4060NC-1000, Bertec Corporation, Columbus, OH) placed on the surface underneath the impactor. A load cell (Loadstar RSB3, Loadstar Sensors, Fremont, CA) was mounted in between the sliding mass, and the impactor to measure the impact reaction force transferred back to the impactor (i.e., through the impacted specimen and glove). The rated load of the used force plate and load cell on the vertical direction (z-axis) was $5 \mathrm{kN}$. The weight of the sliding mass, including the weight of the attached load cell and impactor, was $5.1 \mathrm{~kg}$. A string potentiometer displacement sensor (Loadstar ISP-125, Loadstar Sensors, Fremont, CA) was mounted to the frame of the testing apparatus and connected to the sliding mass to monitor the impactor displacement. Impact force and displacement data were recorded at $1 \mathrm{kHz}$ using the "MotionMonitor" data acquisition software (MotionMonitor, Innovative Sports Training, Chicago, IL). 
At the time of testing, the closest standard available for impact testing was the motorcycleglove impact-protection standard (BS EN 13594:2015), which suggests conducting impact tests with an impact energy of $5 \mathrm{~J}$. A preliminary pilot study was conducted with different energy levels and impact forces were compared to force values reported in the literature (Carpanen et al., 2019; Loshek, 2015). From this pilot study, it was found that $5 \mathrm{~J}$ was not sufficient to produce fractures consistently, which was also reported by Carpanen et al. (2019). In order to evaluate the level of protection provided by the selected metacarpal gloves against severe injuries (i.e. fractures), the energy was set to a level that would likely produce a bone injury as a result of the impact. Thus, the drop height was set to $0.2 \mathrm{~m}$, which in combination with the sliding mass, would put the impact energy at a theoretical level of $10 \mathrm{~J}$. It is worth noting that the impact testing apparatus could cause some energy loss due to the machine friction. The friction of the testing apparatus was estimated at $13 \%(\mathrm{SD}=3 \%)$ following the procedure described in a previous study (J. Z. Wu et al., 2019). This value is comparable to their estimation of the energy loss of an impact testing machine with a mass of $5 \mathrm{~kg}$ used in a construction helmets standardized test.

\subsubsection{Testing Procedure}

Specimens were first inspected to detect external anomalies and then radiographed to assess the initial condition and verify the absence of previous fractures in the bone structure of the specimens. Posteroanterior, lateral, and oblique views of the specimens were captured with a

portable X-ray machine (DRX-Revolution Mobile X-ray System, Carestream Health, Rochester, NY). For the condition of "with glove" testing, the hand specimen was inserted into one of the selected gloves (G1 or G2), and another set of radiographic images was taken to ensure proper fitting. Impact locations were then marked onto the glove dorsal region to maintain test 
consistency across all specimens. Next, the specimen was placed on the force plate with the hand in resting and nearly flat position and with the forearm resting and secured to the adjustable fixture. The impact testing was divided into three consecutive stages, starting with five impacts on each digit's PIP (including thumb IP) joint, followed by five impacts on each MCP joint, and completed with five impacts on the middle point of each metacarpal bone. After each set of impacts, posteroanterior, lateral, and oblique radiographic images were obtained to quantify the number of fractures in each region independently. All radiographic images were captured by a trained technician.

\subsubsection{Data processing}

\subsubsection{Impact forces}

The peak reaction forces were captured for each impact at two points: (a) the peak reaction force (PRF) underneath the impact zone of the specimen measured by the force plate; and (b) the peak reaction force transferred back through the specimen (TPRF) measured by the load cell mounted on the impactor. The difference between PRF and TPRF was used to measure the force reduction (FR) percentage as an indirect measure of energy dissipation provided by gloves. The FR value may be influenced by the individual stiffness of the hand specimens. However, this influence was assumed to be substantially reduced since FR values are calculated as an average of all specimens tested using the same glove. Additionally, vertical displacement data was used to derive the velocity, which in turn was used to calculate the effective kinetic energy right before the impact.

For each glove type, the average PRF of each region was compared to that of the specimens with no-glove. The stiffness and dampening properties of the gloves were different due to the differences in their designs and materials used, and therefore it was anticipated that gloves would 
produce differences in the PRF values. Thus, change in PRF between no-glove and with-glove conditions was used as an indicator of the level of protection provided by gloves. Furthermore, an Impact Protection Index (IPI) adopted from the work of Sosa et al. (2019) was used to quantify the amount of protection for each glove. This index combines impact reaction forces obtained from various locations for pairs of protected and unprotected hand specimens and amount of FR. IPI value between 0 and 100 can be calculated using Equation 1.

$$
I P I_{G}=\left[1-\left(\sum_{p=1}^{3} w_{p} \times\left(\frac{P R F_{p(G)}}{P R F_{p(\text { no glove })}}-F R_{p(G)}\right)\right)\right] \times 100
$$

Where:

G represents the type of metacarpal glove (1 represent G1, and 2 represent G2);

$\mathrm{p}$ is the impacted position ( 1 for PIP joint, 2 for MCP joint, and 3 for metacarpals);

$\mathrm{w}_{\mathrm{p}}$ is the weight assigned for an impacted position (p) and obtained from accident analysis (preliminary study);

$\mathrm{PRF}_{\mathrm{p}}(\mathrm{G})$ is the average $\mathrm{PRF}$ at position $\mathrm{p}$ for glove $\mathrm{G}$;

$\mathrm{PRF}_{\mathrm{p}}$ (no-glove) is the average PRF at position $\mathrm{p}$ for the condition of "no-glove";

$\mathrm{FR}_{\mathrm{p}}(\mathrm{G})$ is the average $\mathrm{FR}$ at position $\mathrm{p}$ for glove $\mathrm{G}$.

The weighting factors $\left(\mathrm{w}_{\mathrm{p}}\right)$ were derived from historical hand injury data reported by MSHA for the period from 2000 to 2017 which is analyzed within the preliminary study (Alessa et al., 2020). Only finger and hand data were considered (wrist injuries were not included in the $\mathrm{w}_{\mathrm{p}}$ calculation). The data showed that hand and finger injuries resulted in 187,485 (23\%) and $616,696(77 \%)$ lost workdays, respectively. Lost workdays are often used as a measure of injury 
severity. Since the data did not classify injuries at the knuckles area (MCP) independently and included them within the hand injury level, it was assumed that each of MCP and metacarpal bones accounted for half the injuries in this category. This assumption was only made for the calculation of $\mathrm{w}_{\mathrm{p}}$ values. Thus, $\mathrm{w}$ for PIP joints was set to 0.77 and $\mathrm{w}$ for MCP joints, and metacarpal bones was set to 0.115 (i.e., $0.23 / 2$ ).

\subsubsection{Bone strength evaluation}

Bone strength is often evaluated using bone mineral density (BMD) (Plato \& Norris, 1980), which is known to decline with age (Boonen et al., 2009). BMD is usually determined using dual-energy x-ray absorptiometry (DXA), which is a technique that was not available for this study. Instead, we adopted a simple approach of estimating the tubular BMD from standard radiograph images by measuring cortical thickness of the second and third metacarpals (Ashok Kumar et al., 2018; Barnett \& Nordin, 1960; Fox et al., 1995; Ives \& Brickley, 2004).

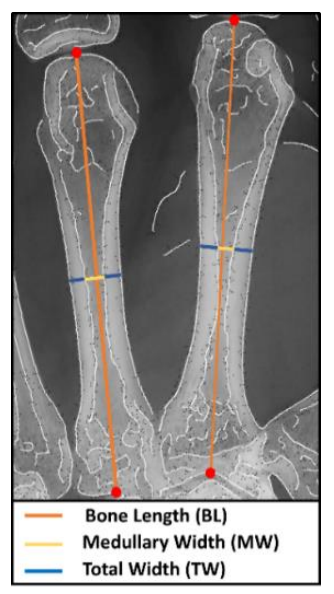

Figure 9: Illustration of edges created by Canny filter and measurement positions.

Previous metacarpal radiogrammetry studies have often performed the measurements manually using a caliper or digital ruler using Dicom viewers. However, manual measurements are prone to observers' error and can be time-consuming (Ives \& Brickley, 2004). A few attempts have been made in the past to automate the measurement of metacarpal cortical bone 
thickness using different edge detection methods (e.g., Canny, Prewitt, and Sobel) (Khalid et al., 2010; Raheja, 2008; Shubhangi et al., 2012). In this work, to minimize observer error while executing systematic measurements, the Canny edge detection method (Canny, 1986) was used to perform the metacarpal cortical thickness measurements. Khalid et al. (2010) showed that Canny filter results are comparable to manual measurements. A customized program was developed in Matlab-2019 (MathWorks, Natick, MA, USA), in which an x-ray image is imported into the program, and the operator only marks two edge points (head and base) on a cropped image of a metacarpal bone (Figure 9). The program automatically measures the bone length (BL), medullary width (MW), and total width (TW) of the selected bone. In order to confirm the measurements obtained with the Canny filter, a manual method using a ruler tool (Dicom viewer) with an accuracy of $0.01 \mathrm{~mm}$ was used as a secondary method. Detailed procedures of the manual measurements are explained in the work of Ives \& Brickley (2004). BL is the distance between the upper margin of the metacarpal head and the notch at the base of the bone (Figure 9). MW and TW were measured at the midpoint of BL. Next, averages of MW and TW of second and third metacarpal bones were calculated. Average cortical thickness (CT) was defined as the difference between averages of TW and MW (CT = TW-MW). Cortical Index (CI) was then calculated for each specimen as the proportion of average $\mathrm{CT}$ from average $\mathrm{TW}(\mathrm{CI}=$ (TW-MW)/TW) (Ashok Kumar et al., 2018; Glencross \& Agarwal, 2011). Higher values of CI indicate higher bone density. As a reference, values of CI falling below 0.43 were linked to abnormal bones (Barnett \& Nordin, 1960; Glencross \& Agarwal, 2011). All metacarpal cortical thickness measurements were performed on the posteroanterior X-ray images obtained from the specimens before the impact tests. Glencross \& Agarwal (2011) evaluated the difference between CI's of paired right and left second metacarpals of 12 pairs and reported a non- 
significant difference (less than $4.5 \%$ ). Thus, in the current study, pairs were initially assumed to be similar unless otherwise shown in the x-ray images or CI values.

\subsubsection{Fracture evaluation}

Two radiologists evaluated radiographic images to identify and quantify the number of fractures produced by the impacts. Impacts that resulted into fractures were considered injurious impacts. Overall numbers of injurious impacts in each region were normalized to the total number of impacts in that region. The resulting values were used as a protection measure to compare glove vs. no-glove conditions.

\subsubsection{Statistical analysis}

Prior to the statistical analysis, all assumptions of the analysis of variance (ANOVA) statistics were tested and verified (Montgomery, 2012) (see Appendix A for details). Two-way analysis of variance (ANOVA) was conducted to test the effect of Protection and Position on PRF values. The variable "Protection" was treated at two levels: no-glove and with-glove. The "with-glove" level combined data from specimens tested with gloves G1 and G2 together under one category. The "no-glove" level included all specimens tested without a glove. The variable "Position" was treated at three levels: PIP joint, MCP joint, and metacarpal bones. Additionally, student's t-test analysis was performed to compare the mean PRF values for different gloves and impact positions. Based on a study by Sosa et al. (2019) it was expected that the use of gloves can reduce PRF. Thus, a one-tailed t-test was performed to test the hypothesis that PRF from the with-glove level was significantly less than PRF from the no-glove level. A criterion $p$-value of $\leq$ 0.05 was used in all statistical analyses, which were performed in JMP Version 14 (SAS Institute Inc., Cary, NC). Descriptive analysis was used to compare number of fractures as explained in Section 3.2.4.3. 


\subsection{Results}

\subsubsection{Impact force evaluation}

A total of 191 controlled impacts were performed in the present study: 103, 43, and 45 impacts for the no-glove, glove G1, and glove G2 conditions, respectively. The results of ANOVA tests showed that the effects of the independent variables Protection (Table 4) and Position (Table 5) on the values of PRF were statistically significant $(\mathrm{P}<0.001)$ (See Appendix B for ANOVA tables). The interaction effect of the two independent variables was statistically not significant $(\mathrm{P}=0.447)$. Results summarized in Table 6 demonstrate the effect of Protection on the mean change of PRF across all Positions calculated using the student's t-test. At the PIP joints, MCP joints, and Metacarpal Positions, the use of glove resulted in statistically significant reductions in the PRF by $5.62 \%, 16.93 \%$, and $10.08 \%$, respectively $(\mathrm{P}=0.0022,0.0206$, and 0.0206 , respectively).

Table 4: Results of ANOVA test for the effect of Protection on PRF. Bold P-value indicates a statistically significant difference.

\begin{tabular}{|c|c|c|c|c|}
\hline Protection & No-Glove & With-Glove & $\begin{array}{c}\text { PRF Change } \\
{[\%]}\end{array}$ & $P$-value \\
\hline Average PRF (SD) [N] & $1,971(553)$ & $1,776(543)$ & 9.88 & 0.0120 \\
\hline
\end{tabular}

Table 5: Results of ANOVA test for the effect of Position on PRF. Bold P-value indicates a statistically significant difference.

\begin{tabular}{c|ccc|c}
\hline Position & PIP & MCP & Metacarpals & $P$-value \\
\hline Average PRF (SD) [N] & $2,415(431)$ & $1,661(359)$ & $1,563(423)$ & $<\mathbf{0 . 0 0 1}$ \\
\hline
\end{tabular}


Table 6: Summary statistics, Results of the students' t-test for the effect of Protection on PRF. Bold $\mathrm{P}$-value indicates a statistically significant difference.

\begin{tabular}{l|cc|c|c}
\hline \multirow{2}{*}{ Position } & \multicolumn{2}{|c|}{ Average PRF (SD) [N] } & PRF & \multirow{2}{*}{ P-value } \\
\cline { 2 - 3 } & No-Glove & With-Glove & Change [\%] & \\
\hline PIP & $2,481(443)$ & $2,341(411)$ & 5.62 & $\mathbf{0 . 0 2 9 1}$ \\
MCP & $1,799(359)$ & $1,495(285)$ & 16.93 & $\mathbf{0 . 0 0 2 2}$ \\
Metacarpals & $1,639(451)$ & $1,473(376)$ & 10.08 & $\mathbf{0 . 0 2 0 6}$ \\
\hline
\end{tabular}

Results summarized in Table 7 show the mean PRF, TPRF, calculated kinetic energy (KE), and the IPI for the with and no glove conditions. For G1 pairs, the average in the PRF between the "no-glove" and "with-glove" conditions for PIP joints, MCP joints, and Metacarpals Positions were $-3.0 \%, 10.0 \%$, and $12.6 \%$, respectively. On the other hand, average changes of PRF for the G2 pairs were: $13.7 \%$ for PIP joints, $23.4 \%$ for MCP joints, and $4.3 \%$ for Metacarpals. Only paired comparisons were carried out in the IPI analysis to reduce the error caused by specimens' age and condition differences. The values of IPI for the evaluated gloves were $10.3 \%$ for glove G1 and $23.5 \%$ for glove G2. The average KE for both protection levels, "no-glove" and "with-glove, was $7.8 \mathrm{~J}$.

Table 7: Summary of average PRF, TPRF, KE, and IPI for each glove type compared to their pairs of no-glove tests.

\begin{tabular}{|c|c|c|c|c|c|c|c|c|c|}
\hline \multirow{2}{*}{$\frac{0}{0}$} & \multirow[b]{2}{*}{ Position } & \multicolumn{3}{|c|}{ No-Glove (NG) } & \multicolumn{3}{|c|}{ With-Glove (G) } & \multirow{2}{*}{$\begin{array}{c}\mathrm{PRF}_{(\mathrm{NG})}- \\
\mathrm{PRF}_{(\mathrm{G})} \\
{[\%]}\end{array}$} & \multirow{2}{*}{$\begin{array}{l}\text { IPI } \\
{[\%]}\end{array}$} \\
\hline & & $\begin{array}{c}\text { PRF } \\
{[N]}\end{array}$ & $\begin{array}{c}\text { TPRF } \\
{[\mathrm{N}]}\end{array}$ & $\begin{array}{l}\mathrm{KE} \\
{[\mathrm{J}]}\end{array}$ & $\begin{array}{c}\text { PRF } \\
{[N]}\end{array}$ & $\begin{array}{c}\text { TPRF } \\
{[\mathrm{N}]}\end{array}$ & $\begin{array}{l}\mathrm{KE} \\
{[\mathrm{J}]}\end{array}$ & & \\
\hline \multirow{3}{*}{ G1 } & PIP & 2,270 & 2,008 & 7.8 & 2,337 & 2,081 & 7.8 & -3.0 & \multirow{3}{*}{10} \\
\hline & MCP & 1,676 & 1,515 & 7.7 & 1,509 & 1,417 & 7.9 & 10.0 & \\
\hline & Metacarpals & 1,551 & 1,392 & 7.4 & 1,356 & 1,257 & 7.7 & 12.6 & \\
\hline \multirow{3}{*}{$\mathrm{G} 2$} & PIP & 2,718 & 2,320 & 8.0 & 2,345 & 2,101 & 7.9 & 13.7 & \multirow{3}{*}{23} \\
\hline & $\mathrm{MCP}$ & 1,935 & 1,787 & 8.1 & 1,481 & 1,368 & 7.7 & 23.4 & \\
\hline & Metacarpals & 1,654 & 1,471 & 7.4 & 1,583 & 1,472 & 7.3 & 4.3 & \\
\hline
\end{tabular}




\subsubsection{Bone density evaluation}

TW and MW were measured using two methods, the Canny filter and manual measurements. Figure 10(a) shows that the Canny filter method slightly underestimated metacarpal TW and MW measurements compared to manual measurements; however, both methods provided comparable average values of CI, as shown in Figure 10(b). Thus, only measurements obtained using the Canny filter method were used to evaluate the CI, pair similarity, as summarized in Table 8. From this table, the average $\mathrm{CI}$ for all 13 specimens was $0.57(\mathrm{SD}=0.07)$. Initial assessment of pairs similarity showed a small difference between all pairs' CIs (i.e., $<10 \%$ ) except for specimen SP7, for which the right hand (SP7-NG-Right) had a CI $=0.45$, that compared to the left hand, $\mathrm{CI}=0.59$, produced a $24 \%$ difference in the CI, indicating a possible presence of an anomaly.

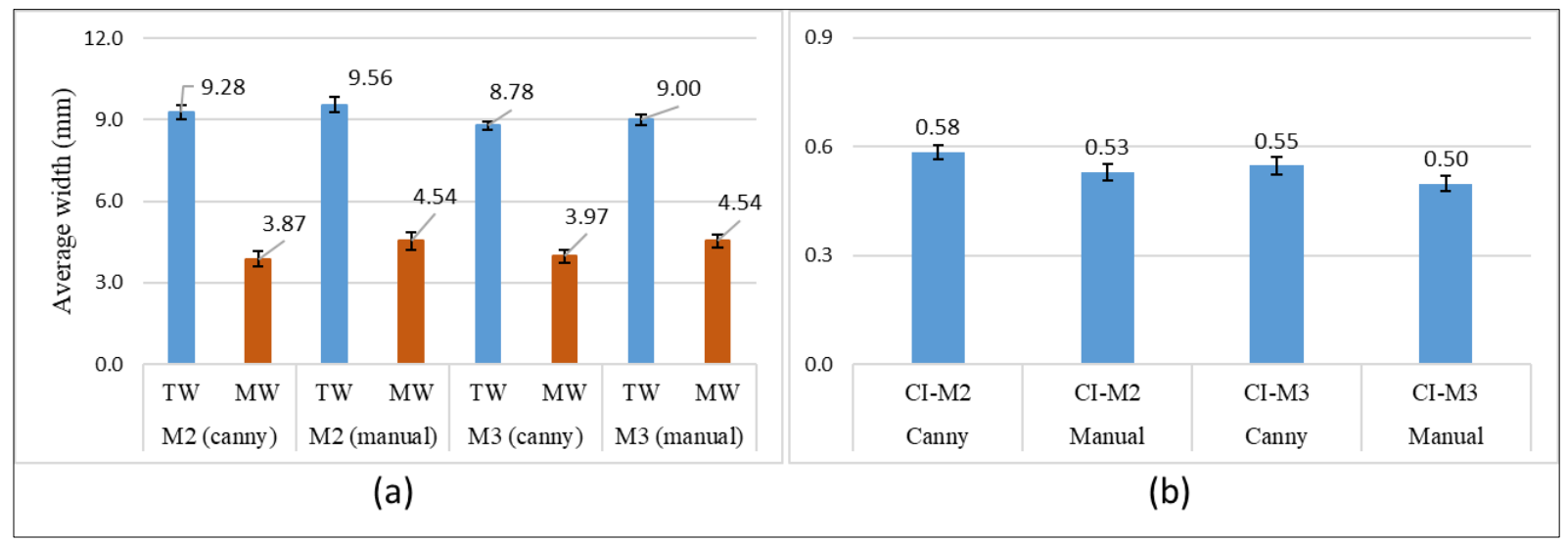

Figure 10: (a) Average total width (TW) and medullary width (MW); (b) Average cortical index (CI). Measurements made with the Canny filter and manual measurements for metacarpals M2 and M3. Bars represent standard error. 
Table 8: Tested specimens' information. Donor age, hand length \& breadth (measured from x-ray images), average values of second and third metacarpal TW, MW, CT, and CI for each specimen, and total number of fractures after impact.

\begin{tabular}{l|c|cc|c|ccc|c|c|c}
\hline \multicolumn{1}{c|}{ Specimen } & $\begin{array}{c}\text { Age } \\
\text { [years }]\end{array}$ & $\begin{array}{c}\text { Hand } \\
\text { length } \\
{[\mathrm{mm}]}\end{array}$ & $\begin{array}{c}\text { Hand } \\
\text { breadth } \\
{[\mathrm{mm}]}\end{array}$ & $\begin{array}{c}\text { Glove } \\
\text { size }\end{array}$ & $\begin{array}{c}\text { TW } \\
{[\mathrm{mm}]}\end{array}$ & $\begin{array}{c}\text { MW } \\
{[\mathrm{mm}]}\end{array}$ & $\begin{array}{c}\mathrm{CT} \\
{[\mathrm{mm}]}\end{array}$ & CI & $\begin{array}{c}\text { Pairs' CI } \\
\text { difference } \\
{[\%]}\end{array}$ & $\begin{array}{c}\text { Distribution } \\
\text { of fractures } \\
\mathrm{N}=108\end{array}$ \\
\hline $\begin{array}{l}\text { SP1-NG } \\
\text { (single) }\end{array}$ & 41 & 16.80 & 9.50 & - & 8.20 & 3.82 & 4.38 & 0.53 & - & 3 \\
\hline $\begin{array}{l}\text { SP2-NG } \\
\text { (single) }\end{array}$ & 38 & 20.30 & 9.30 & - & 9.17 & 4.03 & 5.14 & 0.56 & - & 4 \\
\hline SP3-G1- & 57 & 21.50 & 9.54 & $\mathrm{XL}$ & 9.52 & 3.41 & 6.12 & 0.64 & - & 6 \\
(single) & & & & & & & & & \\
\hline SP4-NG-Right & 61 & 19.73 & 9.47 & - & 7.92 & 2.85 & 5.07 & 0.64 & $2.2 \%$ & 8 \\
SP4-G1-Left & 61 & 19.94 & 9.42 & $\mathrm{XL}$ & 7.85 & 2.71 & 5.14 & 0.65 & \\
\hline SP5-NG-Left & 57 & 19.80 & 9.50 & - & 8.90 & 4.52 & 4.38 & 0.49 & $3.8 \%$ & 14 \\
SP5-G1-Right & 57 & 19.70 & 9.20 & $\mathrm{XL}$ & 9.14 & 4.46 & 4.67 & 0.51 & \\
\hline SP6-NG-Left & 66 & 19.25 & 9.10 & - & 9.45 & 4.33 & 5.12 & 0.54 & $8.8 \%$ & 10 \\
SP6-G2-Right & 66 & 19.20 & 8.20 & $\mathrm{~L}$ & 10.39 & 5.26 & 5.13 & 0.49 & 5 \\
\hline SP7-NG-Right & 57 & 19.80 & 9.20 & - & 9.87 & 5.42 & 4.45 & 0.45 & $24.1 \%$ & 28 \\
SP7-G2-Left & 57 & 19.70 & 9.20 & $\mathrm{~L}$ & 9.59 & 3.89 & 5.70 & 0.59 & \\
\hline SP8-NG-Left & 38 & 21.70 & 9.60 & - & 8.66 & 3.05 & 5.61 & 0.65 & $2.0 \%$ & 6 \\
SP8-G2-Right & 38 & 21.50 & 9.70 & $\mathrm{XL}$ & 8.76 & 3.20 & 5.56 & 0.63 & \\
\hline Average & 54 & 19.92 & 9.30 & - & 9.03 & 3.92 & 5.11 & 0.57 & 8.2 & 8.3 \\
SD & 11 & 1.26 & 0.38 & - & 0.76 & 0.87 & 0.54 & 0.07 & 0.09 & 6.8 \\
\hline
\end{tabular}

\subsubsection{Fracture evaluation}

The 191 controlled impacts produced a total of 108 fractures. The number of fractures per specimen is summarized in Table 8, while Figure 11(a) summarizes the distribution of fractures per Protection condition. From this graph, 68\% of the fractures were in no-glove condition, 20\% in glove G1 condition, and 12\% in glove G2 conditions. Also, Figure 11(b) summarizes the overall proportions of injurious impacts in relation to the total number of impacts at each impact position. For the no-glove condition, the proportions of fractures on PIP and MCP joints, and Metacarpal positions were 85\%, 57\%, and 71\%, respectively. Also from Figure 11(b), when all protection conditions are considered, the proportion of injurious impacts reduced to $60 \%, 34 \%$, and $24 \%$, for each impact position, for an overall $40 \%$ for all positions, which show that, as 
expected, the protection conditions (gloves G1 and G2) contributed to reducing the percentage of injurious impacts.

However, the reduction of injurious impacts was not the same for both types of gloves (Figure 11(c)). Considering all Positions, $71 \%$ of the impacts on specimens with no-glove were injurious, while $51 \%$ and $29 \%$ of the impacts on gloves G1 and G2 were injurious, respectively. Also, for glove G1, when evaluated by region of impact, $87 \%$ of the impacts on the PIP joints, $43 \%$ on MCP joints, and $21 \%$ on the Metacarpals, were injurious. Within glove G2, the percentages of injurious impacts on PIP and MCP joints, and Metacarpals were 33\%, 27\%, and $27 \%$, respectively.

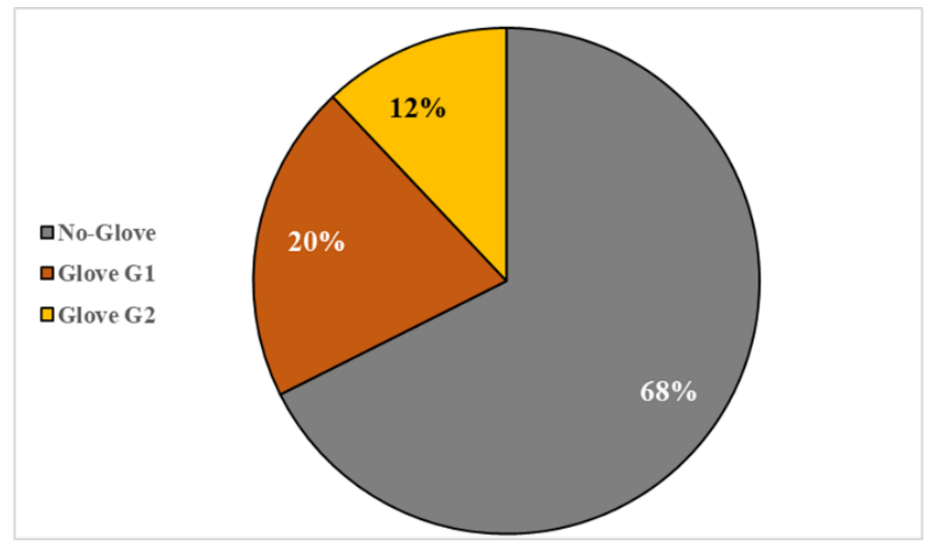

(a)

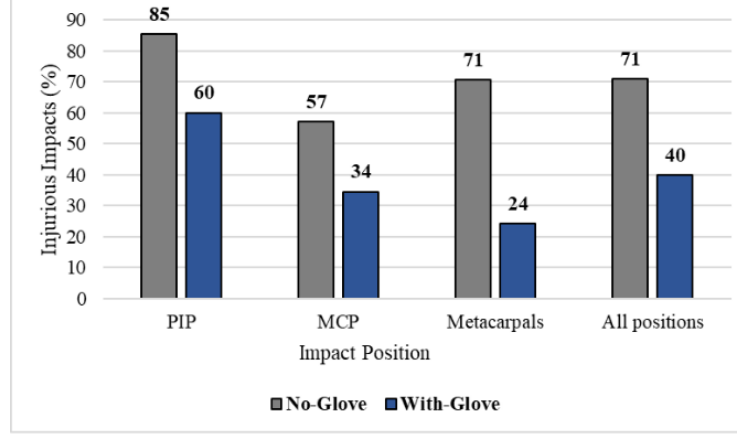

(b)

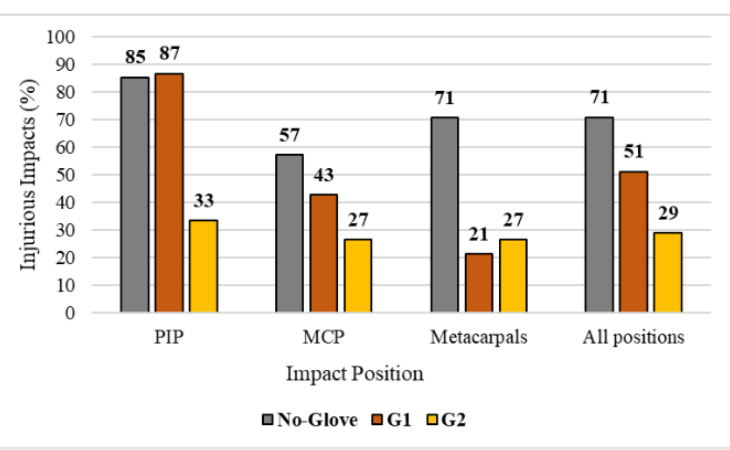

(c)

Figure 11: (a) Distribution of injurious impacts (Total fractures $, N_{F},=108$ ) for each Protection condition; (b) Percentage of impacts that resulted into a fracture (injurious impacts) per Position for all Protection conditions; (c) Percentage of impacts that resulted into a fracture (injurious impacts) for each Position and

Protection condition. For (b) and (c), percentages were calculated from the total number of impacts in each Position for each Protection condition. 


\subsection{Discussion}

A series of controlled impact tests were conducted on unprotected and protected human cadaveric hand specimens to quantify the level of reaction forces and the number of injurious impacts. Two types of metacarpal gloves were considered for the assessment by comparing their peak reaction forces (PRF) and the number of fractures for specimens with and without gloves.

All impact tests were carried out under the same testing conditions with a mass of $5.1 \mathrm{~kg}$, and a drop height of $0.2 \mathrm{~m}$ measured from the surface of the force plate. This combination would produce a nominal energy of $10 \mathrm{~J}$. However, the average kinetic energy calculated for all impact tests was $7.7 \mathrm{~J}$. The fluctuations of kinetic energy seen in Table 7 are attributed to the variability in specimens' depth at the location of the impacts, which reduced the drop distance to an average of $0.181 \mathrm{~m}$. Specimens are thinner at the PIP joints, and thicker at the metacarpals. Part of the energy loss is also attributed to the friction of the testing machine, which was estimated at $13 \%$ $(\mathrm{SD}=3 \%)$ following the procedure described in a previous study (J. Z. Wu et al., 2019). This value is comparable to their results for the $5 \mathrm{~kg}$ mass used to test the influence of testing machine friction on impact tests of construction helmets. Despite the energy loss, the measured reaction forces were sufficient to produce fractures and to capture the variations in performance for the different protection conditions and impact positions.

For the level of impact energy described above, and for the no-glove condition, the average PRF was 2,481 N, 1,799 N, and 1,639 N for the PIP and MCP joints, and Metacarpals, respectively (Table 6). A previous study obtained an average PRF of 3,673 N, 2,672 N and 2,957 $\mathrm{N}$ for the PIP and MCP joints, and Metacarpals, respectively (Loshek, 2015). Also, another study estimated that the forces for the 50\% injury risk in the MCP and PIP joints were 3,000 and 4,200 N, respectively (Carpanen et al., 2019). The values obtained in this study are, on average, about 
59\% and 67\% smaller than the two previous studies (Carpanen et al., 2019; Loshek, 2015).

These differences are attributed to the following factors: (a) number of impacts per location: in the current study each location (PIP and MCP joint and Metacarpal) was impacted only once. Previous similar studies (Carpanen et al., 2019; Loshek, 2015) produced more than one impact at some of the locations, at a higher energy level, if a fracture was not detected in the first attempt. The repeated impact of an apparently undamaged bone could have affected the reaction forces in the subsequent impacts; (b) bone condition of the specimens: the bone strength can be characterized by measuring the bone mineral density (BMD). The BMD measured in Loshek (2015) study ranged from $0.29 \mathrm{~g} / \mathrm{cm}^{2}$ to $0.35 \mathrm{~g} / \mathrm{cm}^{2}$ in four specimens and $0.52 \mathrm{~g} / \mathrm{cm}^{2}$ in two specimens, with no specific gender information. In this regard, a previous study (Lucas et al., 2008) showed that BMD measured in the forearm of men reduces with age (average of 0.56 $\mathrm{g} / \mathrm{cm}^{2}$ for the age group of 20-39 years to $0.49 \mathrm{~g} / \mathrm{cm}^{2}$ for the age group of 70 and older). Carpanen (2019) did not specify the gender of the specimens, nor report the BMD, and also acknowledged that the injury risk curves reported in their study might be overestimating the risk of injury in younger populations. In our study, all the test specimens were male, and the CI was used to assess bone condition before the impact tests. The CI values ranged from 0.45 to 0.65 , with an average of $0.57(\mathrm{SD}=0.07)$. The CI lowest value $(0.45)$ corresponded to one specimen that underwent 28 fractures at the different locations of impact, indicating the presence of an underlying bone anomaly. In this regard, previous reports indicated that CI values falling below 0.43 were linked to abnormal bones (Barnett \& Nordin, 1960; Glencross \& Agarwal, 2011).

The metacarpal gloves assessed in this study contributed to a statistically significant reduction of the PRF (i.e., $9.88 \%, \mathrm{P}=0.0120$, Table 4). This result indicates that, as expected, the gloves (either glove G1 or G2) dissipated some of the impact energy transferred to the hand. 
A more detailed analysis of average PRF corresponding to each glove type and their pairs of noglove tests (Table 7) showed that glove G2 dissipated the impact forces better than glove G1 in the PIP joints and the MCP joints (13.7\% and 23.4\%, respectively). However, comparing PRF changes in the Metacarpals between G1 and G2 results suggested that glove G2 dissipated less force (4.3\%) than glove G1 (12.6\%). It is speculated that this result is due to at least one specimen of the pair SP7 (which used glove G2) having an underlying bone anomaly that could have affected the force-carrying capacity of the hand.

Moreover, for specimens tested with glove G1, the average PRF in the PIP joints was slightly higher for with-glove condition $(2,337 \mathrm{~N})$ compared to the no-glove condition $(2,270 \mathrm{~N})$ (Table 7). Such findings indicate that while G1 caused reduction of the impact force in the MCP joints and Metacarpals (10.0\% and 12.6\%, respectively), it provided no force dissipation at the PIP joints (-3.0\%). This finding is consistent with the proportion of fractures shown in Figure 11(c). The chart of Figure 11(c) indicates that specimens with no-glove condition displayed nearly the same proportion of fractures observed on the specimens wearing glove G1, and thus, did not contribute to reducing the number of fractures observed in the PIP joints. These results are considered unusual since the design of glove G1 includes TPR reinforcements in the fingers, including the PIP joints, and only foam padding on MCP joints and Metacarpals, as shown in Figure 8 (a). A possible justification for these results could be attributed to the stiffness or hardness of the TPR reinforcements used in glove G1, which may not be suitable to dissipate the impact energy, as well as to the variability of the hand specimens used for the tests. A limitation of this study is the reduced number of specimens available for the tests of each glove type. Further tests would be needed under different levels of impact energy to establish more accurate levels of glove performance. 
A reduction in transferred impact energy could hypothetically result in injury risk reduction, which was assessed by the ratio between the number of fractures and the total number of impacts for all Protection conditions. In this regard, Figure 11(b) shows that this ratio was $71 \%$ and $40 \%$ for the no-glove and with-glove conditions, respectively. Thus, the use of metacarpal gloves can be associated with preventing nearly $44 \%$ of the fractures. Moreover, the percent reduction in the number of fractures with the use of gloves was 30\% for PIP joints, $40 \%$ for MCP joints, and $66 \%$ for Metacarpals. It is important to note that amount of risk reduction could change under different impact energy levels. A previous assessment of clinical acute hand injuries suggested that although the use of gloves did not protect against crush or fracture injuries, the estimated injury risk reduction due to gloves use was in the range of 60 to $70 \%$ (Sorock et al., 2004).

The IPI calculated in the current study were smaller than a previous impact test study that was performed using a semi-flexible surrogate hand (Sosa et al., 2019). IPI values of $40 \%$ and $51 \%$ for gloves G1 and G2, respectively were reported by Sosa et al. (2019), whereas in the current study IPI values for the same gloves were $10 \%$ and 23\%, respectively. These results suggest that the combined stiffness of the hand, and the materials of the glove may affect the PRF, which in turn, affects the IPI values. Considering hand stiffness in the test or utilizing the IPI as an indicator which require testing glove and no-glove conditions could allow for more precise quantification of protection provided by a glove. Such knowledge could aid safety professionals for better identification and selection of suitable gloves for the different activities of the users. Furthermore, the data generated from this cadaveric study could be useful for developing surrogate hand with biomechanical properties similar to human hands. Surrogate hands could provide cost effective methods for generating comparison matrix for the metacarpal gloves. 


\section{Chapter 4. Specific Aim \#2: Development of surrogate hand for performance evaluation of metacarpal gloves}

\subsection{Introduction}

Gloves impact testing has been performed in the past to evaluate the reduction of peak impact force (Loshek, 2015), which can be linked to bone fractures and severe bruising in the human hand (Carpanen et al., 2019). The use of living human subjects for impact force measurements is not feasible due to the risk of severe injuries caused by the impact forces. For such type of testing, cadaveric specimens were used in the literature (Carpanen et al., 2019; Schuurman \& Kauer, 2002; Yoganandan et al., 2016). A few other relevant examples include the testing of personal protective equipment such as head helmet (Hardy et al., 2007; Trotta et al., 2018) and wrist brace (Greenwald et al., 2010).

The use of cadaveric specimens, despite their usefulness for valuable measurements and acquiring realistic data, has several limitations. Cadaveric specimens are often difficult to acquire, limiting the sample size of the study. Other limitations include the variability caused by the age of the specimens, typically obtained from older donors, as well as the previous health conditions of the specimens. These limitations can further limit the sample size desired for a targeted population and may affect the interpretation and accuracy of study findings.

A possible approach to tackle such limitations is to develop and use a simulator (surrogate) to the human body part, for example, dummies used for car crash tests (Byrnes et al., 2002) and the headforms used for helmet evaluations (Bonin et al., 2017; Trotta et al., 2018). Previously, Hummel et al. (2011) used a hand simulator to measure the thermal protection provided by gloves. In their study, a hand simulator was instrumented with thermal sensors to measure the 
heat transmission through the gloves. Human body parts simulators were also commonly used in medical residency training programs (Lim et al., 2016; Y. Y. Wu et al., 2016). For instance, Wu et al. (2018) used a high-fidelity tactile hand simulator for the training of percutaneous pinning. The hand simulator was developed using bones produced by additive manufacturing (3D printing) technique and soft tissues formed by casting of ballistic gel material.

3D printing technologies have gained tremendous attractiveness in recent years with the introduction of low-cost printers into the market. It has been increasingly utilized for several medical applications such as the development of tailored prosthodontic implants and joints replacements (Ackland et al., 2018; Sun \& Zhang, 2012), cranial reconstructions (Jardini et al., 2014), and to manufacture models of human body parts for medical education (Lim et al., 2016). Advanced multi-material 3D printing techniques can produce surrogate parts with almost identical mechanical and stiffness properties. Such advanced techniques involving printing multi-materials may not be cost effective for experimental testing with large sample requirements.

\subsubsection{Objectives}

This study is aimed at development of scale of performance for commonly used metacarpal gloves. Controlled impact tests (same as in Aim \#1) were used to test the performance of metacarpal gloves. To overcome the various limitations encountered during Aim \#1 (small sample size, age variability, difficulty of testing and high cost of specimens), surrogate hands were designed, manufactured and used in the impact testing. The study objectives of this part of the study were: (1) To design and manufacture a surrogate hand that mimics the hard tissue (bone structure) and the surrounding soft tissue of the human hand to deliver impact testing results comparable to the results obtained with the cadaveric specimens. A 3D printing and 
casting process were used to develop and manufacture a surrogate hand to closely match the biomechanical proprieties of a human hand; (2) To evaluate the level of protection of metacarpal gloves under impact loads using the newly developed surrogate hand.

\subsubsection{Approach}

A dual material model was selected for creating a synthetic surrogate hand. The hand bone structure is created by an additive manufacturing technique (3D printing) and all the soft tissues surrounding the bone structure are represented by medical-grade synthetic gel. The proportions and size of the surrogate hand corresponded to the $50^{\text {th }}$ percentile of the population. Digital models of the human hand were used to create, and 3D print the bone structure, as well as to develop the gel casting process for the soft tissues. The experimental data obtained from Aim \#1 was used to fine tune the design of surrogate hand. The resulted surrogate hand was then used to test the impact performance of three types of commercially available metacarpal gloves typically used in the mining industry.

\subsection{Objective 1: Design and evaluation of a surrogate hand}

This part includes two main sections: (1) detailed explanation of the steps followed in the design and manufacturing processes of surrogate hand, and (2) testing and evaluation conducted to improve the design of surrogate hand.

\subsubsection{Design methodology}

A unique combination of several small and intricate bones, soft tissues and ligaments provide human hand its complex and sophisticated structure. Several hand models (synthetic and digital) have been developed in the past few decades. These models ranged from physically complete and functional musculoskeletal models to simplified digital 3D scans of hand bones 
and the outer shape of the hand. For instance, the hand model from the SynDaver surgical model, which is a sophisticated full-body simulator, is a synthetic hand that simulates the mechanical and physico-chemical properties of live tissue (SynDaver Lab, Tampa, FL). Such a sophisticated synthetic model is very expensive and is not suitable for the purpose of this study which involves high levels of impact forces. A low-cost and reproducible hand model was desirable for the current study as multiple specimens were required and the specimens could not be reused due to the damage incurred by the impacts.

Another example of commercially available hand digital models is the Zygote's 3D digital model (Zygote, American Fork, UT). This model is for an adult male of $50^{\text {th }}$ percentile height and weight, and features a highly detailed hand skin and hand bones. Although this digital model may have served the needs of the current study, it was not cost effective.

Thus, a custom designed hand model was developed in this study. A set of real left hand bones (Figure 14(a)), and a high resolution laser scanner (NextEngine, Santa Monica, CA) (Figure 14(b)), both facilitated by the WVU School of Medicine, Department of Pathology, Anatomy, and Laboratory Medicine, were used to develop the hand model. Laser scans of hand bones were used to create digital images. To develop compatible soft tissues, several digital hand models were obtained from online suppliers (turbosquid.com and cgtrader.com) and were evaluated in terms of their mesh density, anatomical accuracy, multiple hand orientation options, and scalability and finally a hand model by Ubersculpts (CGTrader 3D Modeling, New York, NY) was selected. Details of the post-processing activities of the bone structure and the hand model are presented in the following sections.

In order to construct a surrogate hand that resemble the biomechanical properties of human hand, several factors were considered including: (1) the three-dimensional geometry and 
proportions of the bones and soft tissues, (2) the mechanical proprieties of the material used to represent soft and hard tissues, and (3) the relative locations of bones and soft tissues within the surrogate hand. The following sections describe these factors in detail. The design methodology is presented in three main sections including: digital phase, material selection phase, and manufacturing phase.

\subsubsection{Digital phase}

This section includes explanations of all digital activities performed toward designing the surrogate hand. The block diagram shown in Figure 12 provides a summary of the main activities performed during the digital phase.

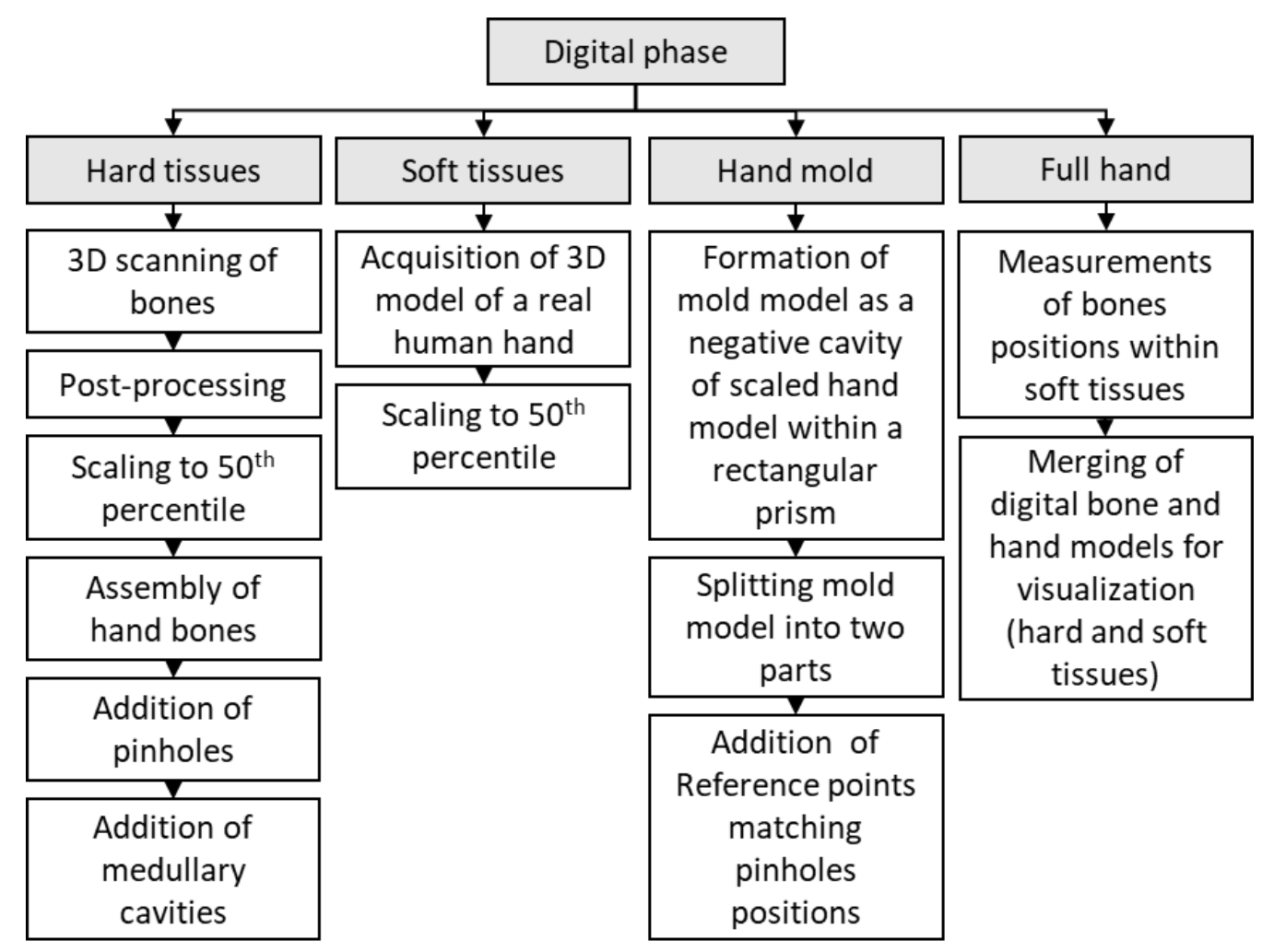

Figure 12: A summary of the main activities performed during the digital phase.

There are 27 bones in the human hand, 14 phalanges, 5 metacarpals, and 8 carpals. Each finger has 3 phalanges (distal, middle, and proximal) except the thumb, which has only 2 
phalanges. The metacarpals are the bones that make up the structure of the middle part of the hand, and the carpals are the bones that construct the wrist (Figure 13). Details of each bone were captured in the digital images of hand bones acquired using a laser scanner (Figure 14).

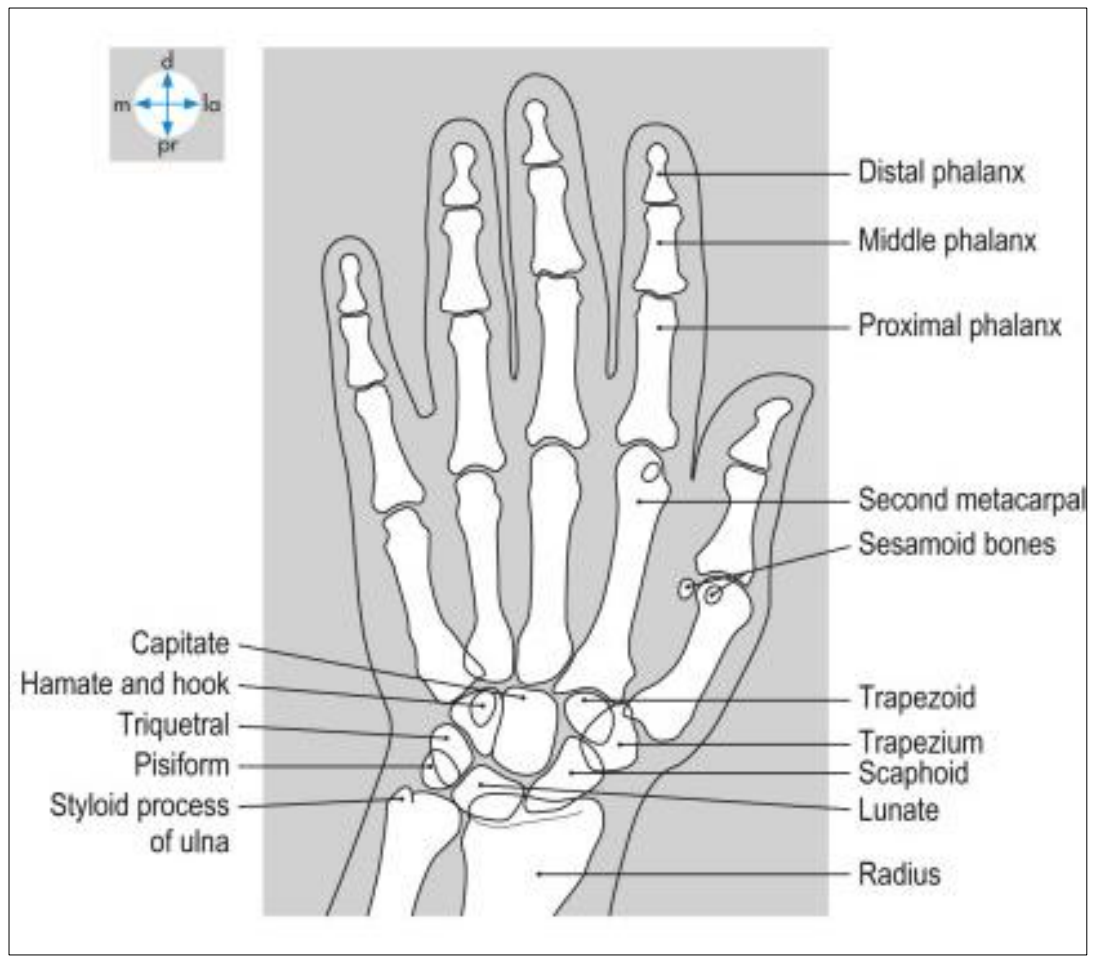

Figure 13: Hand bone anatomy (Gosling et al., 2016)

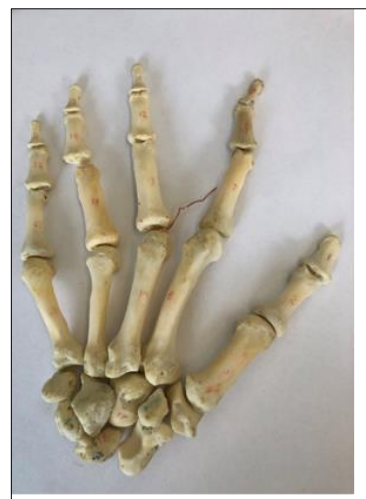

(a) Actual hand bones (left hand)

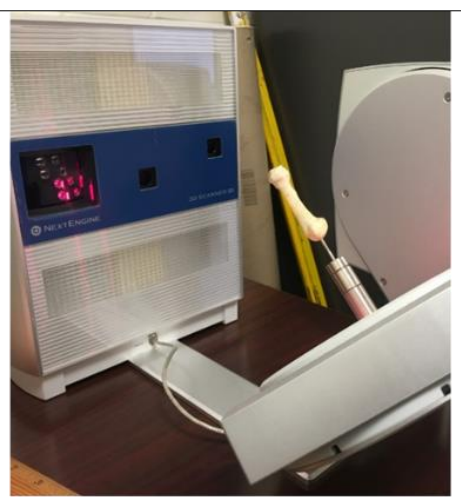

(b) 3D laser scanner

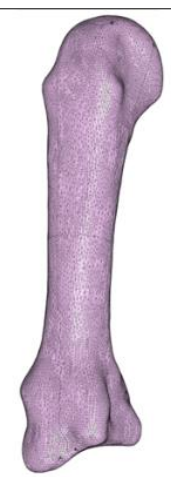

(c) Digital image of $3^{\text {rd }}$ metacarpal bone

Figure 14: Laser scanning process of the bone structure. 
The laser scanner captured multiple still images of the bone fixed on a rotary platform. These images were then assembled according to reference points marked on the bones to construct the three-dimensional external shape which was then exported as a mesh in a stereo lithography (.stl) file format (Figure 14(c)). The post-processing of bones meshes (i.e. closing the mesh body, smoothing the surfaces, and eliminating gaps and inconsistencies) was performed using the Fusion360 CAD/CAM software (Fusion360, Autodesk, San Rafael, CA).

The next step was scaling each bone to construct a bone structure that fit properly within a $50^{\text {th }}$ percentile male hand model. Previous anthropometric studies showed large variation in human hand dimensions. However, the $50^{\text {th }}$ percentile dimensions were mainly adopted for simplification purposes. Also, the choice of $50^{\text {th }}$ percentile dimensions is a common practice for surrogates (manikins) of human body parts, such as the dummies used during car testing (Louden, 2019) and the headforms used to test helmets (Liu et al., 2019). The reference $50^{\text {th }}$ percentile dimensions were obtained from X-ray images of the cadaveric hands tested during the Aim \#1 study. Out of the 13 tested cadaver hands, 5 hands had length (from wrist crease to $3^{\text {rd }}$ digit tip) and breadth (at knuckles level) similar to $50^{\text {th }}$ percentile measurements reported in the literature (Garrett, 1971; Greiner, 1991; Harrison \& Robinette, 2002) (Table 9 andTable 10). All bones measurements were performed on the posteroanterior X-ray images obtained from the specimens before the impact tests.

Table 9: Length and breadth of $50^{\text {th }}$ percentile male hand (obtained from literature) and measured from Aim \#1 study for 5 selected cadaver specimens.

\begin{tabular}{ccc}
\hline Reference & Length $[\mathrm{cm}]$ & Breadth $[\mathrm{cm}]$ \\
\hline Garrett, 1971 & 19.7 & 8.9 \\
Greiner, 1991 & 19.4 & 9.5 \\
Harrison \& Robinette, 2002 & 20.1 & - \\
\hline Average & 19.7 & 9.2 \\
SD & 0.35 & 0.42 \\
\hline
\end{tabular}


Table 10: Length and breadth of 5 selected cadaveric specimens from Aim \#1 study.

\begin{tabular}{ccc}
\hline Cadaveric hand specimen & Length [cm] & Breadth [cm] \\
\hline 1 & 19.8 & 8.7 \\
2 & 19.7 & 8.7 \\
3 & 19.2 & 9.2 \\
4 & 19.8 & 9.3 \\
5 & 19.7 & 9.2 \\
\hline Average & 19.64 & 9.02 \\
SD & 0.3 & 0.3 \\
\hline
\end{tabular}

Next, the models of the 27 bones were digitally assembled. Existing skeletal hand models (White \& Folkens, 2005) were used to assemble an anatomically accurate hand in a relaxed, nearly flat palm posture. The bones were assembled to form a flat posture similar to the posture used in the cadaveric hand study. During this stage, breakaway bone joints to connect the bones and pinholes to secure the bone structure in a mold were created. Since the wrist movement is not relevant for the purposes of this study (described in Section 3.2.2), the carpal bones were fused in the contact region except for the Trapezium to allow articulation of thumb. A small degree of flexibility/articulation of the thumb was required for putting the glove on the hand.

The next step involved digital addition of internal cavities to the metacarpals and proximal phalanges which resemble actual bone medullary cavity. The dimensions of these cavities were determined based on observations made by Fox et al. (1995). In their work, they reported the proportions of the cortical bone part and the medullary cavity from the total width of the second metacarpal bone. The average reported medullary cavity proportion of the second metacarpal of the right and left hands was generalized across all bones. Thus, the width of the medullary cavity of each bone was set to $33 \%$ of its total width. Finally, a simplified structure of radius and ulna distal end was created and connected to the bone structure (Figure 15). The assembled model 
was then mirrored by converting the image orientation to produce another model for the right hand.

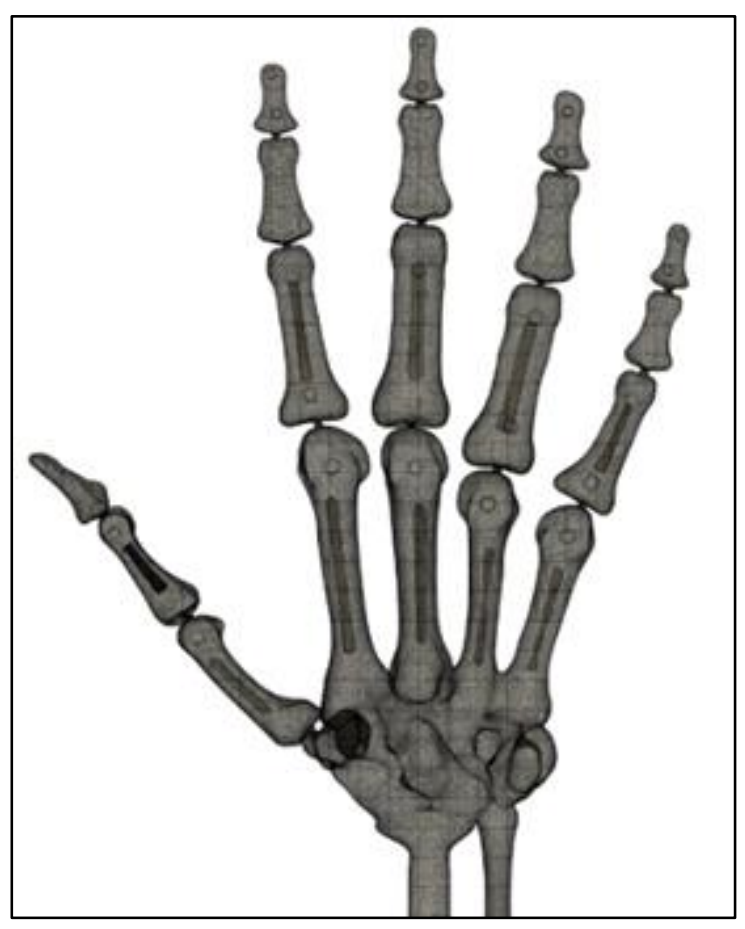

Figure 15: The digitally scaled and assembled bone structure.

The soft tissues surrounding the bones (muscles, tendons, ligaments, fat, and skin) of surrogate hand were represented as a whole, without specific distinction, and casted using medical-grade synthetic gel. The three-dimensional shape of the hand which represents the soft tissues is based on a 3D scan of a real human hand which is commercially available as a digital model (CGTrader 3D Modeling, New York, NY). This model provided accurate anatomical features, relatively flat position, high mesh density, and provisions for subsequent editing and scaling. Some minor digital modifications were made to this model to make it comparable with $50^{\text {th }}$ percentile male hand. 
Garrett (1971) reported that the hand breadth and length of $50^{\text {th }}$ percentile male air force flight personnel were $8.96 \mathrm{~cm}$ and $19.72 \mathrm{~cm}$, respectively. The same dimensions of $50^{\text {th }}$ percentile male U.S. army personnel were $9.53 \mathrm{~cm}$ and $19.41 \mathrm{~cm}$, respectively (Greiner, 1991). Also, Harrison \& Robinette (2002) reported that the length of $50^{\text {th }}$ percentile male of general U.S. population were $20.10 \mathrm{~cm}$ (Table 9). The scaled digital hand model used in the current study has breadth and length of $9.24 \mathrm{~cm}$ and $19.52 \mathrm{~cm}$, respectively (Figure 16), which are nearly identical to the average of the data reported in the literature (Garrett, 1971; Greiner, 1991; Harrison \& Robinette, 2002) (Table 9).

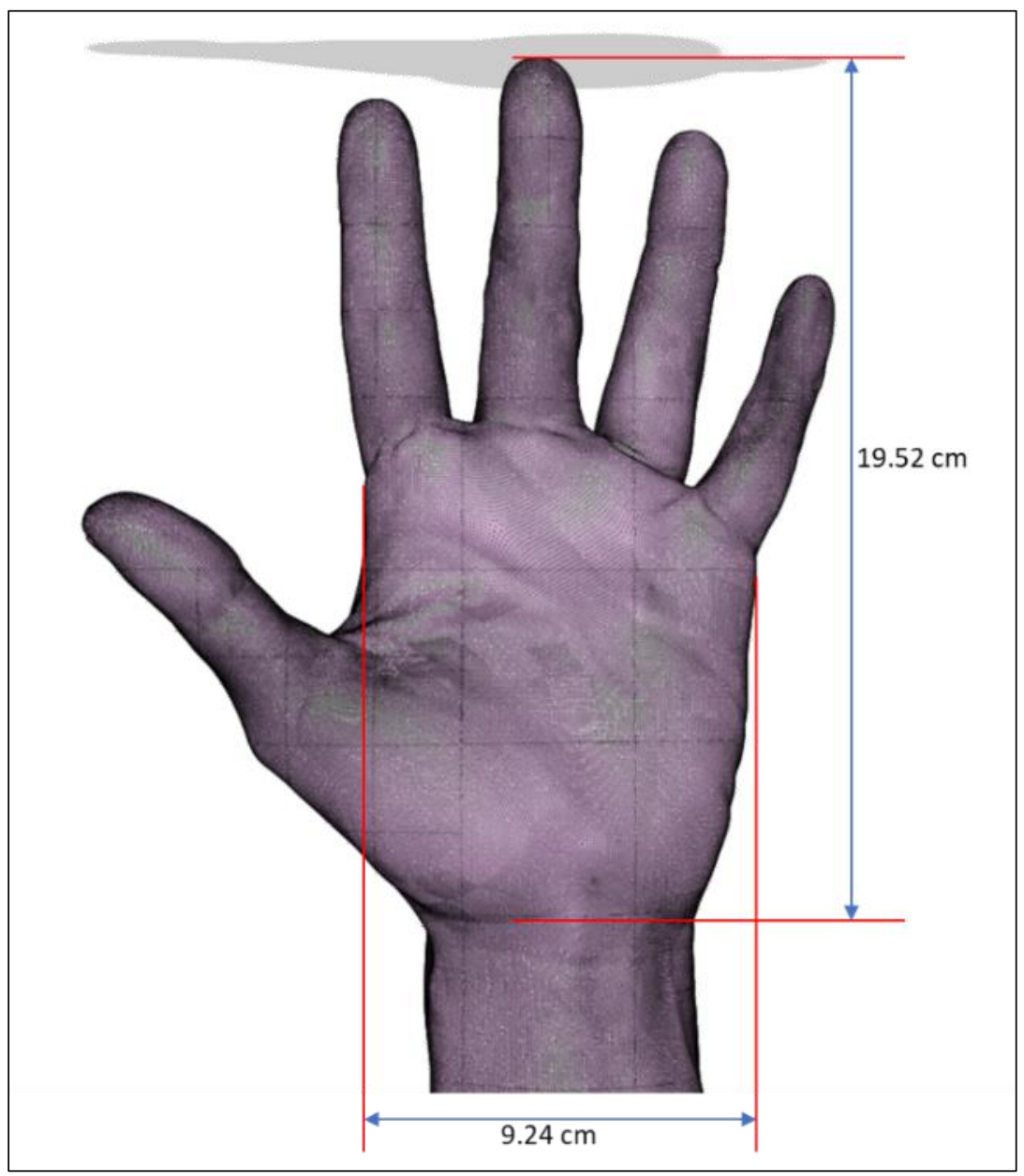

Figure 16: The scaled hand digital model. 
The next step consisted of digitally combining the assembled and scaled bone structure, and the three-dimensional shape of the hand (Figure 17). Soft tissues in the human hand (as a whole, without distinction of specific tissues) are not distributed evenly above and below the bones. The placement of the bone structure within the soft tissues in the surrogate hand was approximated according to measurements performed on X-ray images (oblique view) of cadaveric hands (Aim \#1) and published MR images (Clavero et al., 2003). The thicknesses of the soft tissues above and below the bone structure at the levels of the previously created pinholes were measured. These measurements were used to instrument the mold with supporting wooden pins, which represent soft tissue thickness, at the reference points to maintain the same placement of bone structure within the soft tissues.

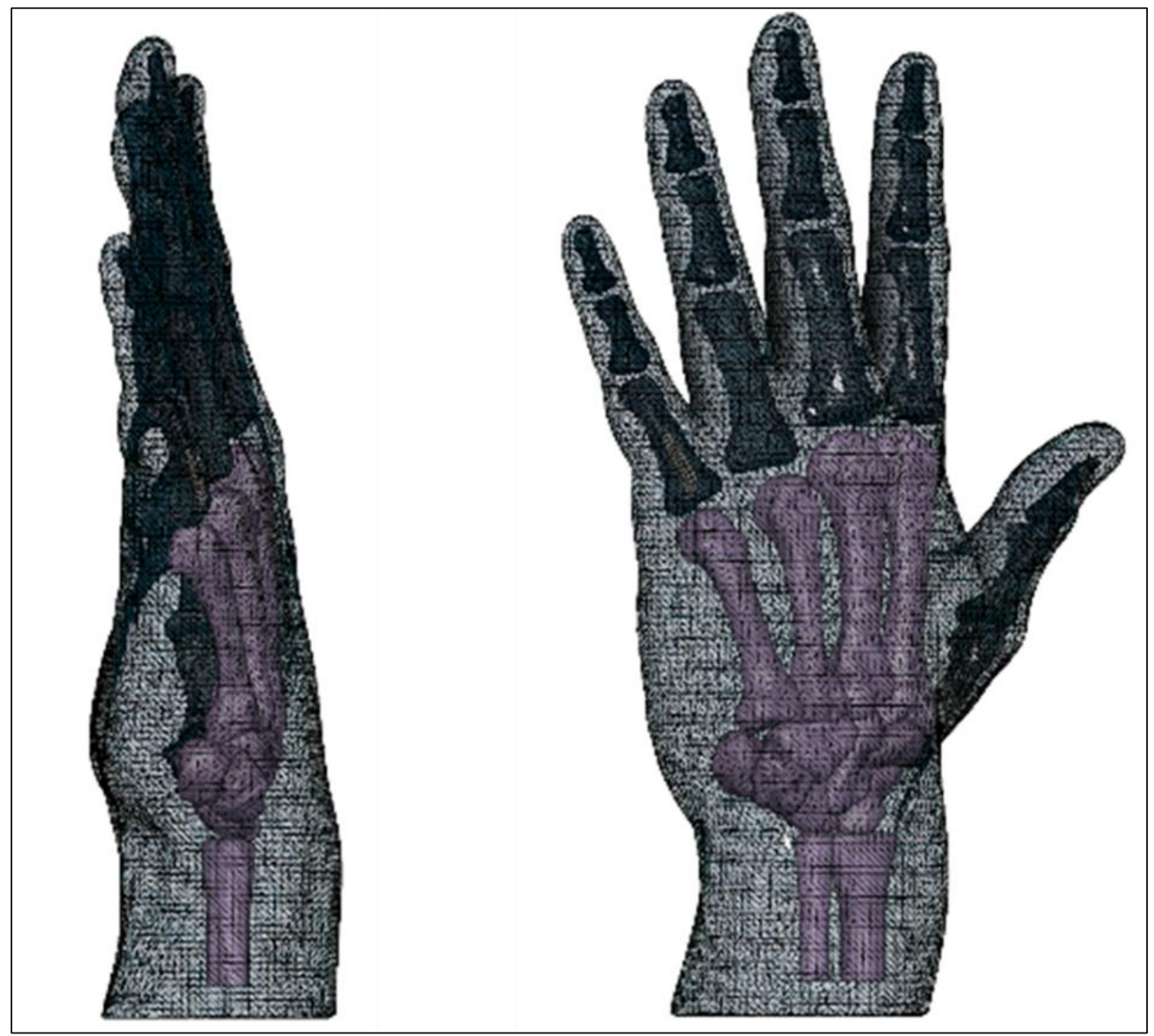

Figure 17: Digital hand shape and bone structure. 
The hand model developed in the previous step was used as a negative cavity within a rectangular prism to produce a digital model for a mold (Figure 18). This prism was split into two parts by a reference plane dividing the mold body into two separate parts. The position of the reference plane was carefully selected to allow for easy cast removal from the mold without distorting the shape of the hand. The mold was instrumented with reference points at the palmar and dorsal sides matching the positions of the pinholes created into the bone structure. The pinholes (on bone structure) and reference points (on mold) were used during the manufacturing phase to ensure accurate and consistent positioning of the bone structure within the mold. Also, leader pins and slots were created in the design of the mold to provide accurate alignment between the mold parts. The mold was designed with fingers pointing downward and the wrist open to facilitate the gel casting process and to minimize the formation of air pockets within the cavity (Figure 18).

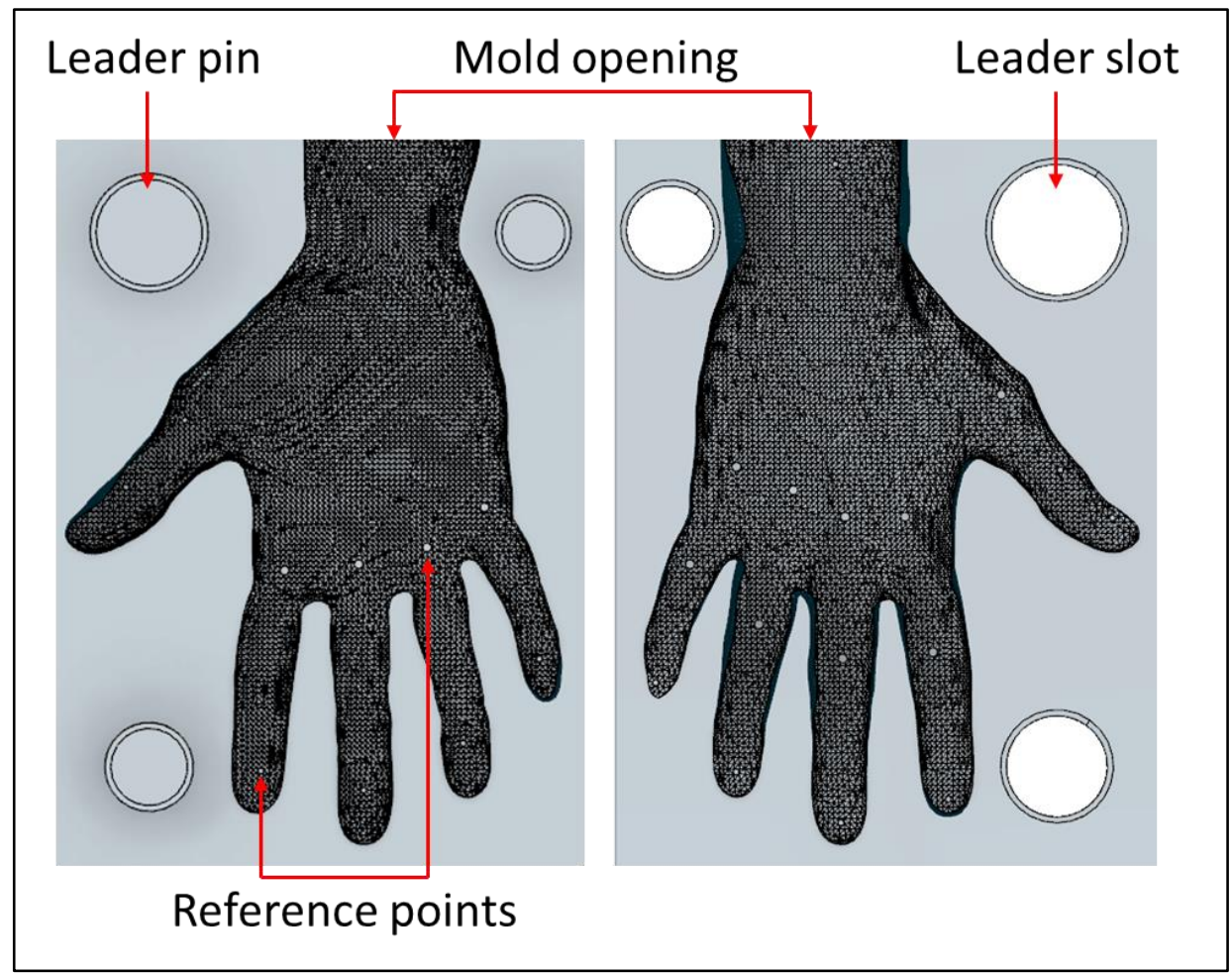

Figure 18: Hand mold model. 


\subsubsection{Material selection}

This section presents the steps and considerations followed to select materials used in surrogate hand construction. The block diagram shown in Figure 19 provides a summary of the main activities performed within this section.

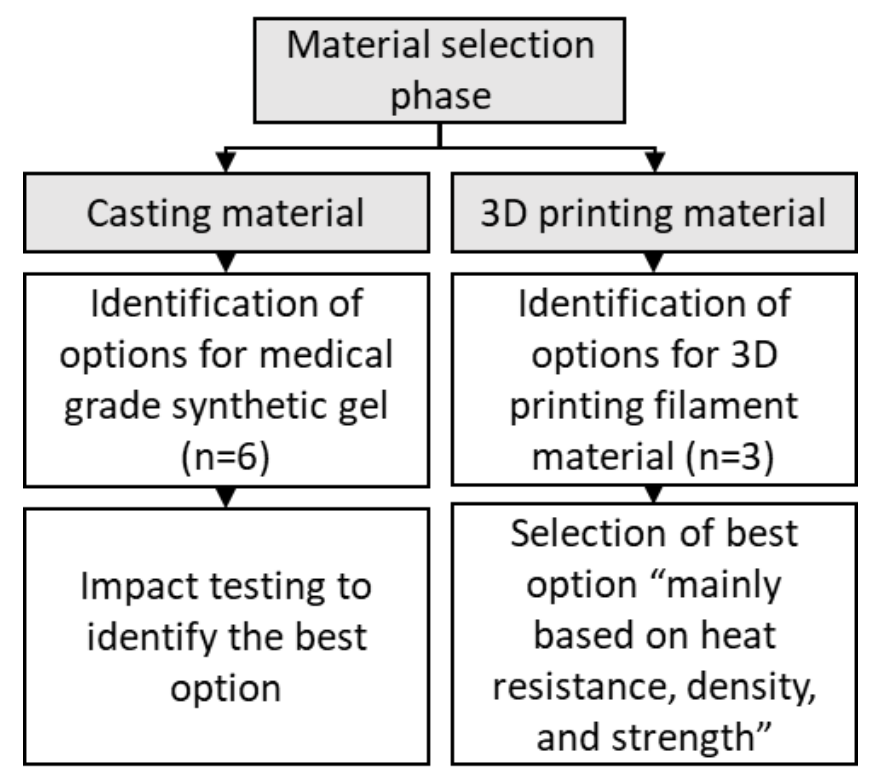

Figure 19: A summary of the main activities performed within the material selection section.

The soft tissues surrounding the 3D printed bone structure was casted using medical-grade synthetic gel (Humimic Medical, Fort Smith, AR). This gel is commercially available in six levels of hardness and advertised to have haptic response similar to different types of human tissues (Table 11). Previous studies have used this synthetic gel to construct high-fidelity tactile surrogates to human body parts for medical training (Headman et al., 2020; Pang et al., 2020; Risler et al., 2018; Y. Y. Wu et al., 2016, 2018).

As per the manufacturer's specifications, the solid state melting temperature, density and hardness measured by the Shore 00 scale for the gels are in the range of $116^{\circ} \mathrm{C}$ to $121^{\circ} \mathrm{C}, 834.34$ 
to $981.63 \mathrm{Kg} / \mathrm{m}^{3}$, and 3.3 to 21.4 , respectively (Table 11). The Shore hardness can be measured using a Durometer, which measures the resistance of plastics to indentation in a scale from 0 to 100. Higher numbers on the scale indicate a higher resistance to indentation and thus, a harder material, while lower numbers mean less indentation resistance and typically correspond to softer materials (Mix \& Giacomin, 2011).

After initial evaluation of stiffness and hardness, Gelatin \#5 was excluded as its structure was extremely soft. Also, Gelatin \#1 was excluded as its Shore rating and manufacturer's notes were very close to Gelatin \#0. Gelatins \#0, \#2, \#3 and \#4 were evaluated under impact loads and compared to data obtained from Aim \#1 study. This is explained later in Section 4.2.2.

Table 11: Synthetic gel proprieties. Shore rating is on the Shore 00 standard (Humimic Medical, Fort Smith, AR).

\begin{tabular}{cccc}
\hline Gel grade & Density $\left[\mathrm{Kg} / \mathrm{m}^{3}\right]$ & Shore rating (avg.) & Manufacturer's note \\
\hline Gelatin \#0 & 880.38 & 21.4 & $\begin{array}{c}\text { Simulates thigh } \\
\text { muscles, biceps, and } \\
\text { back muscles }\end{array}$ \\
\hline Gelatin \#1 & 936.48 & 17.8 & $\begin{array}{c}\text { Simulates neck } \\
\text { muscles, healthy skin, } \\
\text { liver, and heart }\end{array}$ \\
\hline Gelatin \#2 & 923.47 & 6.8 & $\begin{array}{c}\text { Simulates skin, } \\
\text { muscles, and lung } \\
\text { tissue }\end{array}$ \\
Gelatin \#3 & 981.63 & 4.6 & $\begin{array}{c}\text { Simulates fatty tissue } \\
\text { Gelatin \#4 }\end{array}$ \\
\hline Gelatin \#5 & 834.34 & 3.3 & $\begin{array}{c}\text { Simulates the feel of a } \\
\text { breast tissue, intestinal } \\
\text { tissue, and } \\
\text { subcutaneous fat }\end{array}$ \\
\hline & 898.45 & Not reported & $\begin{array}{c}\text { Simulates blood clots } \\
\text { and brain tissue }\end{array}$ \\
\hline
\end{tabular}


On the other side, 3D printing materials, in the filament form for Filament Fusion Fabrication (FFF) printers, are generally comprised of thermo-plastics, metals, composites, ceramics, or biomaterials, or a combination of multiple components such as a mixture of bio-ceramics and polymers. Each material type or mixture has its own unique proprieties such as the strength, density, and heat resistance. The factors that were given priority for selecting the 3D printing material were: (1) the ability to withstand the melting temperature $\left(121^{\circ} \mathrm{C}\right)$ of the synthetic gel used for soft tissues (2) strength and density comparable with human bones, (3) availability, (4) price, and (5) printability with a non-specialized 3D printer.

The initial materials considered were Acrylonitrile Butadiene Styrene (ABS; Makeshaper, Barberton, OH), Polylactic Acid (PLA; Makeshaper, Barberton, OH), and Nylon 6 (PA6; Nylstrong by Smartfil, Spain). Although all the selected materials had melting temperatures higher than $121^{\circ} \mathrm{C}$, it was anticipated that some distortion could occur when dealing with small 3D printed parts (particularly with the smaller phalangeal bones). Therefore, a simplified heat resistance test was conducted using 3D printed samples of the proximal phalanx of $3^{\text {rd }}$ finger (Figure 20). The 3D printed bones using the 3 selected materials (5 samples of each material) were embedded in a container filled with molten synthetic gel $\left(121^{\circ} \mathrm{C}\right)$. After allowing 24 hours for cooling and full solidification of the gel, the specimens of each sample were removed from the gel and the length of each specimen was measured and compared with the pre-test length (Figure 20). Negligible dimension changes in the samples created using ABS and Nylon 6 were observed (Table 12). However, a change in the length was observed for the sample created using PLA (i.e. 3.55\% shrinkage) (Table 12). This change in length was attributed to (1) PLA melting temperature (i.e. range from $130^{\circ} \mathrm{C}$ to $180^{\circ} \mathrm{C}$ ) being very close to gel melting temperature (i.e. $121^{\circ} \mathrm{C}$ ), and (2) the size of the tested 3D printed parts was very small. 


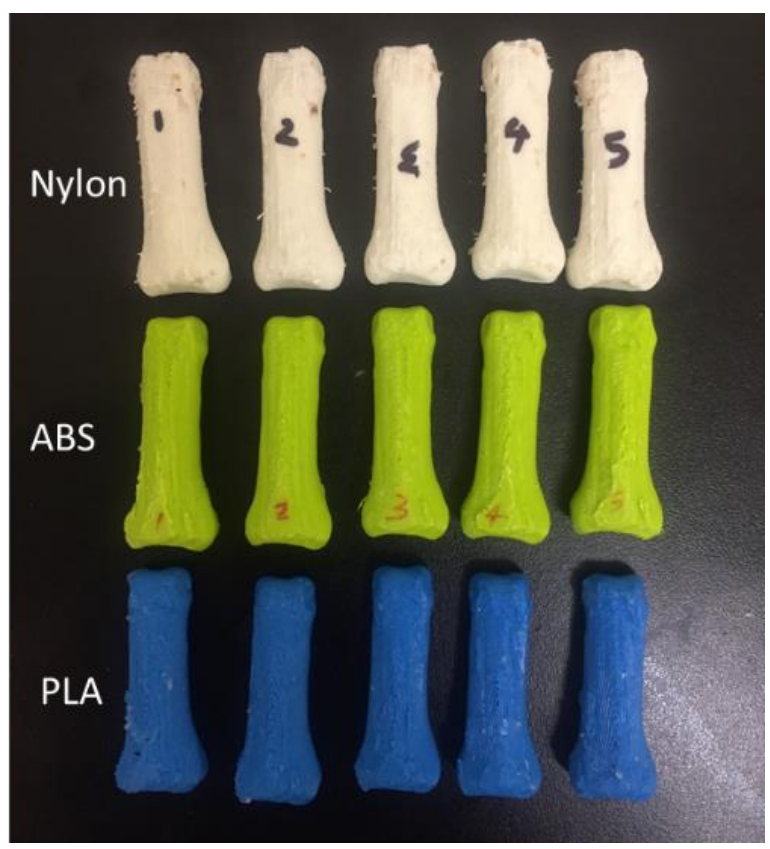

Figure 20: Heat resistance test using molten synthetic gel. Bones on the left side are without heat resistance test. Bones on the right side are after heat resistance test.

Table 12: Results of the heat resistance test using molten synthetic gel on 3D printed bones using different materials.

\begin{tabular}{c|ccc|ccc|ccc}
\hline Material & \multicolumn{3}{|c|}{ Nylon } & \multicolumn{3}{c|}{ ABS } & \multicolumn{3}{c}{ PLA } \\
\hline \multirow{3}{*}{ Sample } & $\begin{array}{c}\text { Original } \\
\text { length } \\
{[\mathrm{mm}]}\end{array}$ & $\begin{array}{c}\text { After } \\
\text { test } \\
{[\mathrm{mm}]}\end{array}$ & $\begin{array}{c}\text { Change } \\
\%\end{array}$ & $\begin{array}{c}\text { Original } \\
\text { length } \\
{[\mathrm{mm}]}\end{array}$ & $\begin{array}{c}\text { After } \\
\text { test } \\
{[\mathrm{mm}]}\end{array}$ & $\begin{array}{c}\text { Change } \\
\%\end{array}$ & $\begin{array}{c}\text { Original } \\
\text { length } \\
{[\mathrm{mm}]}\end{array}$ & $\begin{array}{c}\text { After } \\
\text { test } \\
{[\mathrm{mm}]}\end{array}$ & $\begin{array}{c}\text { Change } \\
\%\end{array}$ \\
\hline 1 & 41.65 & 41.46 & 0.46 & 41.19 & 41.34 & -0.36 & 41.33 & 39.99 & 3.24 \\
\hline 2 & 41.60 & 41.65 & -0.12 & 41.12 & 41.08 & 0.10 & 41.45 & 40.08 & 3.31 \\
\hline 3 & 41.65 & 41.41 & 0.58 & 41.10 & 41.12 & -0.05 & 41.38 & 39.95 & 3.46 \\
\hline 4 & 41.64 & 41.48 & 0.38 & 41.08 & 40.92 & 0.39 & 41.46 & 39.82 & 3.96 \\
\hline Avg. & 41.59 & 41.51 & 0.19 & 41.10 & 41.09 & 0.02 & 41.49 & 39.91 & 3.81 \\
\hline & 41.63 & 41.50 & 0.30 & 41.12 & 41.11 & 0.02 & 41.42 & 39.95 & 3.55 \\
\hline
\end{tabular}

The second important factor for selecting the 3D printing material was having strength and density comparable with human bones. Most of the hand bones are long bones with a shaft and two ends. The shell of the shaft is made of cortical bone tissues (Figure 21). Human cortical bone density and bending strength are reported to be $1.9 \mathrm{~g} / \mathrm{cm}^{3}$ (Öchsner et al., 2011) and $164 \mathrm{MPa}$ (SD 29) (Reilly \& Burstein, 1974), respectively. Out of the remaining two filament materials 
(ABS and Nylon 6), the Nylon filament (PA6; Nylstrong by Smartfil, Spain), was selected as its mechanical properties (density $=1.52 \mathrm{~g} / \mathrm{cm}^{3}$, bending strength $=120 \mathrm{MPa}$, and thermal resistance $=210^{\circ} \mathrm{C}$ ) were the closest to bone properties (a copy of the filament datasheet is included in Appendix C).

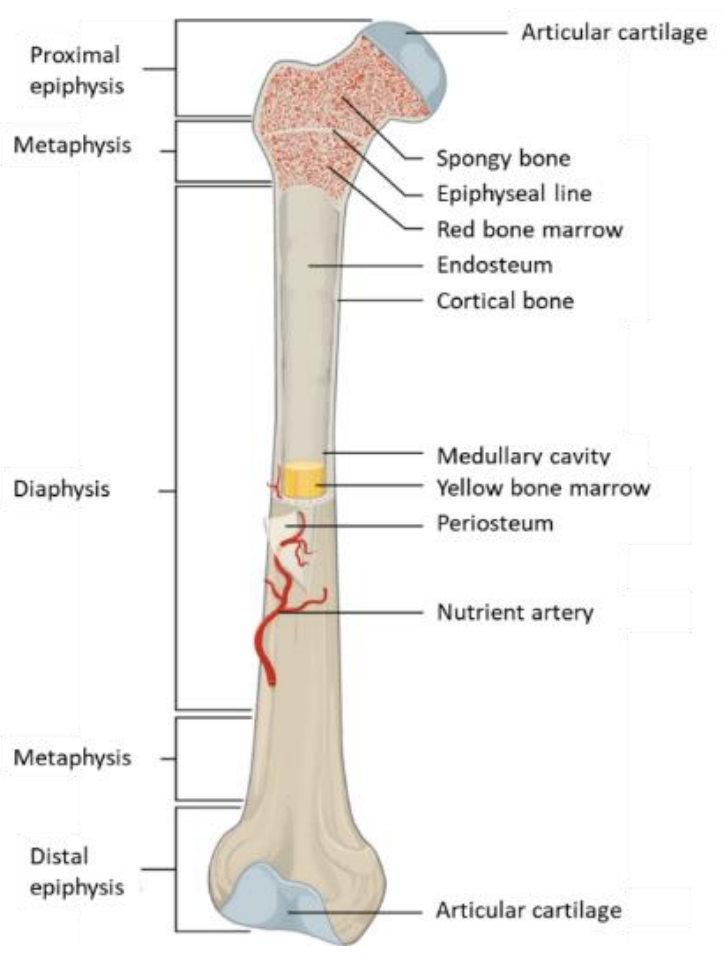

Figure 21: Anatomy of a long bone (OpenStax, 2017)

\subsubsection{Manufacturing phase}

This section details the steps followed during manufacturing phase. The block diagram shown in Figure 22 provides a summary of the sub-sections within the manufacturing phase section and the main activities performed. 


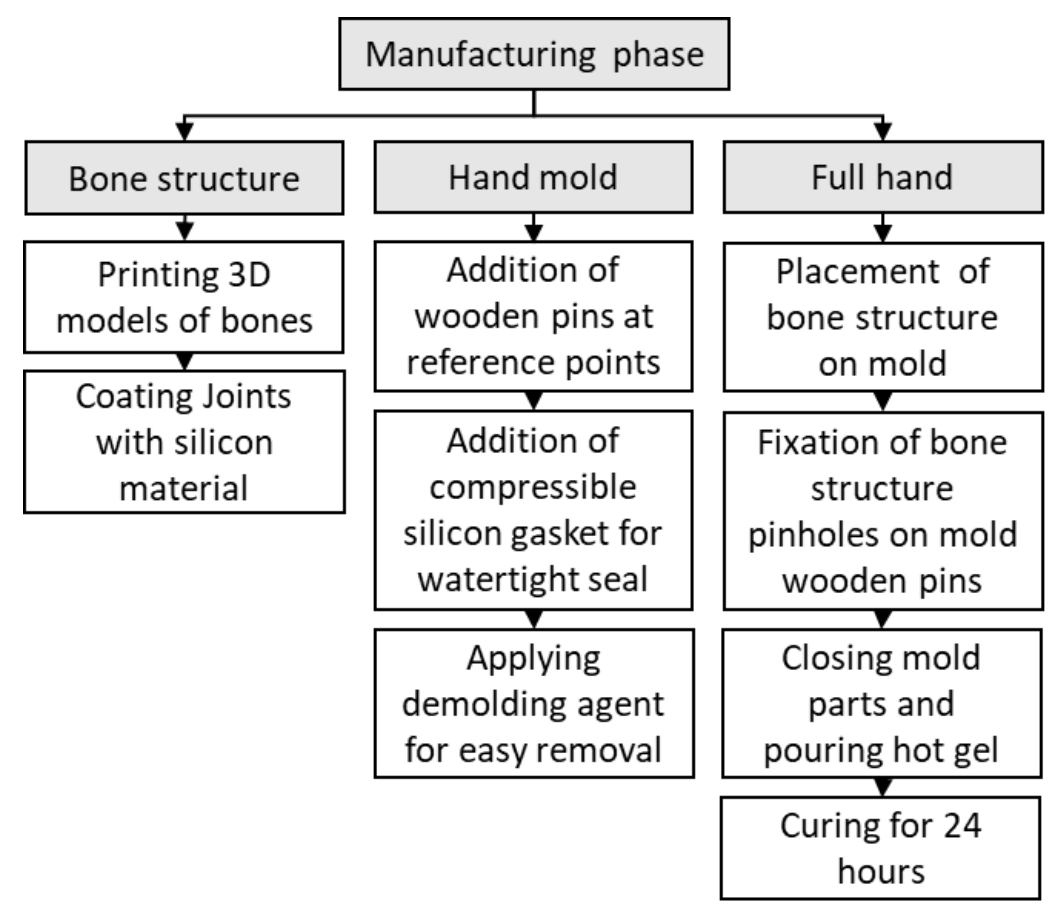

Figure 22: A summary of the sub-sections within the manufacturing Phase section and the main activities performed.

\subsection{Bone Structure}

The final design of the scaled digital bone structure was uploaded into a slicer program that converted it to a printable file (.gcode format) recognizable by a FFF 3D printing machine (LulzBot TAZ Pro, Aleph Objects, Loveland, CO). This machine manufactures a desired part by laying down layers of molten material extruded through a heated nozzle (Figure 23). The slicer program allows the user to control several printing parameters which, in addition to the material properties, could significantly influence the strength of the printed part. Specifically, the number of perimeters, the number of top and bottom layers, and the infill pattern and density (Figure 24 and Figure 25) were reported to significantly affect the strength of the printed object (FernandezVicente et al., 2016; Lanzotti et al., 2015). For a given geometry, the number of perimeters is the number of shells that construct the exterior of the printed part. The infill pattern and density are the geometrical shape and the amount of material printed inside the printed part. 


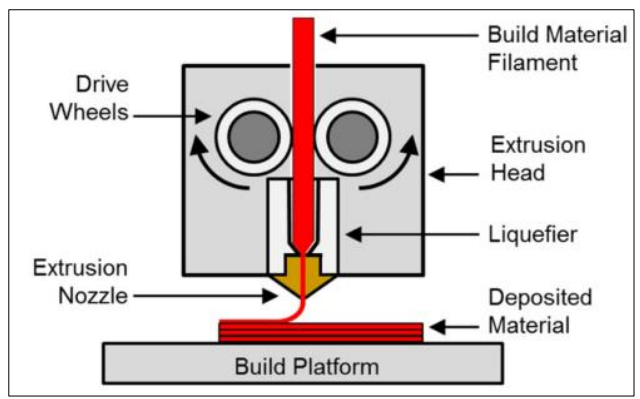

Figure 23: Schematic of standard FDM machining process (Cantrell et al., 2017).

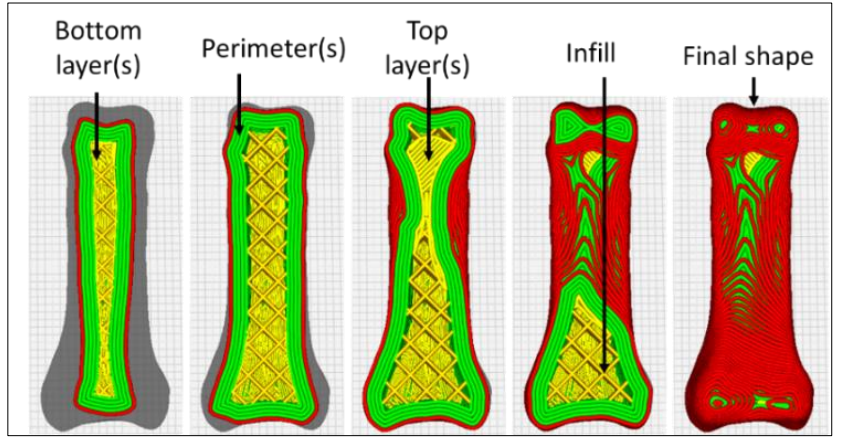

Figure 24: Slicing settings and visualization of layers build up in a slicer program.

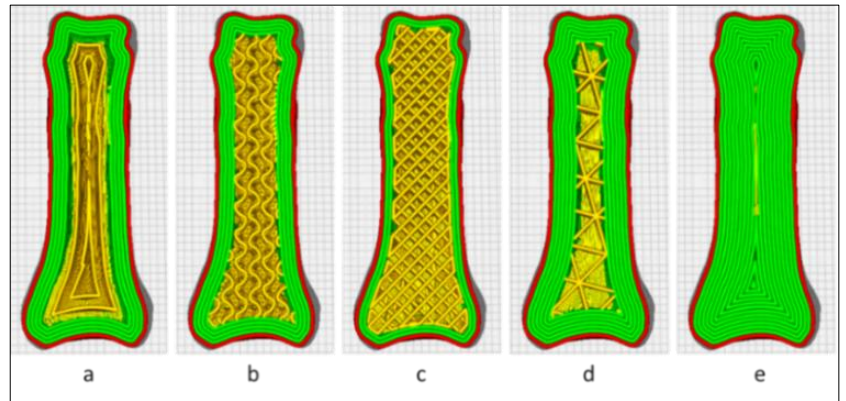

Figure 25: Cross sections of a G-code of the same bone in a slicer program with different settings of infill shape and number of perimeters. (a) Concentric infill and 5 perimeters. (b) Gyroid infill and 5 perimeters. (c) Zig Zag infill and 3 perimeters. (d) Triangular infill and 7 perimeters. (e) Solid part created by concentric perimeters and no infill.

Given the complex structure of the human hand which comprises hard and soft tissues with different mechanical properties, multiple combinations of printing parameters were considered in order to achieve comparable global stiffness. After completion of the 3D printing process, the finger joints were coated with silicon material to mimic ligaments. The selected silicon material can withstand the melting temperature $\left(121^{\circ} \mathrm{C}\right)$ of the synthetic gel used for soft tissues. The support material was then removed to obtain the fully assembled bone structure (Figure 26). 


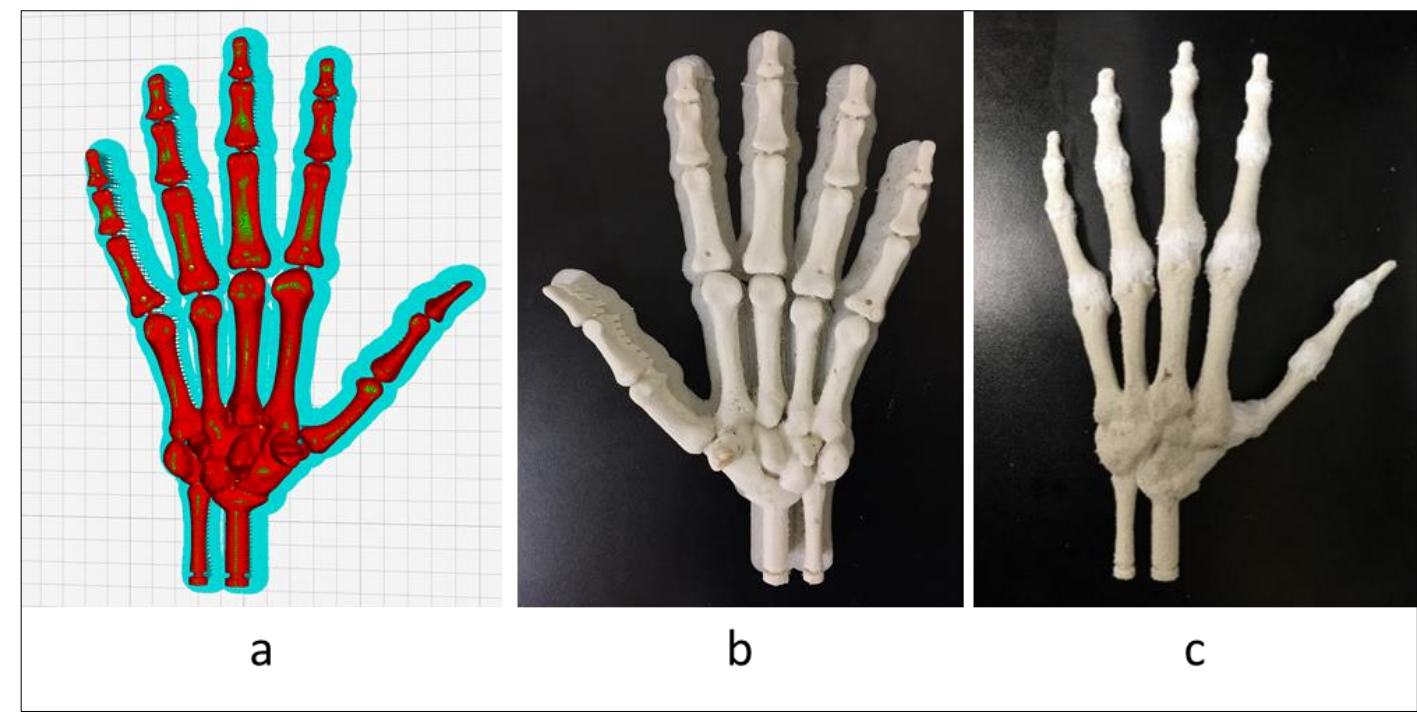

Figure 26: (a) Bone structure in slicing software; (b) 3D printed bone structure with support material (palmar view); (c) Finished 3D printed bone structure with silicon material joining bones (dorsal view).

The initial printing settings were set to generate bones with three main structural components including: (1) 5 perimeters that resemble the cortical part of long bones, (2) cavity that resemble long bones medullary cavity (added during digital phase), and (3) Zig Zag infill pattern with 50\% density which resemble spongy bones (Figure 27$)$. The resulting bone structure ( $1^{\text {st }}$ generation) was used to manufacture the first prototype of surrogate hand, which was utilized to evaluate different synthetic gel grades and to fine tune the printing settings based on the results of the impact tests.

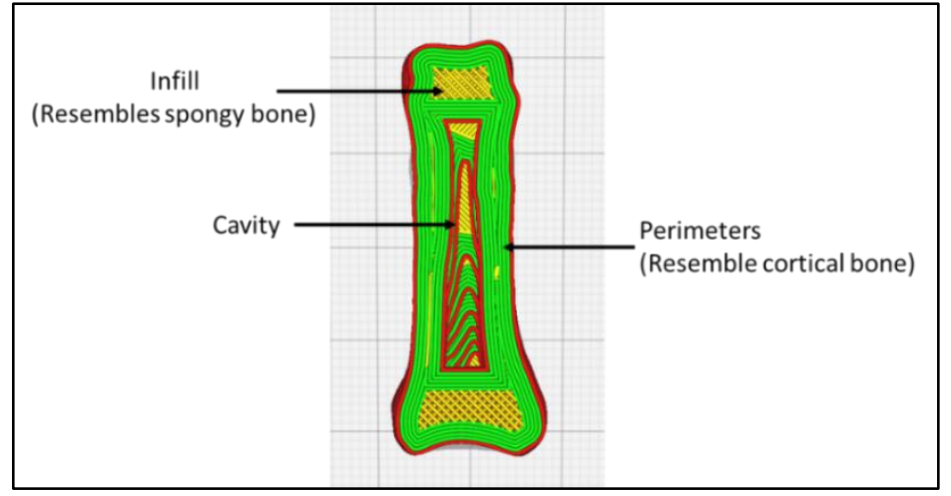

Figure 27: Initial 3D printing settings (used for $1^{\text {st }}$ generation of bone structure). 
Impact response data from surrogate hands manufactured using Gelatin \#4 were the closest to impact response data from cadaveric hands. Therefore, all subsequent tests were performed using surrogate hands manufactured using Gelatin \#4. Surrogate hands constructed using the $1^{\text {st }}$ generation of bone structure and Gelatin \#4 were referred to as "Gel \#4.1". Based on the impact response data generated by testing Gel \#4.1 surrogate hands, an iterative adjustment process was performed by changing $3 \mathrm{D}$ printing settings and then evaluating impact response data relative to cadaveric hands data. This adjustment process yielded two more generations of bone structures (i.e., $2^{\text {nd }}$ and $3^{\text {rd }}$ generations). The changes implemented in each generation were directly based on the results obtained from impact tests of surrogate hands manufactured using the prior generation. The modifications only included changing the 3D printing settings to improve global stiffness of the surrogate hand.

The impact reaction forces obtained from Gel \#4.1 were distant from cadaveric hands data. Also, a thorough evaluation of the impacted bone structures showed signs of layer separation and weak points, mainly caused by the complexity of the added cavities. Therefore, bone cavities were removed from the digital files of the bone structure. Instead, an infill geometrical pattern provided by the slicer program was used. The choice of infill design was based on a pilot study performed by impacting 3D printed single bones with different infill patterns, and then evaluating the resulted reaction force and the presence of weak points. The concentric infill pattern provided better reaction force values and a general structure similar to human long bones with simplified cavities (Figure 25(a)). Thus, the printing parameters for the $2^{\text {nd }}$ generation of bone structure were set as follow: 2 perimeters, 2 top and bottom layers, and concentric infill pattern with $20 \%$ density. Surrogate hands constructed using the $2^{\text {nd }}$ generation of bone structure and Gelatin \#4 were referred to as "Gel \#4.2". 
Results of Gel \#4.2 impact testing showed a great improvement in the impact reaction forces on the PIP position. However, minimal improvement was observed in the MCP and Metacarpals positions. Therefore, the digital file of the bone structure was divided into two separate files. One file for the fingers and the other for the wrist and metacarpal bones (Figure 28). Such separation facilitates modification of parts that require additional improvement (i.e. MCP and metacarpals) without altering the whole model. Additional geometry components were generated to facilitate the articulation of the bones with breakaway supports to maintain the bone orientation and reduce removal of support material. Also, the radius and ulna distal end structure was improved. The printing parameters for the fingers part of the $3^{\text {rd }}$ generation of bone structure (Figure 28 (b)) were kept the same as the $2^{\text {nd }}$ generation, as they provided good results. Impact reaction forces on the MCP and Metacarpals positions, from Gel \#4.2 surrogate hand, suggested that an extra strength in the bone structure was required. Therefore, the printing parameters of the metacarpals part of the $3^{\text {rd }}$ generation of bone structure (Figure 28 (a)) were modified to the following: 5 perimeters, 2 top and bottom layers, and concentric infill pattern with 25\% density. Surrogate hands constructed using the $3^{\text {rd }}$ generation of bone structure and Gelatin \#4 were referred to as “Gel \#4.3”.

Table 13 summarizes the 3D printing settings of the different generations. The development process of bone structures and surrogate hands is illustrated in Figure 29. Results of impact test performed during the improvement process are shown below in Section 4.2.6. 


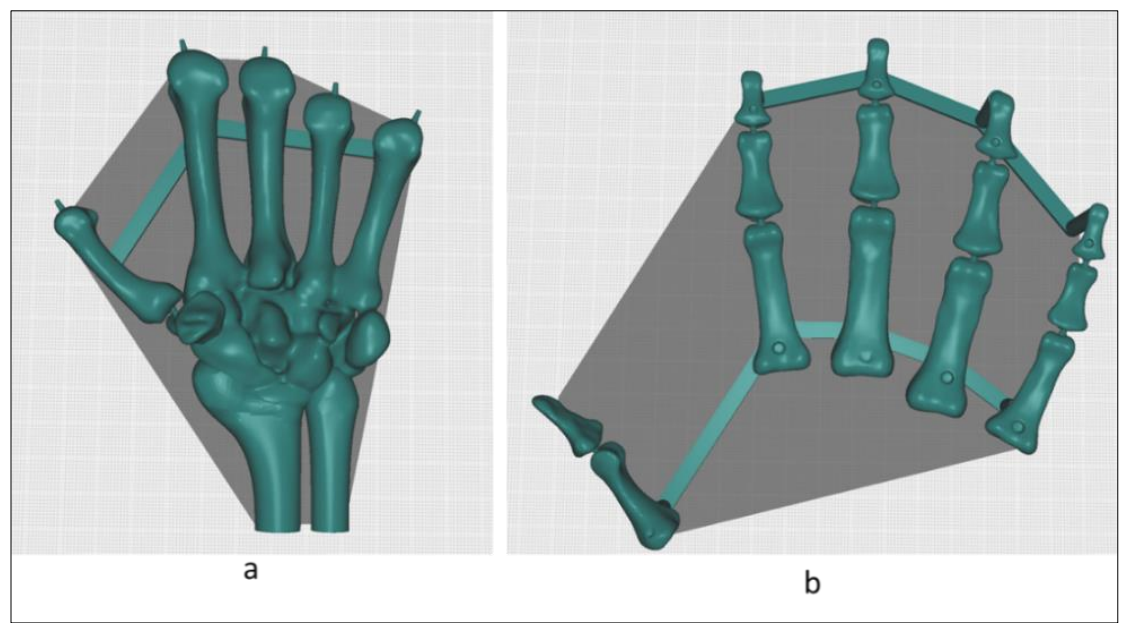

Figure 28: Bone structure in slicing software with the modification implemented for the surrogate hand generation Gel \#4.3. (a) Metacarpals and carpals portion; (b) Phalangeal portion.

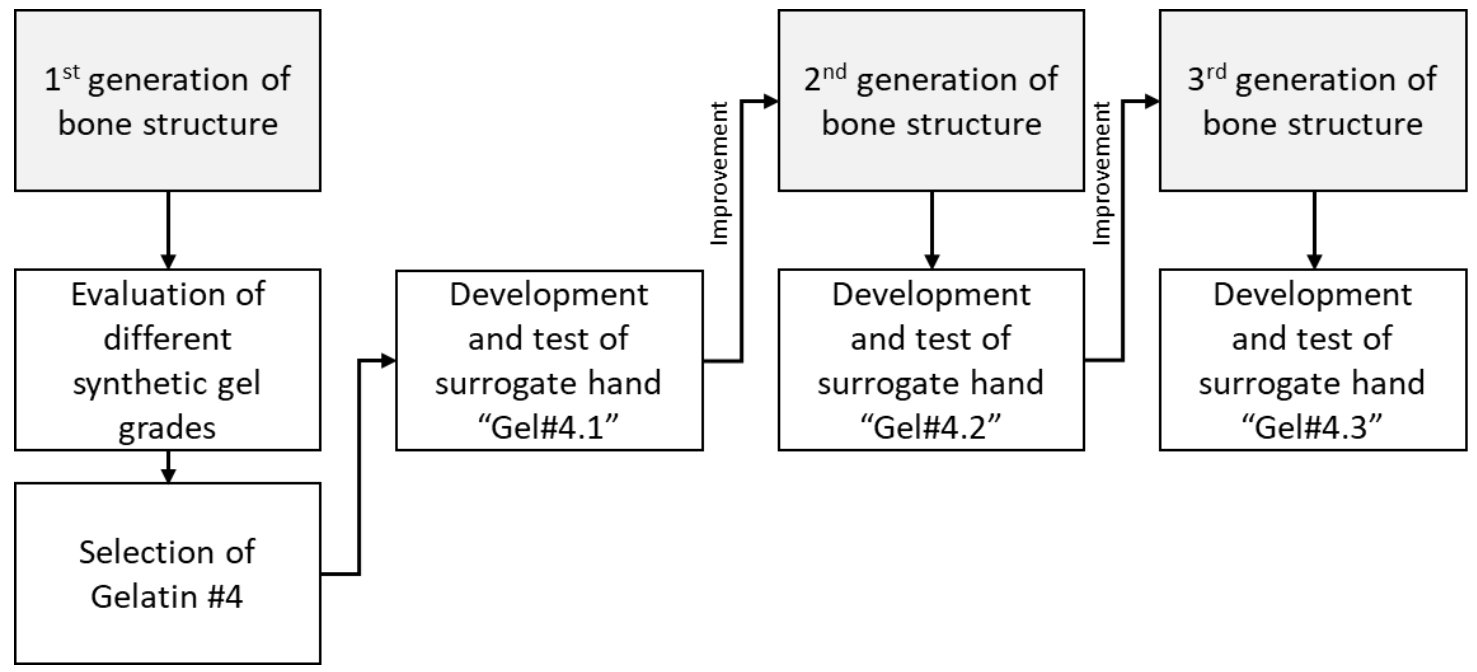

Figure 29: A summary of the process followed during the manufacturing and development of bone structures and surrogate hands.

Table 13: summary of 3D printing settings used in printing bone structures of different generations of surrogate hands.

\begin{tabular}{c|c|c|c|c}
\hline Bone structure & $\begin{array}{c}1^{\text {st }} \\
\text { generation }\end{array}$ & $\begin{array}{c}2^{\text {nd }} \\
\text { generation }\end{array}$ & $\begin{array}{c}3^{\text {rd }} \text { generation } \\
\text { (Fingers) }\end{array}$ & $\begin{array}{c}3^{\text {rd }} \text { generation } \\
\text { (Metacarpals) }\end{array}$ \\
\hline Printing temperature $\left[\mathrm{C}^{\circ}\right]$ & 265 & 265 & 265 & 265 \\
\hline Printing speed $[\mathrm{mm} / \mathrm{sec}]$ & 20 & 20 & 20 & 20 \\
\hline Nozzle Diameter $[\mathrm{mm}]$ & 0.5 & 0.5 & 0.5 & 0.5 \\
\hline Layer height [mm] & 0.2 & 0.2 & 0.2 & 0.35 \\
\hline Number of perimeters & 5 & 2 & 2 & 5 \\
\hline Infill pattern and density & Zig Zag; & Concentric; & Concentric; & Concentric; \\
\hline Number of top and bottom layers & $50 \%$ & $20 \%$ & $20 \%$ & $25 \%$ \\
\hline
\end{tabular}




\subsection{Hand mold and casting}

The two-part 3D printed mold was instrumented with wooden pins at reference points with height that represent thicknesses of soft tissue. A compressible silicon gasket was also placed between the mold parts to ensure a watertight seal during the gel casting (Figure 30 (a)). To allow for easy removal of the surrogate hand from the mold, the two-part mold was also coated with a demolding agent before casting gel.

Once the bone silicon joints were cured, the bone structure was placed in the mold, supported by the wooden pins and pinholes to secure bone position within the hand cavity (Figure 30 (a)). The two parts of the mold were then assembled and held using bar clamps (Figure 31). The synthetic gel was heated to $121^{\circ} \mathrm{C}$ and the liquid gel was poured into the mold while tilting the mold side to side to ensure smooth flow of material and removal of air out of the hand cavity. Subsequently, percussive assistance was applied to complete the degassing of the gel as it cooled down. The resulting cast was left to cool down and solidify for 24 hours before demolding. The cast hand after demolding one part of the mold, and the finished surrogate hand are shown in Figure 30 (b) and (c). The final design of bone structure $\left(3^{\text {rd }}\right.$ generation $)$ and casted right and left Gel \#4.3 surrogate hands are shown in Figure 32. 


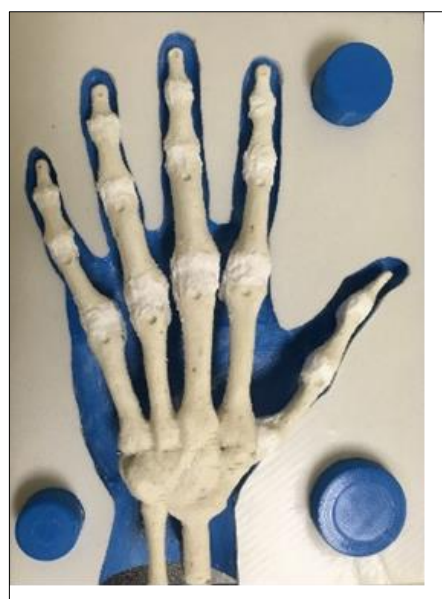

a

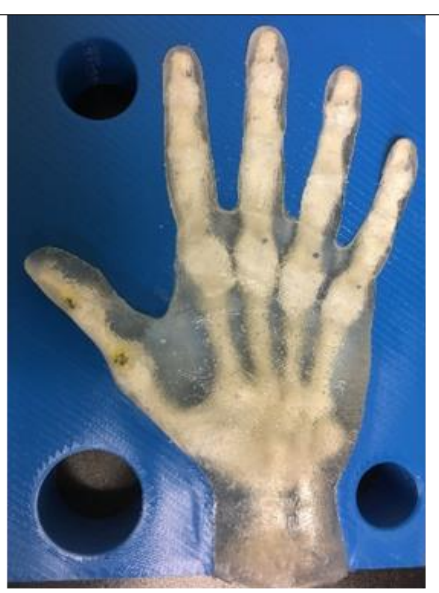

b

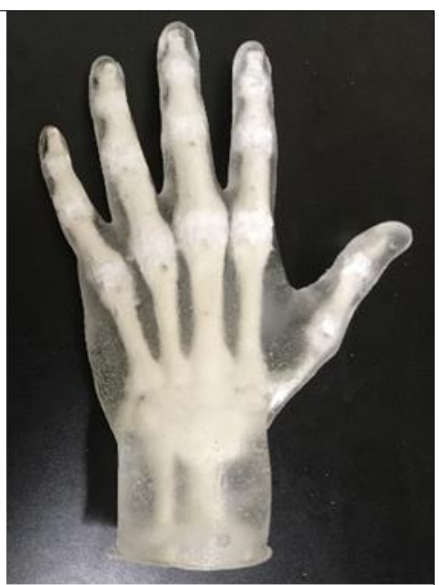

C

Figure 30: (a) 3D printed bone assembly placement on mold; (b) Palmar view of gel hand after removing one half; (c) Dorsal view of finished surrogate gel hand.

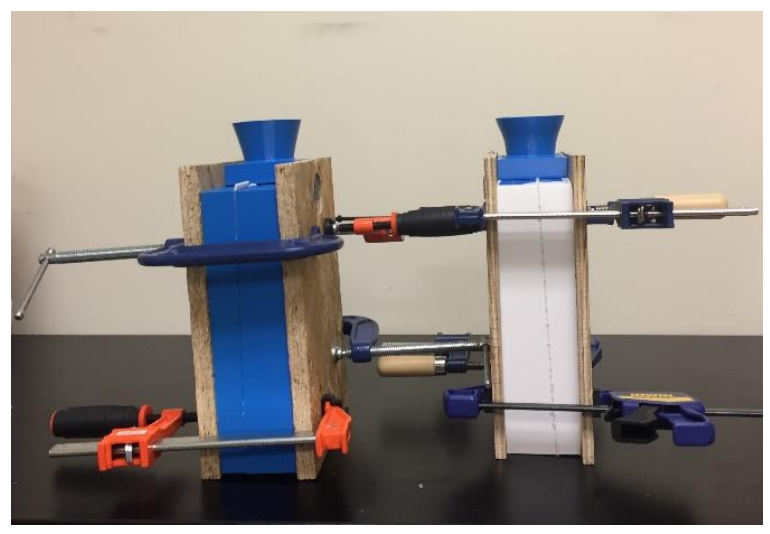

Figure 31: Assembled molds ready for casting right and left surrogate hands.

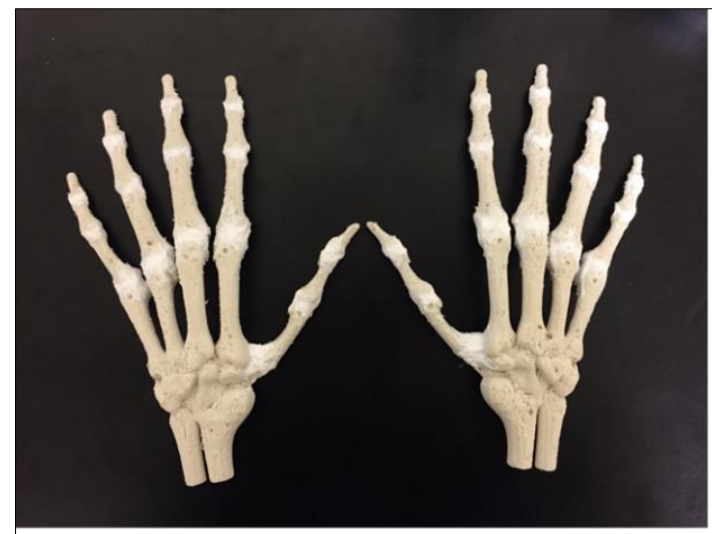

a

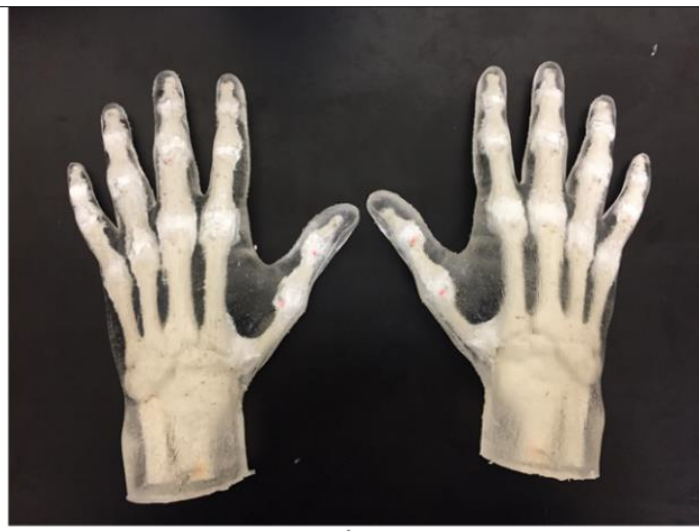

b

Figure 32: (a) The final design of the finished bone structure ( $3^{\text {rd }}$ generation). (b) The casted right and left Gel \#4.3 surrogate hands. 


\subsubsection{Experimental design}

Using the $1^{\text {st }}$ generation of bone structure, the procedure described in Section 4.2.1.3.2 was applied for creating surrogate hands using Gelatin grades \#0, \#2, \#3, and \#4 (see Table 11). The surrogate hands were impact tested and the performance data were compared to data obtained from the cadaveric hand tests performed during Aim \#1. Using the gel grade that performed the best (Gelatin \#4), an iterative adjustment process was performed to improve the global stiffness of the surrogate hand. The different generations of surrogate hands were impact tested using 4 specimens of Gel \#4.1, 4 specimens of Gel \#4.2, and 5 specimens of Gel \#4.3 surrogate hands.

The impact tests were performed on each proximal interphalangeal (PIP) joint (including thumb interphalangeal IP joint), on each metacarpophalangeal (MCP) joint, and the middle point of each metacarpal bone, for a total of 15 impacts on each surrogate hand (Figure 33). Prior to impact testing, a thin latex glove was put on the surrogate hand to facilitate clear marking of the impact position. The latex gloves are very thin $(\sim 0.10$ to $\sim 0.15 \mathrm{~mm})$, and their effect was assumed to be negligible. The addition of the latex glove also served as a "skin" to reduce the hand surface friction while putting the metacarpal gloves on the surrogate hand.

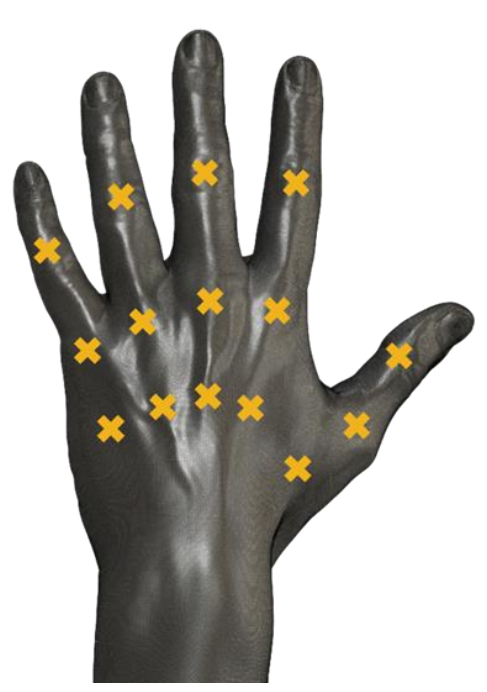

Figure 33: impact positions 


\subsubsection{Testing set-up description}

The impact testing set-up is the same as explained in Aim \#1 (Section 3.2.2).

\subsubsection{Testing procedure}

Surrogate hands were first inspected for any manufacturing defects or irregularities. The latex glove was then put on and impact locations were marked onto the glove dorsal region to maintain test consistency. Next, the hand was placed on the force plate in resting and nearly flat position and 15 impacts were then performed following a randomized sequence on the marked positions.

\subsubsection{Data processing}

The impactor bouncing behavior, measured by the coefficient of restitution (COR) and energy loss (EL), was used to carry out the comparison with the cadaveric hand results. The COR for vertically falling objects can be calculated from the ratio of the rebound height to the initial drop height (Figure 34) (Equation 2). The COR is often denoted by $e$ and explained as a parameter for energy loss (EL) due to objects collision (Equation 3) (Haron \& Ismail, 2012). Theoretically, COR values for perfectly plastic and perfectly elastic impacts are 0 and 1 , respectively. While the rebound height of the falling object in the former case is 0 , the rebound height of the falling object in the latter case is equal to the initial drop height. It is important to note that, since the impactor drop distance is small $(0.2 \mathrm{~m})$, the friction of the impacting bodies (steel impactor and surrogate hand) and the air drag are not considered in our calculations.

Previous studies indicated that air drag is negligible for drop of bodies from small height (Aryaei et al., 2010; Sandeep et al., 2020). Also, bodies friction and air drag could be neglected since the experimental conditions and surrounding environment are constant across all tests. 
The averages COR and EL from all impacted positions of cadaver hands tested during Aim \#1 were calculated and used as reference points. Mean COR and EL values for the surrogate hands with gel grades $0,2,3$, and 4 were compared to cadaver hands. The gel grade that provided closer COR and EL to reference points was selected for the final design of the surrogate hand.

$$
\begin{aligned}
& \text { Coefficient of Restitution }(C O R)=\sqrt{\frac{\text { rebound height }}{\text { initial drop height }}} \\
& \text { Energy loss Percentage }(E L)=100 \times\left(1-C_{O} R^{2}\right)
\end{aligned}
$$

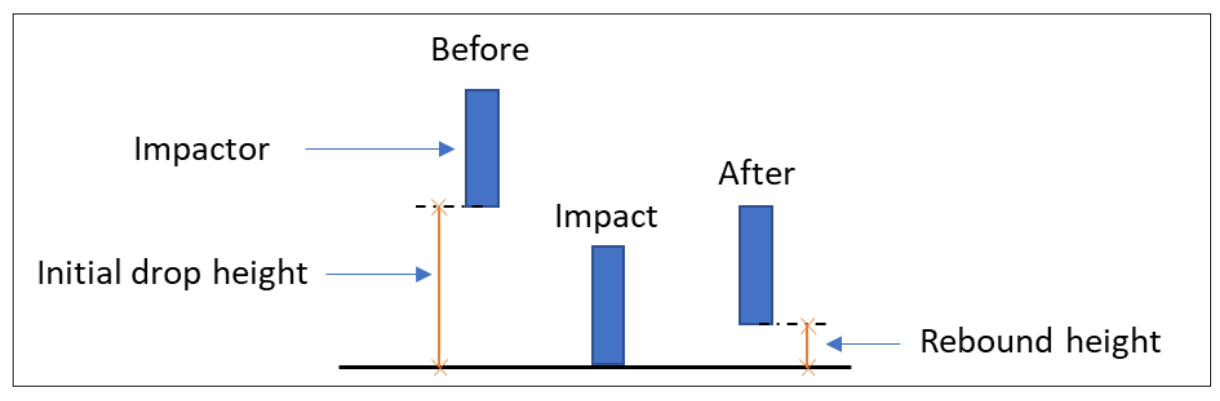

Figure 34: Schematic diagram for an impact initial drop height and rebound height for Coefficient of Restitution calculations.

After establishing the gel grade, the COR and the mean impact reaction forces at the three impacted positions (i.e. PIP, MCP, and Metacarpals) for the surrogate hand were compared with data from cadaver hand and guided the adjustment efforts. Further modifications were made to achieve COR and reaction forces comparable to that obtained from cadaver hands. A cutoff point of 1 standard deviation was used to compare the impact reaction forces with respect to the cadaver hand data. 


\subsubsection{Results}

The results of impact testing for all variations of surrogate hands are presented in this section. The initial testing including hands manufactured with Gelatins \#0, \#2, \#3 and \#4 revealed that the surrogate hand manufactured with Gelatin \#4 resulted in the closest COR and EL values (0.437 and 80.9, respectively) as compared to COR and EL of cadaver hands (0.380 and 85.6, respectively) (Figure 35). Thus, additional testing using four Gel \#4.1 surrogate hands was conducted in order to confirm this initial result and to evaluate impact response data. The average COR and EL of the 4 samples of Gel \#4.1 surrogate hands were 0.434 and 81.2, respectively, which confirmed the initial test (Figure 35). These values of COR and EL were respectively $14.2 \%$ and $-5 \%$ different from cadaver hand values. Therefore, Gelatin \#4 was selected to represent soft tissues in subsequent development of the surrogate hand.

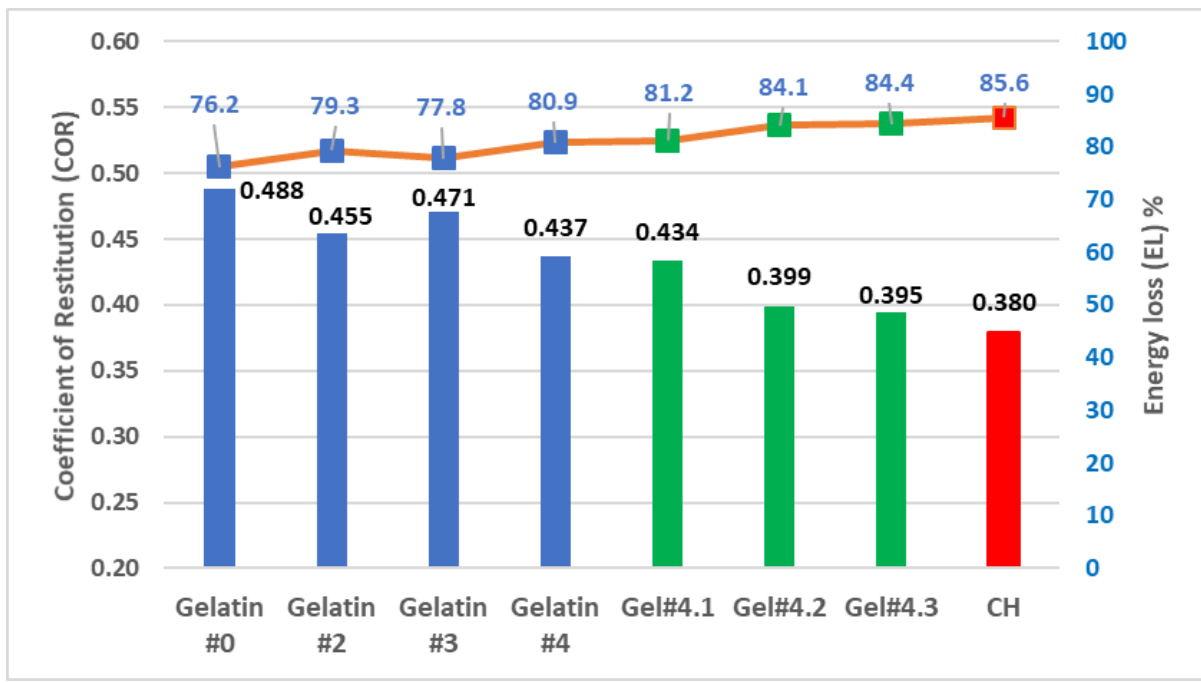

Figure 35: Coefficient of restitution (COR) and energy loss (EL) values for cadaver hands $(\mathrm{CH})$ and surrogate hands manufactured with different grades of synthatic gel and using different 3D printing settings.

Data shown in Table 14 summarizes the impact reaction forces obtained from cadaver hands and all generations of Gel \#4 surrogate hands. The calculated PRF differences between Gel \#4.1 and cadaver hands showed that PRF at the Metacarpal position of Gel \#4.1 was very close to that 
of cadaver hands (-1\% difference). On the other hand, the difference in PRF values at the PIP and MCP positions were $45 \%$ and $74 \%$, respectively. Therefore, further modifications to the 3D printing settings were performed to achieve more comparable impact reaction forces.

Table 14: The percentage mean difference in peak reaction force $(\% \Delta \mathrm{PRF})$ between cadaver hands $(\mathrm{CH})$ and different generations of Gel \#4 surrogate hands. Difference columns compare former column to cadaver hand column. Bolded values are within 1 standard deviation of cadaver hand values.

\begin{tabular}{c|ccc|cc|cc|cccc}
\hline \multicolumn{11}{c}{ PRF $(\mathrm{N})$} \\
\cline { 2 - 12 } Position & \multirow{2}{*}{ CH } & SD & $\begin{array}{c}\text { COV } \\
{[\%]}\end{array}$ & $\begin{array}{c}\text { Gel } \\
\# 4.1\end{array}$ & $\begin{array}{c}\% \Delta \\
\text { PRF }\end{array}$ & $\begin{array}{c}\text { Gel } \\
\# 4.2\end{array}$ & $\begin{array}{c}\% \Delta \\
\text { PRF }\end{array}$ & $\begin{array}{c}\text { Gel } \\
\# 4.3\end{array}$ & $\begin{array}{c}\% \Delta \\
\text { PRF }\end{array}$ & \multirow{2}{*}{ SD } & $\begin{array}{c}\text { COV } \\
{[\%]}\end{array}$ \\
\hline PIP & 2,468 & 288 & 12 & 3,576 & 45 & $\mathbf{2 , 5 9 3}$ & 5 & $\mathbf{2 , 6 3 2}$ & 7 & 105 & 4 \\
MCP & 1,799 & 287 & 16 & 3,132 & 74 & 1,423 & -21 & $\mathbf{1 , 7 9 8}$ & 0 & 276 & 15 \\
Metacarpals & 1,640 & 316 & 19 & $\mathbf{1 , 6 2 9}$ & -1 & 1,065 & -35 & $\mathbf{1 , 4 5 2}$ & -11 & 149 & 10 \\
\hline
\end{tabular}

Results obtained from Gel \#4.2 surrogate hands showed improvements in COR, EL, and impact reaction forces. The COR and EL values for this generation were 0.399 and 84.1, respectively (Figure 35). These values of COR and EL were 5\% and -2\% different from cadaver hand values which illustrate improvement in bouncing behavior and global stiffness. In terms of the impact reaction forces, the greatest improvement was at the PIP position which resulted in an average value only $5 \%$ higher than the reference point (Table 14$)$. This value $(2,593 \mathrm{~N})$ is within 1 standard deviation of cadaver hand data $(2,468 \mathrm{~N})$. Also, there was an improvement on PRF at the MCP position from being 74\% higher than reference point (in Gel \#4.1 generation) to $21 \%$ less than the reference point. However, the PRF value at MCP position $(1,423 \mathrm{~N})$ was still not within 1 standard deviation of cadaver hand data $(1,799 \mathrm{~N})$. On the other hand, average Metacarpals PRF value was $23 \%$ less than reference point, which is worse than the value obtained from Gel \#4.1 surrogate hand (3\% less than reference point).

The changes implemented in the surrogate hand Gel \#4.3 resulted into COR value of 0.395 and EL value of 84.4 (Figure 35). These values of COR and EL are almost the same as cadaver hands values with only $4 \%$ and $-1 \%$ difference, respectively. On the other hand, the PRF values 
at PIP, MCP, and Metacarpals improved with only 7\%, 0\%, and $-11 \%$ differences from cadaver hands values, respectively (Table 14). Also, the PRF values at all positions were within 1 standard deviation of cadaver hand data. Finally, the smaller coefficient of variation (COV) values for Gel \#4.3 surrogate hands compared to cadaver hands (Table 14) illustrates the reduced variability in the surrogate hands, which is anticipated to improve results accuracy.

Based on the improvement in the PRF values as well as the COR and EL values, the surrogate hand Gel \#4.3 provided a comparable global stiffness to cadaver hands tested. Therefore, this design was utilized during the second part of this study which involved testing the impact resistance of selected metacarpal gloves.

\subsection{Objective 2: Glove impact resistance using surrogate hand}

The second objective of Aim \#2 examined the protection levels provided by three commonly used metacarpal gloves under impact loads using surrogate hand Gel \#4.3.

\subsubsection{Experimental design}

Impact tests were performed on surrogate hands with and without gloves using the positions explained in Section 4.2 .2 (i.e. 15 impacts on each hand). Three types of metacarpal gloves were evaluated during this experiment (Figure 36). Specifications and initial impact performance evaluation of the selected gloves are explained below in Section 4.3.2.

In this portion of the study, each glove type was tested under impact loads using a set of five surrogate hands. To facilitate with-glove vs. no-glove comparisons, results from a set of five surrogate hands tested without gloves were used as baseline data. This design resulted in manufacturing and testing a total of 20 surrogate hands. Pictures included in Appendix D.1 illustrate the different set of specimens with and without gloves used for the tests. To ensure 
consistency and to track the manufacturing quality, weight, length (from wrist crease to tip of middle finger), breadth (at knuckles level), and circumference (at knuckles level) of each surrogate hand were recorded prior to testing (see pictures in Appendix D.2). The recorded measurements and the reference data of male $50^{\text {th }}$ percentile measurements are shown in Table 15 and Table 16. Overall, all measurements of all manufactured surrogate hands were similar which is illustrated by the small standard deviation and COV values in Table 14 . The slight fluctuation in the measurements was primarily attributed to operator error.

Table 15: Weight, length, breadth, and circumference of all tested surrogate hands with no-glove and with all types of considered gloves.

\begin{tabular}{|c|c|c|c|c|c|}
\hline ID & Weight [g] & $\begin{array}{l}\text { Length } \\
{[\mathrm{cm}]}\end{array}$ & $\begin{array}{c}\text { Breadth } \\
{[\mathrm{cm}]}\end{array}$ & $\begin{array}{c}\text { circumference } \\
{[\mathrm{cm}]}\end{array}$ & Glove size \\
\hline No-glove-1 & 462.2 & 19.3 & 8.9 & 21.4 & \multirow{5}{*}{ - } \\
\hline No-glove-2 & 455.4 & 19.4 & 8.8 & 21.5 & \\
\hline No-glove-3 & 459.7 & 19.4 & 9 & 21.3 & \\
\hline No-glove-4 & 458.3 & 19.3 & 8.9 & 21.5 & \\
\hline No-glove-5 & 453.9 & 19.4 & 9 & 21.3 & \\
\hline Avg. & 457.9 & 19.4 & 8.9 & 21.4 & - \\
\hline G1-1 & 466.4 & 19.4 & 9.0 & 21.4 & \multirow{5}{*}{ XL } \\
\hline G1-2 & 460.8 & 19.4 & 8.8 & 21.5 & \\
\hline G1-3 & 454.7 & 19.3 & 8.9 & 21.4 & \\
\hline G1-4 & 458.2 & 19.4 & 8.8 & 21.4 & \\
\hline G1-5 & 456.8 & 19.4 & 8.9 & 21.5 & \\
\hline Avg. & 459.4 & 19.4 & 8.9 & 21.4 & - \\
\hline G2-1 & 456.8 & 19.4 & 8.8 & 21.3 & \multirow{5}{*}{$\mathrm{L}$} \\
\hline G2-2 & 457.5 & 19.3 & 8.9 & 21.4 & \\
\hline G2-3 & 454.1 & 19.3 & 8.8 & 21.4 & \\
\hline G2-4 & 455.1 & 19.4 & 8.9 & 21.5 & \\
\hline G2-5 & 458.7 & 19.4 & 9.0 & 21.4 & \\
\hline Avg. & 456.4 & 19.4 & 8.9 & 21.4 & - \\
\hline G3-1 & 456.7 & 19.5 & 9.0 & 21.5 & \multirow{5}{*}{ XL } \\
\hline G3-2 & 456.3 & 19.4 & 9.0 & 21.4 & \\
\hline G3-3 & 464.1 & 19.4 & 8.9 & 21.3 & \\
\hline G3-4 & 457.6 & 19.5 & 9.0 & 21.5 & \\
\hline G3-5 & 452.9 & 19.3 & 8.8 & 21.5 & \\
\hline Avg. & 457.5 & 19.4 & 8.9 & 21.4 & - \\
\hline Grand Avg. & 457.8 & 19.4 & 8.9 & 21.4 & - \\
\hline SD & 3.5 & 0.1 & 0.1 & 0.1 & - \\
\hline $\mathrm{COV}$ & $0.8 \%$ & $0.3 \%$ & $0.9 \%$ & $0.4 \%$ & - \\
\hline
\end{tabular}


Table 16: Length, breadth, and circumference of $50^{\text {th }}$ percentile male hand (obtained from literature).

\begin{tabular}{cccc}
\hline Reference & $\begin{array}{c}\text { Length } \\
{[\mathrm{cm}]}\end{array}$ & $\begin{array}{c}\text { Breadth } \\
{[\mathrm{cm}]}\end{array}$ & $\begin{array}{c}\text { Circumference } \\
{[\mathrm{cm}]}\end{array}$ \\
\hline Garrett, 1971 & 19.7 & 8.9 & 21.6 \\
Greiner, 1991 & 19.4 & 9.5 & 21.4 \\
Caesar & 20.1 & - & 21.1 \\
\hline Average & 19.7 & 9.2 & 21.4 \\
SD & 0.35 & 0.42 & 0.25 \\
\hline
\end{tabular}

\subsubsection{Gloves specifications and performance}

The three selected gloves are commercially available and often used in the mining industry. Two of these gloves are same as the Aim \#1 study (i.e. G1 and G2) to facilitate comparison with cadaveric hand data obtained during Aim \#1 (Figure 36). The third glove (G3) considered in this study is a pigskin leather-based glove advertised to be suitable for mining activities (Figure 36). The three types of metacarpal gloves selected for this study were considered to have different levels of protection based on their designs, as well as different placements and quantity of thermoplastic rubber (TPR). The sizes of the gloves used in the test are shown in Table 15. The major factor for selecting a glove size was the ability to insert the glove on the surrogate hands.

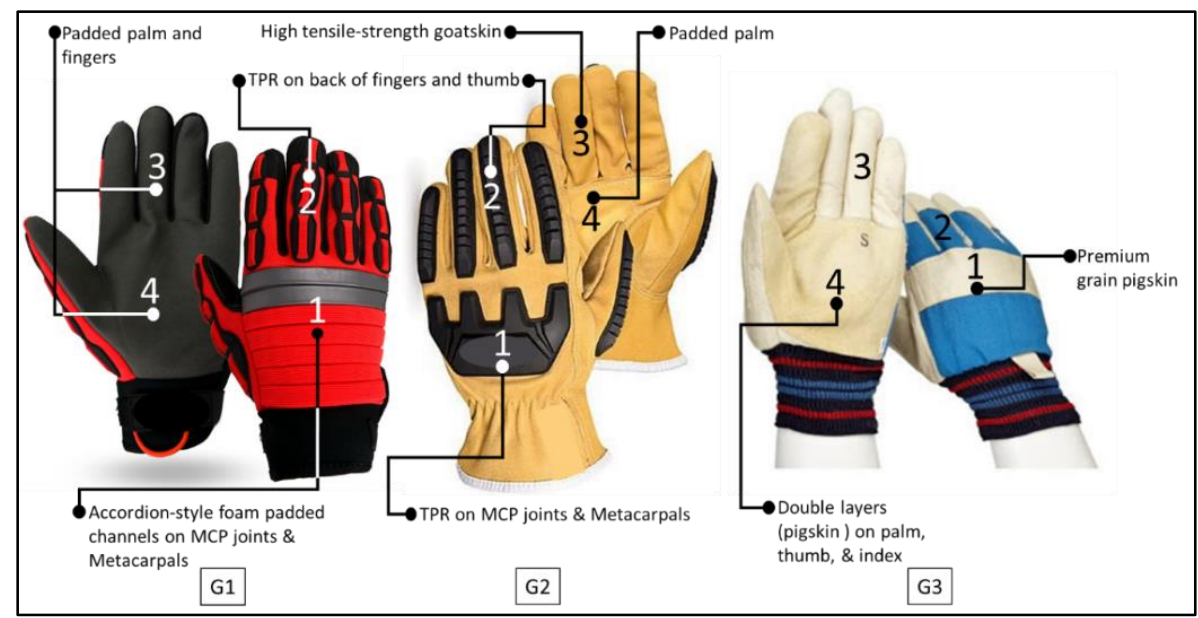

Figure 36: Metacarpal gloves considered in this study. Numbers correspond to the regions where types of material were evaluated (see Table 17) and thickness measurements were performed (see Table 18). 
Glove G1 includes TPR reinforcements only in the finger region and foam padding (96\% Polyester and 4\% Spandex) on MCP joints and back of hand (metacarpal bones) region. The palmar (anterior) side of G1 is composed of a foam pad layer (50\% nylon and $50 \%$ polyurethane). Glove G2 includes TPR reinforcements on the fingers, MCP joints, and the metacarpals region. Each of the anterior and posterior sides is composed of an external goatskin layer and an internal Kevlar lining layer. The palmar region of G2 is also supported with gel pads. Glove G3 is comprised of an external pigskin layer in the palm and knuckle areas with double layers on palm, thumb, and index fingers. Each of the anterior and posterior sides is composed of polyester fabric and inner cotton lining layers. The posterior side of G3 is also reinforced with a foam pad layer. Table 17 summarizes the materials and layers that constitute the glove regions identified in Figure 36. Data sheets of the selected gloves are provided in Appendix C. Appendix D.4 includes pictures of the disassembled gloves showing the different material layers.

Table 17: Layers and material types for the tested gloves at different regions. Region numbers are illustrated in Figure 36.

\begin{tabular}{|c|c|c|c|c|}
\hline & \multicolumn{4}{|c|}{ Layers and material types } \\
\hline Side & \multicolumn{2}{|c|}{ Posterior } & \multicolumn{2}{|c|}{ Anterior } \\
\hline Region & 1 & 2 & 3 & 4 \\
\hline G1 & $\begin{array}{l}\text {-Foam padded channels } \\
\text { (96\% Polyester; } 4 \% \\
\text { Spandex) }\end{array}$ & $\begin{array}{l}\text {-TPR } \\
\text {-Polyester fabric layer }\end{array}$ & $\begin{array}{l}\text {-Foam pad }(50 \% \\
\text { nylon \& } 50 \% \\
\text { polyurethane) }\end{array}$ & $\begin{array}{l}\text {-Foam pad (50\% } \\
\text { nylon \& 50\% } \\
\text { polyurethane) }\end{array}$ \\
\hline $\mathrm{G} 2$ & $\begin{array}{l}\text {-TPR } \\
\text {-Goatskin layer } \\
\text { - Kevlar lining layer }\end{array}$ & $\begin{array}{l}\text {-TPR } \\
\text {-Goatskin layer } \\
\text { - Kevlar lining layer }\end{array}$ & $\begin{array}{l}\text {-Goatskin layer } \\
\text {-Kevlar lining } \\
\text { layer }\end{array}$ & $\begin{array}{l}\text {-Goatskin layer } \\
\text {-Kevlar lining } \\
\text { layer } \\
\text {-Gel pads }\end{array}$ \\
\hline G3 & $\begin{array}{l}\text {-Polyester fabric layer } \\
\text { (blue) } \\
\text {-Foam pad } \\
\text {-Cotton lining layer }\end{array}$ & $\begin{array}{l}\text {-Pigskin layer } \\
\text {-Polyester fabric layer } \\
\text { (blue) } \\
\text {-Foam pad } \\
\text {-Cotton lining layer }\end{array}$ & $\begin{array}{l}\text {-Pigskin layer } \\
\text {-Cotton lining } \\
\text { layer }\end{array}$ & $\begin{array}{l}\text {-Double pigskin } \\
\text { layers } \\
\text {-Cotton lining } \\
\text { layer }\end{array}$ \\
\hline
\end{tabular}


Additionally, one glove of each brand was disassembled to measure thicknesses of layers. Data shown in Table 18 summarizes the mean thicknesses of the selected gloves at different regions. For each glove different regions were measured independently after cutting the glove into two parts (i.e. posterior and anterior). The measurements were performed using a high precision digital caliper.

Table 18: Means of material layers thicknesses for the tested gloves at different regions. Region numbers are illustrated in Figure 36.

\begin{tabular}{c|c|c|c|c}
\hline & \multicolumn{4}{|c}{ Thickness [mm] } \\
\hline Side & \multicolumn{2}{|c|}{ Posterior } & 3 & 4 \\
\hline Region & 1 & 2 & 1.3 & 1.3 \\
\hline G1 & 4.8 & $6.6(\mathrm{TPR}=4.1)$ & 2 & 6.5 \\
\hline G2 & $6.7(\mathrm{TPR}=4.7)$ & $8.5(\mathrm{TPR}=6.6)$ & 1.0 & 2.1 \\
\hline G3 & 5.0 & 3.8 & &
\end{tabular}

To further evaluate the protection performance of the selected gloves, one pair of each brand was impacted directly without using a surrogate hand on the same 15 impact positions. This test was performed first on a full glove and then on only the posterior part of the glove (i.e. dorsal part), after cutting out the anterior part (i.e. palmar part). The main purpose of this test was to generate additional baseline data to further improve the interpretation of the study findings. Mean PRF values for each condition and the percentage differences between full glove and dorsal side tests are shown in Figure 37. PRF values for glove G1 from full glove and dorsal side tests were only $-2 \%$ different from each other. On the other hand, the percentage differences in PRF between full glove and dorsal side tests for gloves G2 and G3 were -8\% and -6\% respectively. Such differences could be attributed to the type of materials and number of layers used to reinforce the palmar side of gloves. 
Figure 38 compares PRF values between different gloves when impacted without hand.

Within the full glove test, mean PRF values for gloves G1 and G3 were similar (2\% difference), and they differ from glove $\mathrm{G} 2$ by $7 \%$ and $-6 \%$, respectively. On the other hand, the mean PRF values for glove G3 at the dorsal side condition were only $2 \%$ and $-4 \%$ smaller than that of G1 and G3, respectively.

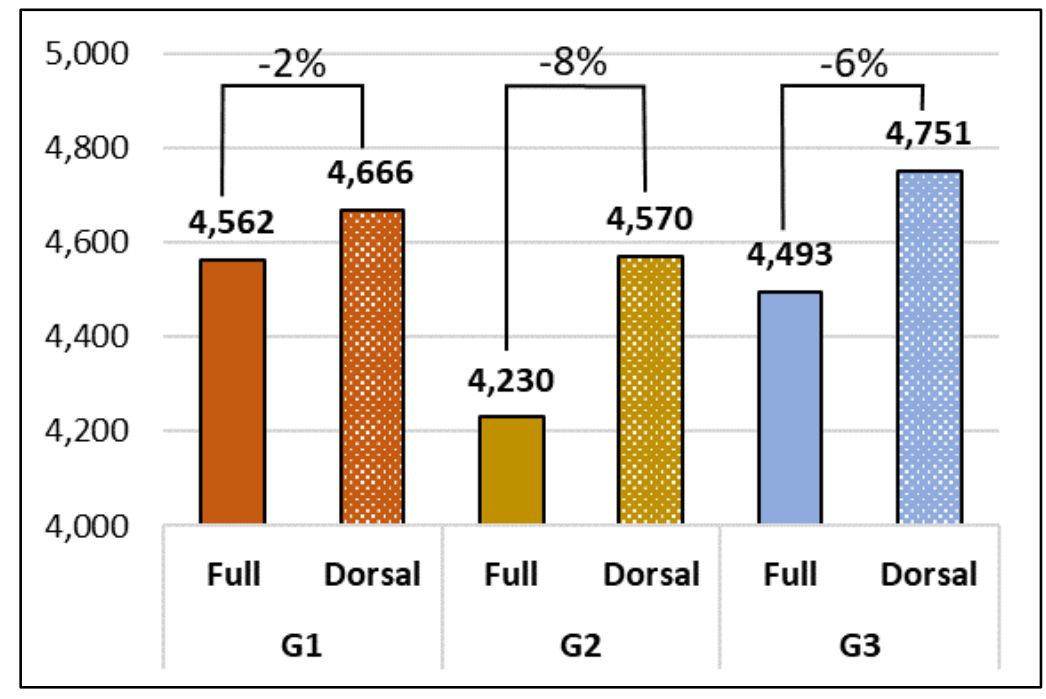

Figure 37: Comparisons of PRF values between full glove and only dorsal part of glove impacted directly without hands.

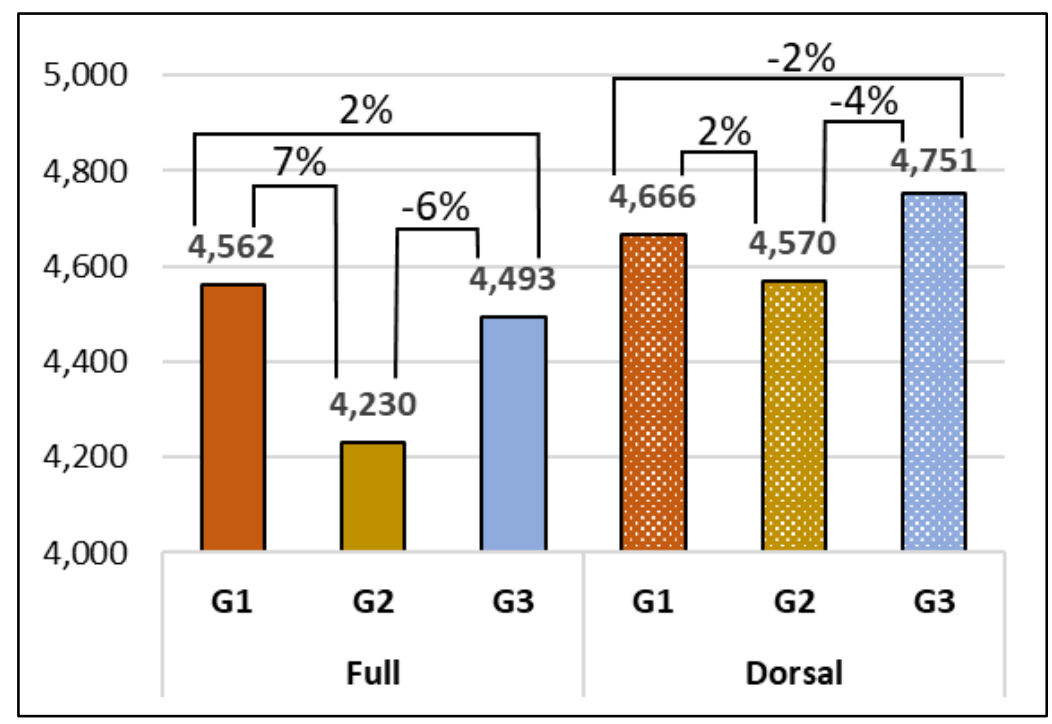

Figure 38: Comparisons of PRF values between conditions of gloves impacted without hand. 


\subsubsection{Testing set-up description}

Same as Aim \#1 (see Section 3.2.2)

\subsubsection{Testing procedure}

Same as explained in Section 4.2.4

For the tests with metacarpal gloves, the glove was put on a surrogate hand and the impact positions were marked onto the dorsal region to ensure impact onto the targeted position. Also, the gloved hands were positioned to ensure perpendicular impact on the targeted zone of protection.

\subsubsection{Data processing}

\subsubsection{Impact forces}

Descriptive analysis was performed to compare the mean PRF across the gloves (with and without) and the impact regions. Furthermore, the Impact Protection Index (IPI) was used to quantify the amount of protection for each glove. This analysis methodology is similar to the Aim \#1 study presented in detail in Section 3.2.4.1.

\subsubsection{Fracture evaluation}

After completion of all impacts, the synthetic gel was removed from the surrogate hands and the bone structures were examined to identify and count the number of fractures. Visible damage to the bone structure was considered as a fracture regardless of the damage severity. Similar to the Aim \#1 study (see Section 3.2.4.3), overall number of fractures (injurious impacts) in each region was normalized to the total number of impacts in that region. The resulting values were used as a secondary protection measure for the tested gloves and the no-glove condition. 


\subsubsection{Statistical analysis}

Prior to the statistical analysis, ANOVA assumptions were tested and verified (Montgomery, 2012) (see Appendix A for details). Two-way ANOVA test was conducted to test the effect of Protection and Position on PRF values. The independent variable "Protection" was treated at four levels: the no-glove level, and one level for each type of glove (i.e. G1, G2, and G3 levels). The "no-glove" level included the five surrogate hands tested without a glove. The variable "Position" was treated at three levels: PIP joint, MCP joint, and Metacarpal bones. Additionally, student's t-test analysis was performed to compare the mean PRF values between the levels of significant variables. A criterion $p$-value of $\leq 0.05$ was used in all statistical analyses, which were performed in JMP Version 14 (SAS Institute Inc., Cary, NC). Descriptive analysis was used to compare number of fractures as explained in Section 4.3.4.2.

\subsubsection{Results}

\subsubsection{Impact force evaluation}

A total of 300 controlled impacts were performed in the present study: 75 impacts for each condition (i.e. no-glove, G1, G2, and G3). The results of ANOVA tests showed that the effects of the independent variables Protection (Table 19) and Position (Table 20) on the mean values of PRF were statistically significant ( $\mathrm{P}<0.001$ ) (see Appendix B for ANOVA detailed tables). The interaction effect of the two independent variables was statistically not significant $(\mathrm{P}=0.5)$. Data shown in Figure 39 demonstrates the results of the student's t-test on the differences between the levels of the variable Protection. Mean PRF values of the levels G1, G2, and G3 were statistically different from the no-glove level $(\mathrm{P}<0.001)$. Also, the results showed a significant difference on the mean PRF values between the levels G1 and G2 (P<0.001), and the levels G1 
and G3 (P=0.0017). On the other hand, PRF values of the levels G2 and G3 were not statistically different $(\mathrm{P}=0.2976)$.

Table 19: Results of ANOVA test for the effect of Protection on PRF. Bold P-value indicates a statistically significant difference.

\begin{tabular}{c|cccc|c}
\hline Protection & no-glove & G1 & G2 & G3 & $P$-value \\
\hline Average PRF (SD) [N] & $1,960(542)$ & $1,697(554)$ & $1,428(591)$ & $1,493(523)$ & $<0001$ \\
\hline
\end{tabular}

Table 20: Results of ANOVA test for the effect of Position on PRF. Bold P-value indicates a statistically significant difference.

\begin{tabular}{c|ccc|c}
\hline Position & PIP & MCP & Metacarpals & $P$-value \\
\hline Average PRF $(\mathrm{SD})[\mathrm{N}]$ & $2,357(235)$ & $1,386(311)$ & $1,190(252)$ & $<\mathbf{0 . 0 0 1}$ \\
\hline
\end{tabular}

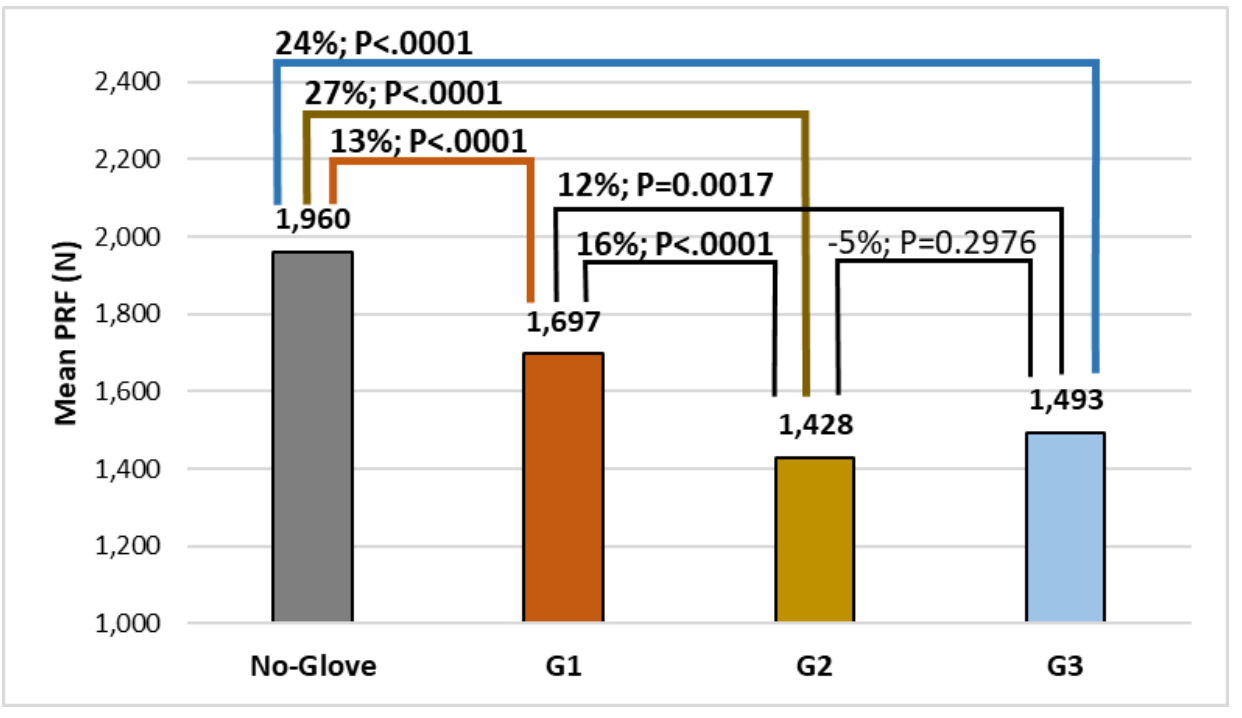

Figure 39: Mean values of PRF and results of the students' t-test for the effect of Protection on PRF at different levels of the independent variable Protection. A bold P-value indicates a statistically significant difference.

Results summarized in Table 21 show the mean PRF, TPRF, and kinetic energy (KE) for all levels of the variable Protection. The percentage mean difference in PRF ( $\% \Delta$ PRF) between each level tested with glove at each Position and the no-glove level are also shown in Table 21. 
$\% \triangle$ PRF between glove G1 and the "no-glove" condition at the levels: PIP, MCP, and Metacarpals were $9 \%, 21 \%$, and $12 \%$, respectively. For glove G 2 the $\% \Delta$ PRF were: $16 \%$ for PIP joints, 37\% for MCP joints, and 36\% for Metacarpals. For glove G3, the $\% \Delta$ PRF at the levels: PIP, MCP, and Metacarpals were 17\%, 33\%, and 25\%, respectively. The IPI values calculated during this study for the gloves G1, G2, and G3 were 27,40 , and 35, respectively (Table 22). This table also includes IPI values calculated during Aim\# 1 study and from the study of Sosa et al. (2019) for the same evaluated gloves. The average KE for the combination of all glove types and for the no-glove level were $6.8 \mathrm{~J}$ and $7.1 \mathrm{~J}$, respectively.

Table 21: Summary of average PRF, TPRF, and KE values; and PRF values change between the level "no-glove" and the evaluated gloves across all levels of Protection and Position.

\begin{tabular}{c|l|ccc|c|c}
\hline \multirow{3}{*}{ Protection } & Position & $\begin{array}{c}\mathrm{PRF} \\
{[\mathrm{N}]}\end{array}$ & $\begin{array}{c}\mathrm{TPRF} \\
{[\mathrm{N}]}\end{array}$ & $\begin{array}{c}\mathrm{KE} \\
{[\mathrm{J}]}\end{array}$ & $\begin{array}{c}\mathrm{PRF}_{(\mathrm{NG})}- \\
\mathrm{PRF}_{(\mathrm{G})} \\
{[\% \Delta \mathrm{PRF}]}\end{array}$ & $\begin{array}{c}\mathrm{PRF}_{(\mathrm{NG})}-\mathrm{PRF}_{(\mathrm{G})} \\
{[\% \Delta \mathrm{PRF}]}\end{array}$ \\
\hline \multirow{3}{*}{ no-glove } & PIP & 2,632 & 2,181 & 7.5 & - & - \\
& MCP & 1,798 & 1,458 & 7.1 & - & - \\
& Metacarpals & 1,452 & 1,198 & 6.6 & - & - \\
\hline \multirow{3}{*}{ G1 } & PIP & 2,392 & 1,980 & 7.6 & 9 & -3 \\
& MCP & 1,417 & 1,216 & 6.9 & 21 & 10 \\
& Metacarpals & 1,284 & 1,082 & 6.5 & 12 & 13 \\
\hline \multirow{3}{*}{ G2 } & PIP & 2,224 & 1,782 & 7.3 & 16 & 23 \\
& MCP & 1,128 & 893 & 6.3 & 37 & 4 \\
& Metacarpals & 934 & 805 & 6.0 & 36 & - \\
\hline \multirow{3}{*}{ G3 } & PIP & 2,183 & 1,830 & 7.5 & 17 & - \\
& MCP & 1,204 & 1,080 & 6.8 & 33 & 25 \\
\hline
\end{tabular}

Note: * corresponds to cadaveric hand data from Aim \#1 study. (obtained from Table 7).

Table 22: Values of IPI compared to Aim \#1 study and previously reported data.

\begin{tabular}{c|c|c|c|c}
\hline Glove & IPI [\%] & IPI* [\%] & IPI** [\%] & IPI*** [\%] \\
\hline G1 & 27 & 10 & 40 & 35 \\
G2 & 40 & 23 & 51 & 53 \\
G3 & 35 & - & 37 & 27 \\
\hline
\end{tabular}

Note: IPI* is from Aim \#1 study. IPI** and IPI*** are from the study of Sosa et al. (2019) for semi-flexible and semi-rigid surrogate hands, respectively. 


\subsubsection{Fracture evaluation}

A total of 300 impacts produced a total of 132 fractures across all Protection levels. The distribution of fractures per Protection level is shown in Figure 40(A). In this graph, 44\% of the fractures were at the no-glove condition, $25 \%$ at the $\mathrm{G} 1$ condition, $6 \%$ at the $\mathrm{G} 2$ condition, and 25\% at the G3 condition. In order to facilitate comparisons with Aim \#1 results, the distribution of injurious impacts after excluding glove G3 data is shown in Figure 40(B). For this scenario, a total of 225 controlled impacts produced a total of 99 fractures. The distribution of fractures between the Protection levels: no-glove, G1, and G2 were 59\%, 33\%, and 8\%, respectively. The distribution of injurious impacts produced in the cadaveric hands (Aim \#1) is shown in Figure 40(C). Note that the sample sizes for Aim \#1 and Aim \#2 were not equal which may cause unbalanced comparisons. For Aim \#1, seven cadaveric hands were impacted without glove, and each glove was tested using three cadaveric hands. On the other hand, each Protection condition in Aim \#2 was tested using five surrogate hands. Therefore, number of fractures were normalized according to number of impacts to create the proportions of injurious impacts for each condition

(Figure 41 and Figure 42).

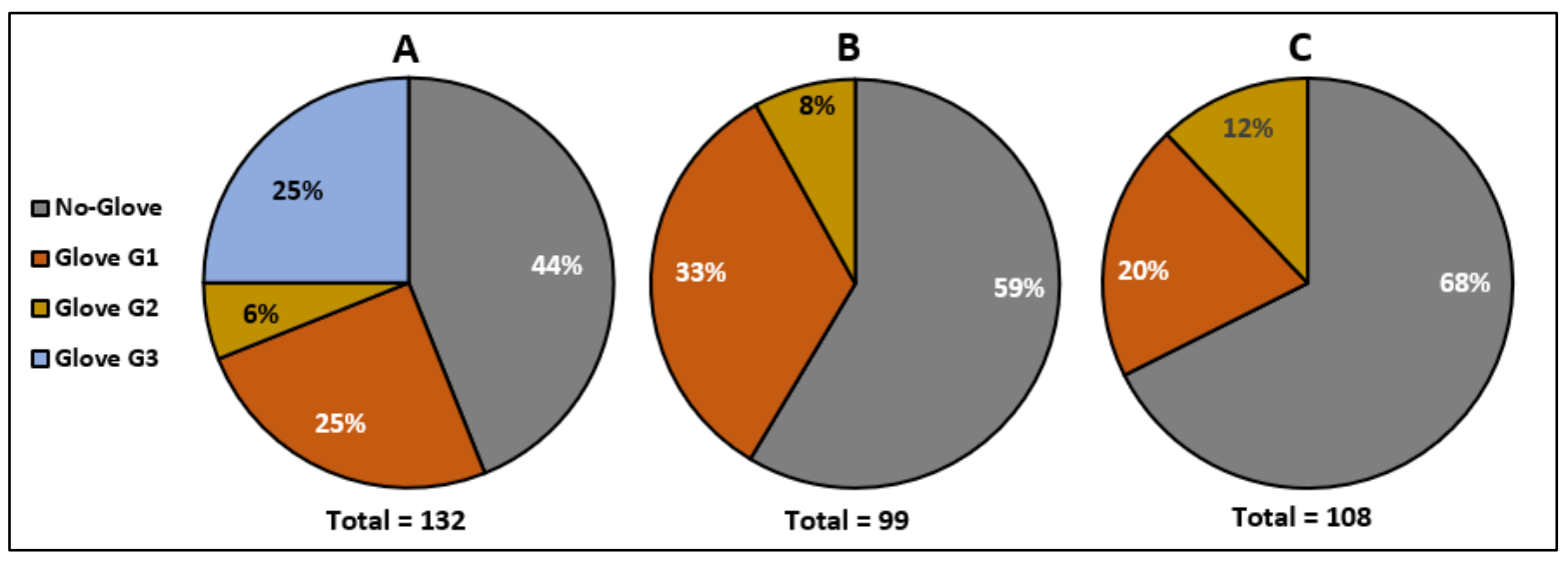

Figure 40: (A) Distribution of injurious impacts for each Protection condition. (B) Distribution of injurious impacts after excluding G3 data to facilitate comparisons with Aim \#1 results. (C) Distribution of injurious impacts in cadaveric hands (Aim \#1). 
Data shown in Figure 41 summarizes the overall proportions of injurious impacts in relation to the total number of impacts for all Positions combined and at each Position level for the noglove and with-glove conditions. The with-glove condition combines G1, G2, and G3 data. For the no-glove condition for Gel \#4.3 surrogate hands (Figure 41(a)), the proportions of fractures at PIP, MCP, and Metacarpal levels were 92\%, 52\%, and 88\%, respectively. On the other hand, the with-glove condition showed a reduction in the proportions of injurious impacts to $55 \%$ at the PIP joints, 24\% at the MCP joints, and 20\% at the Metacarpal level. When all Positions were combined, the proportion of injurious impacts reduced from $77 \%$ at the no-glove condition to $33 \%$ at the with-glove condition (i.e. $57 \%$ reduction).

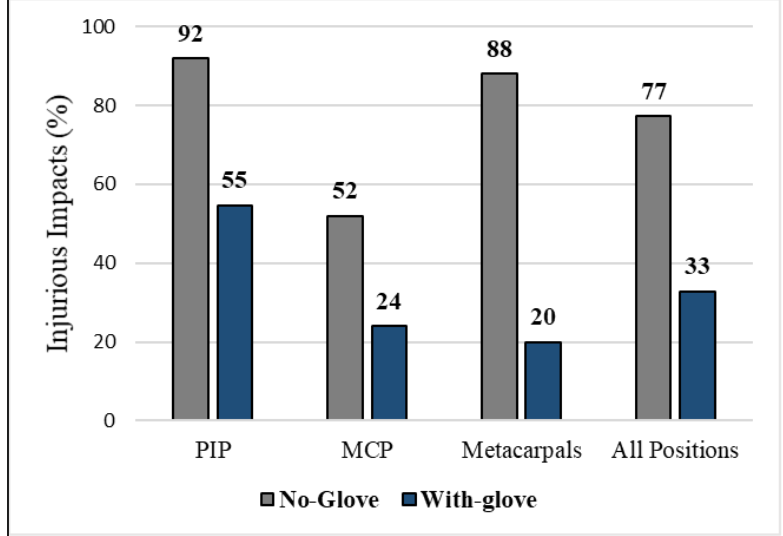

(a) Gel\#4.3 surrogate hands

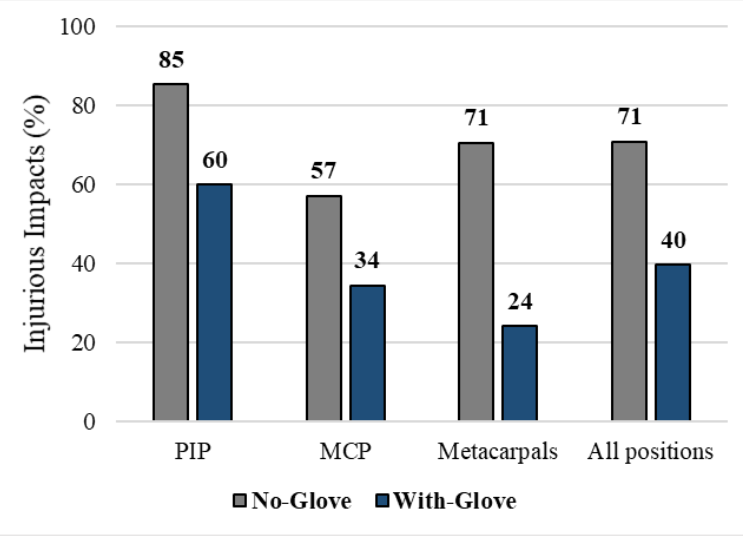

(b) Cadaveric hands (Aim \#1)

Figure 41: Percentage of impacts that resulted into a fracture (injurious impacts) per Position for with-glove (all gloves were combined) and no-glove Protection conditions. Percentages were calculated from the total number of impacts in each Position for each Protection condition. (a) For Gel \#4.3 surrogate hands data (Aim \#2); (b) For cadaveric hands data (Aim \#1).

However, as expected, the reduction of injurious impacts was not the same for all types of gloves (Figure 42). Considering all Positions, $77 \%$ of the impacts on surrogate hands with noglove were injurious while $44 \%, 11 \%$, and $44 \%$ of the impacts on gloves G1, G2, and G3 were injurious, respectively. Also, for glove G1, when evaluated by region of impact, $72 \%$ of the 
impacts on the PIP joints, $32 \%$ on MCP joints, and 28\% on the Metacarpals, were injurious.

Within glove G2, the percentages of injurious impacts on PIP, MCP, and Metacarpal levels were $16 \%, 8 \%$, and $8 \%$, respectively. For glove G3, $76 \%, 32 \%$, and $24 \%$ of the impacts on the PIP, MCP, and Metacarpal levels were injurious, respectively.

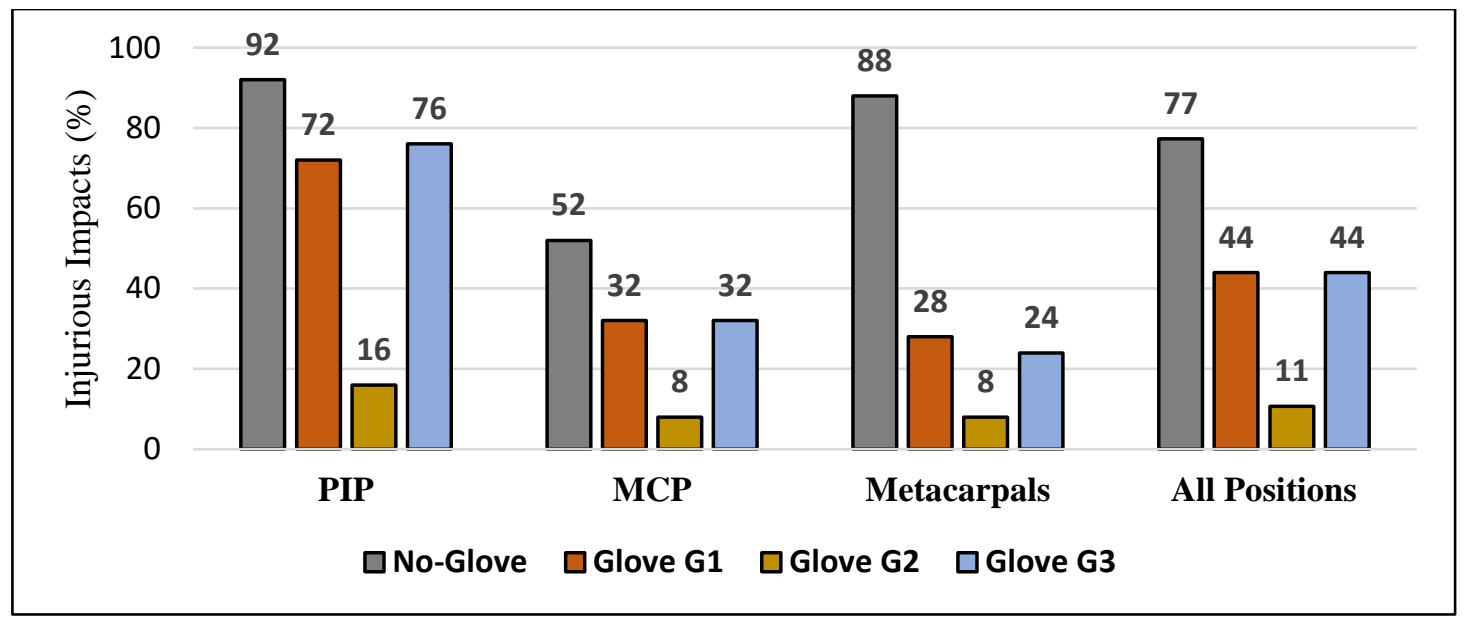

Figure 42: Percentage of impacts that resulted into a fracture (injurious impacts) for each Position and Protection condition. Percentages were calculated from the total number of impacts in each Position for each Protection condition.

\subsubsection{Discussion}

Three-dimensional (3D) printing and casting manufacturing techniques were used to develop surrogate hand specimens with dimensions corresponding to $50^{\text {th }}$ percentile male hand. $3 \mathrm{D}$ printed models of hand bones and medical-grade synthetic gel, representing the surrounding soft tissues, were utilized to replicate the overall biomechanical properties of the human hand. The surrogate hand specimens were validated using the impact response data from the cadaveric hand specimens presented in Chapter 3. The adjustment of stiffness of the surrogate hand followed an iterative development process in which each iteration was tested under impact and compared to data from the cadaveric hand specimens. The improvement process involved testing 4 grades of medical-grade synthetic gel and several combinations of 3D printing settings. The comparisons 
were carried out in terms of the peak rection force (PRF) values at different Positions and the global coefficient of restitution (COR) value.

Mean PRF values from the last generation of surrogate hands (Gel \#4.3) were within 1 standard deviation of cadaveric data, and their mean COR value was only $4 \%$ different from cadaveric data. Based on these results, the global stiffness of the surrogate hand Gel \#4.3 was considered comparable to human hand stiffness, and thus was adopted to replace a real human hand in additional glove impact testing. The use of surrogate hands was intended to overcome some of the cadaveric hand's limitations such as the limited sample size and high variability seen in previous studies (Carpanen et al., 2019; Loshek, 2015) and also in the results of Aim \#1 study.

The consistency of the manufacturing procedure developed in this work was reflected in the quality of the manufactured surrogate hands whose uniformity was monitored by measuring hands' weight, length, breadth, and circumference (Table 15). The coefficient of variation (COV) values of all measurements were less than $1 \%$ which illustrates the consistency across all manufactured surrogate hands. Also, these measurements are similar to $50^{\text {th }}$ percentile male hand measurements available in the literature (Garrett, 1971; Greiner, 1991; Harrison \& Robinette, 2002), which was expected as the digital model of the surrogate hand was scaled according to these values (Table 9).

The levels of protection of three types of metacarpal gloves were evaluated under impact using the surrogate hands. Two of the tested gloves (G1 and G2) were similar to the gloves tested during Aim \#1. All three gloves were also evaluated previously in the work of Sosa et al. (2019) using semi-rigid and semi-flexible surrogate hands. In their study, the semi-rigid surrogate hand was “...manufactured from segments of oak dowel rods that were sized and assembled to create a hand shape similar to a human hand." Also, the diameter of the wooden segments was constant. 
On the other hand, the semi-flexible surrogate hand was “...comprised of a 3D printed bone structure and a medical grade ballistic gel representing the soft tissue of a typical large-size hand" (Sosa et al., 2019). In that study, the overall structure of the semi-flexible surrogate hand was simplified in terms of the dimensions, shape, and proprieties of the 3D printing material (i.e. PLA) and the synthetic gel (i.e. Gelatin \#0). In this regard, the surrogate hands developed in this research represent an evolution with respect to the models presented in Sosa et al. (2019). Moreover, for comparison purposes, the values of IPI reported by Sosa et al. (2019) for the same gloves tested in the current study were summarized in Table 22.

In the current study, a series of controlled impact tests were performed on unprotected and protected surrogate hands to measure the change in force values and proportions of injurious impacts. Mean PRF values and number of fractures from surrogate hands tested with each of the evaluated gloves were compared to data of surrogate hands tested without gloves. The testing set-up was similar to the Aim \#1 study with a targeted nominal energy of $10 \mathrm{~J}$. However, the calculated KE values showed some fluctuations across the tested levels with an average KE for all impact tests of $6.9 \mathrm{~J}$ (Table 21). Similar to the discussion presented for Aim \#1 study, the fluctuation was attributed to the variability in the surrogate hand depth at the different impacted Positions and the different thicknesses of glove layers (Table 18), which reduced the drop distance from the targeted nominal $0.2 \mathrm{~m}$ to an average of $0.185 \mathrm{~m}$. Also, part of the energy loss was attributed to the friction of the testing machine which was explained in Section 3.4.

Similar to the cadaveric hand study, results of the current study suggested that the use of glove dissipated some of the impact energy transferred to the hand. Mean PRF values from tests on surrogate hands wearing gloves G1, G2, and G3 were significantly less than tests with noglove by $13 \%, 27 \%$, and 24\%, respectively (Figure 39). A more detailed comparison within each 
of the tested gloves showed that the $\% \Delta$ PRF for each Position of glove G1 was less than $\% \Delta$ PRF for each Position of G2 and G3 (Table 21). This result is consistent with Aim \#1 findings (only for G1 and G2) except for the Metacarpals Position, which in Aim \#1 showed more reduction in PRF for G1 relative to $\mathrm{G} 2$. The $\% \triangle \mathrm{PRF}$ for $\mathrm{G} 2$ at Metacarpals obtained from Aim \#1 study (i.e. 4\%) was considered unusual and attributed to the possible presence of an anomaly on at least one of the cadaveric specimens, as well as to the reduced sample size. For the same Protection condition and Position level, the $\% \Delta$ PRF obtained from Aim \#2 study was much higher (i.e., 36\%), which could explain our initial speculations that the result obtained from Aim \#1 study might have been affected more by specimens' condition and, possibly in a lesser extent, by the sample size. Furthermore, the comparisons of $\% \Delta$ PRF values between Aim \#1 and Aim \#2 studies for gloves G1 and G2 at each Position showed that values from surrogate hand tests were either similar to Aim \#1, for G1 at Metacarpals and G2 at PIP, or higher than \% $\mathrm{PRF}$ from cadaveric hand tests (Table 21). Also, The IPI values calculated using Gel \#4.3 surrogate hand data for all gloves were larger than IPI values from Aim \#1 study, and generally smaller than values reported by Sosa et al. (2019) using both semi-flexible and semi-rigid surrogate hands (Table 22).

The abovementioned differences observed in $\% \Delta$ PRF and IPI values could be attributed to the variability in hand stiffness, strength, and shape between the cadaveric hands and surrogate hand models (i.e., Gel \#4.3, semi-flexible, and semi-rigid surrogate hands). While soft tissues in the Gel \#4.3 surrogate hand developed in this research were represented using Gelatin \#4, soft tissues in the semi-flexible surrogate hand reported in Sosa et al. (2019) were represented using Gelatin \#0, which has the highest hardness value compared to the other gel grades (Table 11). In fact, Gelatin \#0 was tested during the development stages of the present study and resulted in a 
COR value that was $28 \%$ and $23 \%$ larger than COR of cadaveric hands and Gel \#4.3 surrogate hands, respectively (Figure 35). For the semi-rigid surrogate hand, unlike real human hand, the material proprieties and thickness of the hand (wooden rods) across all tested Positions were uniform creating unrealistic and much stiffer surrogate hand.

For cadaveric hand specimens, and based on previous anthropometric studies which reported large variation in human hand anthropometry (Buchholz et al., 1992), it was expected that the stiffness of the tested cadaveric hand specimens obtained from different donors might have some differences. Furthermore, the stiffness and strength of cadaveric hand specimens could be affected by age and previous health conditions. A previous study (Lucas et al., 2008) showed that BMD measured in the forearm of men reduces with age, indicating that cadaveric specimens from different age groups could have different BMD and thus different bone strength. Our Aim \#1 study evaluated bone strength using the cortical index (CI) measurements, and the resulted CI's for all cadaveric specimens ranged from 0.45 to 0.65 indicating the presence of some variability in bone strength (Table 8).

The development of Gel \#4.3 surrogate hand was based on the mean impact response data of all cadaveric specimens, and all the manufactured surrogate hands featured very similar dimensions according to reported $50^{\text {th }}$ percentile measurements. Such feature is expected to reduce variability of tested samples which in turn could reduce the variability in the PRF results. Furthermore, the base material for $3 \mathrm{D}$ printing the bone structure was selected to have close mechanical proprieties to human cortical bone. Therefore, the evolution of surrogate hand denominated Gel \#4.3 was able to capture the average strength and stiffness of real human hand and to minimize the effects of sample condition, variability, and age, often encountered during cadaveric studies. 
Another possible source for the differences observed in $\% \Delta$ PRF and IPI values could be the combined stiffness (interaction effect) of the type of hands used in the test, as well as the materials of the layers that constitute each one of selected gloves. When gloves were impacted without hands, the difference in PRF between the three gloves within both test conditions, full glove and dorsal side tests, ranged from $2 \%$ to $7 \%$ (Figure 38). On the other hand, the difference in PRF between the three gloves when tested using Gel \#4.3 surrogate hands ranged from 5\% to $16 \%$. Such differences could be attributed to the combined stiffness of surrogate hand and materials of gloves.

A reduction in transferred impact force could hypothetically result in injury risk reduction, which was assessed by the ratio between the number of fractures and the total number of impacts for all the tested conditions. Prior to using this measure, it is important to establish the level of similarity in mechanical proprieties between 3D printed bone structure and actual human hand bones. However, biomechanical proprieties testing methods and corresponding equipment were not available for the present study. Instead, an alternative approach was implemented by comparing proportions of injurious impacts for the "no-glove" conditions of the Gel \#4.3 surrogate hands and the cadaveric hands tested with no-glove. Considering all Positions, proportions of injurious impacts for the "no-glove" conditions were $77 \%$ for surrogate hands and $71 \%$ for cadaveric hands (Figure 41 (a) and (b)), which showed less than $8 \%$ difference. Also, comparing proportions from each Position showed similar patterns and comparable values. This level of similarity in mechanical behavior between 3D printed bone structures and cadaveric hands bones was considered acceptable for the purposes of the current study. Thus, proportions of injurious impacts from Gel \#4.3 surrogate hands were used as a protection measure for metacarpal gloves. However, future more detailed studies would require an extensive evaluation 
for the mechanical proprieties of the 3D printed bone structure to assess the level of equivalency to the actual human hand bone structure.

When comparing $\% \Delta$ PRF (Figure 39) with the injurious impact ratio (Figure 42) for the same glove, results for gloves G1 and G2 showed consistent relationships in which more reduction in $\% \Delta \mathrm{PRF}$ were associated with less values of injurious impact proportion and vice versa. This result was expected as the reduction in impact force is hypothetically associated with a reduction in injury risk. On the other hand, while $\% \Delta \mathrm{PRF}$ for glove G3 was $24 \%$, which was close to $\% \Delta \mathrm{PRF}$ for glove $\mathrm{G} 2$ (i.e. $27 \%$ ), the injurious impact ratio for glove $\mathrm{G} 3$ was much higher than the ratio for glove G2 and similar to the ratio for glove G1. Such behavior can be attributed to the differences in thickness and material types used to construct the tested gloves (Table 17 and Table 18). While glove G2 includes goatskin layer and a thick TPR reinforcement which create stronger and stiffer barrier separating the impactor from hands, glove G3 does not include TPR reinforcement and only includes pigskin, foam, and fabric layers. Although these layers of material in glove G3 resulted in dissipating relatively good portion of the transmitted force, their stiffness and thickness may not have been adequate to provide a strong barrier to separate the impactor from the hands. Thus, it failed in providing the same amount of protection against fractures as glove G2. The same argument could also hold true for glove G1 which although includes TPR reinforcement on PIP Position (thinner than TPR of G2), it did not provide as good protection against fracture as glove G2 at the PIP Position and the other Positions.

Comparing gloves G1 and G3, they both had the same injurious impact ratio, yet they had different levels of $\% \Delta \mathrm{PRF}$, which was attributed to following factors: (a) type and thickness of materials on the dorsal side: the average thickness at the region \#1 (Table 18 and Figure 36) for 
gloves G1 and G3 are $4.8 \mathrm{~mm}$ and $5.0 \mathrm{~mm}$, respectively. Thicker and foamier materials could be hypothetically linked to more force dissipation, which was the case in this comparison. Also, the difference in force dissipation ( $\% \Delta \mathrm{PRF})$ could be attributed to the material type of foam padding used in each glove construction. Previous studies reported that the chemical and physical properties of the foam padding have been shown to affect impact force and energy (Chadli et al., 2018; Duncan et al., 2016). While the foam padding on glove G1 is composed of $96 \%$ Polyester and 4\% Spandex, specifications of glove G3 does not include details regarding the type of foam padding. Overall, the separation thickness between the hand and the impactor, and the type/proprieties of material used (e.g. TPR vs. foam padding) could be major factors in preventing fractures; (b) thickness of layers on the palmar side: the impact tests performed on gloves without hand (described in Section 4.3.2) showed some variability between the gloves in terms of the difference in PRF values between full glove and dorsal side tests (Figure 37). Such differences could be attributed to material type and thickness at the anterior side (Table 17 and Table 18; Regions 3 and 4), which was cut off the glove for the performance of the dorsal side impact tests. Therefore, it is speculated that the higher force dissipation observed for glove G3 relative to glove G1 (Figure 39) could be partially attributed to its thicker material layers in the palmar side.

Considering the abovementioned results and discussions, the combination of force dissipation measures (i.e. $\% \Delta \mathrm{PRF}$ and IPI) and injurious impact ratio could provide a more accurate measure of the impact resistance of metacarpal gloves. In case of glove G3, which despite showing a relatively good force dissipation, did not provide relatively equivalent protection from fractures. Such findings further illustrate the added value of using cadaveric or surrogate hands when performing glove impact protection evaluation. In this regard, our impact 
tests on the dorsal side of gloves without hands, which is a technique adopted from the ANSI/ISEA 138-2019 standard, showed that the mean transmitted PRF for the three tested gloves were similar with differences in the range of $2 \%$ to $4 \%$ (Figure 38 ). Such result could indicate that the three gloves are expected to deliver similar levels of protection which was not the case according to the tests performed using cadaveric hands and Gel \#4.3 surrogate hands. This shows that relaying merely on transmitted PRF values from testing gloves without hand could yield unreliable outcomes.

Metacarpal gloves that hypothetically expected to offer higher levels of protection are often bulkier and thicker. Previous studies reported that the use of gloves was linked to reduced dexterity and higher levels of muscle activation (Dianat et al., 2012). A previous study (Fonner, 2019) evaluated the performance levels of the same gloves tested in the present study and reported that glove G3 was associated with significantly lower levels of grip strength relative to gloves G1 and G2. The same study also reported non-significant differences between the three gloves in terms of pinch strength. Another study also investigated the effects of the same gloves tested in the present study on productivity and found no significant differences between their Fitts' throughput values (Sah, 2019). Fitts' throughput is often used as an indicator to measure speed and accuracy of task performance. The same study also reported that the use of glove G2 resulted into slightly higher, yet not significant, muscle activation compared to gloves G1 and G3. Considering these findings that showed roughly similar levels in terms of productivity and performance for the tested gloves, as well as the different levels of impact protection performance shown in the current study, could indicate that it is feasible to design and construct metacarpal gloves with relatively high impact protection without significantly compromising the user's productivity and performance levels. 


\section{Chapter 5. Conclusion}

The prevalence and severity of wrist, hand, and finger injuries in the mining industry were evaluated during our preliminary study presented in Chapter 2. The wrist, hand, and finger injuries accounted for nearly a third of the overall injuries. Over the last two decades, $84 \%$ of the total LWD associated to wrist, hand, and finger injuries was caused by $18 \%$ of the total reported wrist, hand, and finger injuries with a median LWD greater than 30 days. For the severe injuries, the struck by accidents, fractures, and amputation injuries were prevalent and were linked to inadequate hand protection against impact loads.

The use of metacarpal gloves is usually suggested to prevent and reduce hand injuries related to impact accidents. In this study, new testing methods for glove impact protection evaluation were investigated. A series of controlled impact tests were conducted on unprotected and

protected human cadaveric hand specimens (Aim \#1, described in Chapter 3) and surrogate hand specimens (Aim \#2, described in Chapter 4) to quantify the levels of protection provided by different metacarpal gloves. Aim \#1 study involved testing two types of metacarpal gloves by comparing their PRF values and ratio of fractures for specimens tested with and without gloves. Overall, the use of glove on cadaveric hands contributed to a significant reduction in PRF and nearly $44 \%$ reduction in fractures ratio. Aim \#2 study was carried out to minimize the need for testing with cadaveric hands, which often involve logistical difficulties and several limitations. Impact response data from cadaveric hand tests were utilized to develop, calibrate and implement a synthetic surrogate hand with biomechanical properties similar to human hands.

The developed surrogate hand was comprised of a 3D printed bone structure and casted medical-grade synthetic gel to represent soft tissues. PRF and COR values from cadaveric hands were used as reference measurements to adjust and ensure similarity between the global stiffness 
and strength of surrogate hand and the cadaveric hands. The last generation of surrogate hands (Gel \#4.3) showed similar stiffness and strength to cadaveric hands and therefore was used to conduct additional glove impact testing. The same two types of gloves tested with cadaveric hands as well as one more brand were tested using surrogate hands. Depending on the type of glove used and the impacted Position, similar or higher levels of reduction in PRF were observed when using surrogate hands relative to cadaveric hands. The differences were attributed to the variability in condition and age and the small sample size of cadaveric hands. On the other hand, stiffness, strength, and dimensions of all tested surrogate hands were consistent as a result of a systematic manufacturing procedure implemented in this research. Overall, injurious impact (fractures) proportions for gloves tested with surrogate hands and cadaveric hands showed comparable values and trends. Such findings illustrate the suitability of the surrogate hand developed in this study for the evaluation of metacarpal gloves impact-resistance.

\subsection{Industrial applications}

Results of the preliminary study could be used by safety professionals to prioritize problem areas that need immediate actions. The wide range of metacarpals gloves with different designs and features could complicate the glove selection task. Findings of this study showed that different gloves did not perform uniformly under impact loads, indicating the importance of selecting the proper glove for the needed protection. The comparison matrices for the commonly used metacarpal gloves generated from testing cadaveric hand (Aim \#1) and surrogate hand (Aim \#2) specimens under blunt impact loads could aid safety managers for better identification and selection of suitable gloves for different tasks. Furthermore, the methodology and protocol for manufacturing the surrogate hand detailed in this research could be useful in future gloves performance evaluation studies. 
Additionally, results of this study could be utilized to improve current standards for metacarpal gloves classification. Our results illustrate the importance of using a hand (real or surrogate) during glove impact testing which is necessary for calculating the force dissipation measures (i.e. $\% \Delta \mathrm{PRF}$ and IPI) and the injurious impacts proportions. The developed surrogate hand could serve as an accessible and affordable tool to accurately quantify amount of protection provided by gloves. Also, knowledge presented in the current study could be informative for metacarpal glove manufacturers to improve current models of gloves or design new models with more distributed protection. Over the long run, results of this study are anticipated to improve hand safety in the mining industry and other industries with similar risk factors.

\subsection{Study limitations and future work}

Findings of this study are function of the cadaveric hand specimens and experimental design used and therefore subjected to several limitations. First, reduced sample size, previous health conditions, and older age donors are issues encountered in almost all cadaveric based studies, as well as in the current study. Nevertheless, age range of samples tested in the current study better represented the targeted population compared to previous similar studies. The current study tested 13 specimens with a mean age of 53 years (age range 38 to 66), Loshek (2015) tested only six specimens with a mean age of 87 years (age range 76 to 98), and Carpanen et al. (2019) tested 21 specimens with a mean age of 57 years (age range 41 to 73 ). Another limitation is the fixed impact energy level used in this study (10 J nominal, $\sim 7$ to $8 \mathrm{~J}$ measured) which might restrict generalization of results to other levels encountered in various work environments. Future studies could investigate other levels of impact energy and the capability of different gloves at different impact energy levels. Also, impact tests in the current study were designed and curried out to ensure perpendicular impact on the targeted zone of protection (e.g. TPR). 
Impact hazards in real occupational settings may target the hand at different angles and depending on the glove design, some of the TPR protection or padding may be completely inadequate in preventing injuries. Finally, the surrogate hand was designed to represent a $50^{\text {th }}$ percentile male hand for simplification purposes. Previous anthropometric studies showed large variation in human hand dimensions, which could limit the validity of current results under different circumstances. Future studies should investigate surrogate hands with other dimensions as well as postures other than the semi-flat configuration adopted in this research. 


\section{References}

Abraham, M. K., \& Scott, S. (2010). The Emergent Evaluation and Treatment of Hand and Wrist Injuries. Emergency Medicine Clinics of North America, 28(4), 789-809. https://doi.org/10.1016/j.emc.2010.06.004

Ackland, D., Robinson, D., Lee, P. V. S., \& Dimitroulis, G. (2018). Design and clinical outcome of a novel 3D-printed prosthetic joint replacement for the human temporomandibular joint. Clinical Biomechanics, 56(July 2017), 52-60. https://doi.org/10.1016/j.clinbiomech.2018.05.006

Alessa, F. M., Nimbarte, A. D., \& Sosa, E. M. (2020). Incidences and severity of wrist, hand, and finger injuries in the u.s. mining industry. Safety Science, 29, 104792.

Aryaei, A., Hashemnia, K., \& Jafarpur, K. (2010). Experimental and numerical study of ball size effect on restitution coefficient in low velocity impacts. International Journal of Impact Engineering, 37(10), 1037-1044. https://doi.org/10.1016/j.ijimpeng.2010.04.005

Ashok Kumar, D., Anburajan, M., \& Snekhalatha, U. (2018). Evaluation of low bone mass and prediction of fracture risk using metacarpal radiogrammetry method: a comparative study with DXA and X-ray phantom. International Journal of Rheumatic Diseases, 21(7), 1350 1371. https://doi.org/10.1111/1756-185X.13326

Barnett, E., \& Nordin, B. E. C. (1960). The radiological diagnosis of osteoporosis: a new approach. Clinical Radiology, 11(3), 166-174.

Bewick, V., Cheek, L., \& Ball, J. (2003). Statistics review 8: Qualitative data - Tests of association. Critical Care, 8(1), 46-53. https://doi.org/10.1186/cc2428

Bonin, S. J., Luck, J. F., Bass, C. R., Gardiner, J. C., Onar-Thomas, A., Asfour, S. S., \& Siegmund, G. P. (2017). Dynamic Response and Residual Helmet Liner Crush Using Cadaver Heads and Standard Headforms. Annals of Biomedical Engineering, 45(3), 656667. https://doi.org/10.1007/s10439-016-1712-5

Boonen, S., Vanderschueren, D., Callewaert, F., \& Haentjens, P. (2009). Aging and Bone Loss. In Osteoporosis in men: the effects of gender on skeletal health (2nd ed.). Elsevier.

Breuer, J., Höffer, E.-M., \& Hummitzsch, W. (2002). Rate of occupational accidents in the mining industry since 1950 - a successful approach to prevention policy. Journal of Safety Research, 33(1), 129-141. https://www.sciencedirect.com/science/article/pii/S0022437502000099

Buchholz, B., Armstrong, T. J., \& Goldstein, S. A. (1992). Anthropometric data for describing the kinematics of the human hand. Ergonomics, 35(3), 261-273.

Bureau of Labor Statistics. (2017a). TABLE R2. Number of nonfatal occupational injuries and illnesses involving days away from work by industry and selected parts of body affected by injury or illness, private industry. https://www.bls.gov/iif/oshwc/osh/case/cd_r2_2017.htm

Bureau of Labor Statistics. (2017b). TABLE R65. Number of nonfatal occupational injuries and illnesses involving days away from work by days away from work groups and median 
number of days away from work by industry, private industry.

https://www.bls.gov/iif/oshwc/osh/case/cd_r65_2017.htm

Byrnes, K., Abramczyk, J., Berliner, J., Irwin, A., Jensen, J., Kowsika, M., Mertz, H. J., Rouhana, S. W., Scherer, R., Shi, Y., Sutterfield, A., Xu, L., \& Beach, P. V. (2002). ES-2 Dummy Biomechanical Responses. 46th Stapp Car Crash Conference, 46(November).

Canny, J. (1986). A Computational Approach to Edge Detection. IEEE Transactions on Pattern Analysis and Machine Intelligence, PAMI-8(6), 679-698. https://doi.org/10.1109/TPAMI.1986.4767851

Cantrell, J., Rohde, S., Damiani, D., Gurnani, R., Di Sandro, L., Anton, J., Young, A., Jerez, A., Steinbach, D., Kroese, C., \& Ifju, P. (2017). Experimental characterization of the mechanical properties of 3D printed ABS and polycarbonate parts. Conference Proceedings of the Society for Experimental Mechanics Series, 3, 89-105. https://doi.org/10.1007/978-3319-41600-7_11

Carpanen, D., Kedgley, A. E., Shah, D. S., Edwards, D. S., Plant, D. J., \& Masouros, S. D. (2019). Injury risk of interphalangeal and metacarpophalangeal joints under impact loading. Journal of the Mechanical Behavior of Biomedical Materials, 97(September 2018), 306311. https://doi.org/10.1016/j.jmbbm.2019.05.037

Chadli, S., Ababou, N., Ababou, A., \& Ouadahi, N. (2018). Quantification of boxing gloves damping: Method and apparatus. Measurement, 129, 504-517.

Chung, K. C., Spilson, S. V, \& Arbor, A. (2001). The Frequency and Epidemiology of Hand and Forearm Fractures in the United States. The Journal of Hand Surgery, 26(5), 908-915. https://doi.org/10.1053/jhsu.2001.26322

Clavero, J. A., Golanó, P., Farinas, O., Alomar, X., Monill, J. M., \& Esplugas, M. (2003). Extensor mechanism of the fingers: MR imaging-anatomic correlation. Radiographics, 23(3), 593-611.

Coleman, P. J., \& Kerkering, J. C. (2007). Measuring mining safety with injury statistics: Lost workdays as indicators of risk. Journal of Safety Research, 38(5), 523-533. https://www.sciencedirect.com/science/article/pii/S0022437507001004

Davasaksan, A., Durusoy, R., Bal, E., Kayalar, M., Ada, S., \& Tanik, F. A. (2012). Risk factors for occupational hand injuries: Relationship between agency and finger. American Journal of Industrial Medicine, 55(5), 465-473. https://doi.org/10.1002/ajim.22016

De Putter, C. E., Van Beeck, E. F., Polinder, S., Panneman, M. J. M., Burdorf, A., Hovius, S. E. R., \& Selles, R. W. (2016). Healthcare costs and productivity costs of hand and wrist injuries by external cause: A population-based study in working-age adults in the period 2008-2012. Injury, 47(7), 1478-1482. https://doi.org/10.1016/j.injury.2016.04.041

Dianat, I., Haslegrave, C. M., \& Stedmon, A. W. (2012). Methodology for evaluating gloves in relation to the effects on hand performance capabilities: A literature review. Ergonomics, 55(11), 1429-1451. https://doi.org/10.1080/00140139.2012.708058

Dolez, P., Soulati, K., Gauvin, C., Lara, J., \& Vu-Khanh, T. (2010). Studies and Research Projects, Technical Guide RG-738, Information Document for Selecting Gloves for 
Protection against Mechanical Hazards. Institut de recherché Robert-Sauvé en santé et en sécurité du travail (IRSST).

Duncan, O., Foster, L., Senior, T., Allen, T., \& Alderson, A. (2016). A Comparison of Novel and Conventional Fabrication Methods for Auxetic Foams for Sports Safety Applications. Procedia Engineering, 147(0), 384-389. https://doi.org/10.1016/j.proeng.2016.06.323

Eisele, A., Dereskewitz, C., Kus, S., Oberhauser, C., Rudolf, K., Coenen, M., Sauerbier, M., Bickert, B., Struckmann, V., Kneser, U., Daigeler, A., \& Höffken, O. (2018). Factors affecting time off work in patients with traumatic hand injuries - A bio-psycho-social perspective. Injury, 49(10), 1822-1829. https://doi.org/10.1016/j.injury.2018.07.012

Fernandez-Vicente, M., Calle, W., Ferrandiz, S., \& Conejero, A. (2016). Effect of Infill Parameters on Tensile Mechanical Behavior in Desktop 3D Printing. 3D Printing and Additive Manufacturing, 3(3), 183-192. https://doi.org/10.1089/3dp.2015.0036

Fonner, A. A. (2019). Effect of Metacarpal Gloves on Pinch and Grip Strength.

Fox, K. M., Kimura, S., Powell-Threets, K., \& Plato, C. C. (1995). Radial and Ulnar Cortical Thickness of the Second Metacarpal. Journal of Bone and Mineral Research, 10(12), 1930 1934.

Garrett, J. W. (1971). The Adult Human Hand : Some Anthropometric and Biomechanical Considerations. Human Factors, 13(2), 117-131.

Glencross, B., \& Agarwal, S. C. (2011). An investigation of cortical bone loss and fracture patterns in the neolithic community of Çatalhöyük, Turkey using metacarpal radiogrammetry. Journal of Archaeological Science, 38(3), 513-521. https://doi.org/10.1016/j.jas.2010.10.004

Gosling, J. A., Harris, P. F., Humpherson, J. R., Whitmore, I., \& Willan, P. L. (2016). Human Anatomy, Color Atlas and Textbook. Elsevier Health Sciences.

Grayson, R. L., Althouse, R. C., Winn, G. L., \& Klishis, M. J. (1998). A New Injury Analysis Methodology for Developing Prioritized Workplace Intervention Strategies. Applied Occupational and Environmental Hygiene, 13(1), 41-52.

Greenwald, R. M., Janes, P. C., Swanson, S. C., \& Mcdonald, T. R. (2010). The American Journal of Sports Medicine Dynamic Impact Response of Human Cadaveric Forearms Using a Wrist Brace. 26(6), 2-7.

Greiner, T. M. (1991). Hand Anthropometry of U.S. Army Personell. In ARMY NATICK RESEARCH DEVELOPMENT AND ENGINEERING CENTER: Vol. TR-92/011. http://oai.dtic.mil/oai/oai?verb=getRecord \&amp; metadataPrefix=html\&amp;identifier=AD A244533

Groves, W. A., Kecojevic, V. J., \& Komljenovic, D. (2007). Analysis of fatalities and injuries involving mining equipment. Journal of Safety Research, 38(4), 461-470. https://doi.org/10.1016/j.jsr.2007.03.011

Hardy, W. N., Mason, M. J., Foster, C. D., Shah, C. S., Kopacz, J. M., Yang, K. H., King, A. I., Bishop, J., Bey, M., Anderst, W., \& Tashman, S. (2007). A study of the response of the 
human cadaver head to impact. Stapp Car Crash Journal, 51, 17-80.

Haron, A., \& Ismail, K. A. (2012). Coefficient of restitution of sports balls: A normal drop test. IOP Conference Series: Materials Science and Engineering, 36(1). https://doi.org/10.1088/1757-899X/36/1/012038

Harrison, C. R., \& Robinette, K. M. (2002). Caesar : Summary Statistics for the Adult. In Interface (Issue December 1997).

Headman, Z. C., Matson, M. C., Schneider, R. P., Potter, J. L., Loguda-Summers, D. L., Bhatia, S., \& Kondrashova, T. (2020). Developing Neuraxial and Regional Pain Procedural Skills Through Innovative 3-Dimensional Printing Technology. The Journal of the American Osteopathic Association, 120(4), 273. https://doi.org/10.7556/jaoa.2020.044

Hill, C., Riaz, M., Mozzam, A., \& Brennen, M. D. (1998). A Regional Audit of Hand and Wrist Injuries. Journal of Hand Surgery, 23(2), 196-200. https://doi.org/10.1016/S02667681(98)80174-5

Humimic Medical, Fort Smith, AR. (n.d.). Retrieved June 10, 2020, from https://humimic.com/product-category/medical-gels/medical-gels-medical-gels/

Hummel, A., Barker, R., Lyons, K., Deaton, A. S., \& Morton-Aslanis, J. (2011). Development of Instrumented Manikin Hands for Characterizing the Thermal Protective Performance of Gloves in Flash Fire Exposures. Fire Technology, 47(3), 615-629. https://doi.org/10.1007/s10694-010-0190-9

Ives, R., \& Brickley, M. B. (2004). A procedural guide to metacarpal radiogrammetry in archaeology. International Journal of Osteoarchaeology, 14(1), 7-17. https://doi.org/10.1002/oa.709

Jardini, A. L., Larosa, M. A., Filho, R. M., Zavaglia, C. A. D. C., Bernardes, L. F., Lambert, C. S., Calderoni, D. R., \& Kharmandayan, P. (2014). Cranial reconstruction: 3D biomodel and custom-built implant created using additive manufacturing. Journal of Cranio-Maxillofacial Surgery, 42(8), 1877-1884. https://doi.org/10.1016/j.jcms.2014.07.006

Javadi, M., Saeedi, G., \& Shahriar, K. (2017). Developing a New Probabilistic Approach for Risk Analysis, Application in Underground Coal Mining. Journal of Failure Analysis and Prevention, 17(5), 989-1010. https://doi.org/10.1007/s11668-017-0325-0

Jian, S., Lian-guo, W., Hua-lei, Z., \& Yi-feng, S. (2009). Application of fuzzy neural network in predicting the risk of rock burst. Procedia Earth and Planetary Science, 1(1), 536-543. https://doi.org/10.1016/j.proeps.2009.09.085

Jin, K., Lombardi, D. A., Courtney, T. K., Sorock, G. S., Li, M., Pan, R., Wang, X., Lin, J., Liang, Y., \& Perry, M. J. (2010). Patterns of work-related traumatic hand injury among hospitalised workers in the People's Republic of China. Injury Prevention, 16(1), 42-49. https://doi.org/10.1136/ip.2008.019737

Khalid, N. E. A., Manaf, M., \& Aziz, M. E. (2010). An empirical study using line profile histogram approximation of edge detection algorithms. 2010 Second International Conference on Computer Engineering and Applications, 1, 344-348. https://doi.org/10.1109/ICCEA.2010.74 
Kovacs, L., Grob, M., Zimmermann, A., Eder, M., Herschbach, P., Henrich, G., Zimmer, R., Biemer, E., \& Papadopulos, N. A. (2011). Quality of life after severe hand injury. Journal of Plastic, Reconstructive \& Aesthetic Surgery, 64(11), 1495-1502. https://doi.org/10.1016/j.bjps.2011.05.022

Laflamme, L., \& Blank, V. L. C. (1996). Age-related Accident Risks: Longitudinal Study of Swedish Iron Ore Miners. American Journal of Industrial Medicine, 30(4), 479-487. https://onlinelibrary.wiley.com/doi/abs/10.1002/(SICI)10970274(199610)30:4\%3C479::AID-AJIM14\%3E3.0.CO;2-1

Lanzotti, A., Grasso, M., Staiano, G., \& Martorelli, M. (2015). The impact of process parameters on mechanical properties of parts fabricated in PLA with an open-source 3-D printer. Rapid Prototyping Journal, 21(5), 604-617. https://doi.org/10.1108/RPJ-09-2014-0135

Larsen, C. F., Mulder, S., Mette, A., Johansen, T., \& Stam, C. (2004). The epidemiology of hand injuries in the Netherlands and Denmark. European Journal of Epidemiology, 19(4), 323327. https://link.springer.com/article/10.1023/B:EJEP.0000024662.32024.e3

Lee, T., Anderson, C., \& Kraus, J. (1993). Acute traumatic injuries in underground bituminous coal miners. American Journal of Industrial Medicine, 23(3), 407-415.

Lim, K. H. A., Loo, Z. Y., Goldie, S. J., Adams, J. W., \& McMenamin, P. G. (2016). Use of 3D printed models in medical education: A randomized control trial comparing 3D prints versus cadaveric materials for learning external cardiac anatomy. Anatomical Sciences Education, 9(3), 213-221. https://doi.org/10.1002/ase.1573

Lind, S. (2008). Types and sources of fatal and severe non-fatal accidents in industrial maintenance. International Journal of Industrial Ergonomics, 38(11-12), 927-933. https://doi.org/10.1016/j.ergon.2008.03.002

Liu, K., Greencorn, D. J., Aponte, D. I., Robbins, S. M., \& Pearsall, D. J. (2019). Comparison of surrogate 50th percentile human headforms to an adult male sample using threedimensional modeling and principal component analysis. Proceedings of the Institution of Mechanical Engineers, Part P: Journal of Sports Engineering and Technology, 233(3), 432-442. https://doi.org/10.1177/1754337119840848

Loshek, P. D. (2015). “Classification of Adequate Impact Protection for Hands." In Theses and Dissertations. http://dc.uwm.edu/etd/974/

Louden, A. E. (2019). WorldSID 50th Percentile Male Dummy Seating Procedure Evaluation and Revision (Issue April).

Lowery, J. T., Glazner, J., Borgerding, J. A., Bondy, J., Lezotte, D. C., \& Kreiss, K. (2000). Analysis of construction injury burden by type of work. American Journal of Industrial Medicine, 37(4), 390-399. https://doi.org/10.1002/(SICI)10970274(200004)37:4<390::AID-AJIM9>3.0.CO;2-0

Lucas, R., Silva, C., Costa, L., Araújo, D., \& Barros, H. (2008). Male ageing and bone mineral density in a sample of Portuguese men. Acta Reumatologica Portuguesa, 33(3).

MacDonald, P. L., \& Gardner, R. C. (2000). Type I error rate comparisons of post hoc procedures for i j chi-square table. Educational and Psychological Measurement, 60(5), 
$735-754$.

Margolis, K. A. (2010). Underground coal mining injury: A look at how age and experience relate to days lost from work following an injury. Safety Science, 48(4), 417-421. https://doi.org/10.1016/j.ssci.2009.12.015

MATLAB Text Analytics Toolbox User's Guide R2019b (No. R2019b). (2019). The MathWorks, Inc.

Mital, A., Pennathur, A., \& Kansal, A. (2000). Nonfatal occupational injuries in the United States Part III-injuries to the upper extremities. International Journal of Industrial Ergonomics, 25(2), 151-169.

Mix, A. W., \& Giacomin, A. J. (2011). Standardized Polymer Durometry. Journal of Testing and Evaluation, 39(4), 103205. https://doi.org/10.1520/jte103205

Montgomery, D. C. (2012). Design and analysis of experiments. John wiley \& sons.

Moore, S. M., Porter, W. L., \& Dempsey, P. G. (2009). Fall from equipment injuries in U.S. mining: Identification of specific research areas for future investigation. Journal of Safety Research, 40(6), 455-460. https://doi.org/10.1016/j.jsr.2009.10.002

Morgan, W. J., \& Harrop, S. N. (1985). Hand injuries in south Wales coal miners. British Journal of Industrial Medicine, 42(12), 844-847.

Nasarwanji, M. F., Pollard, J., \& Porter, W. (2018). An analysis of injuries to front-end loader operators during ingress and egress. International Journal of Industrial Ergonomics, 65, 84-92. https://doi.org/10.1016/j.ergon.2017.07.006

Nowrouzi-Kia, B., Sharma, B., Dignard, C., Kerekes, Z., Dumond, J., Li, A., \& Larivière, M. (2017). Systematic review: Lost-time injuries in the US mining industry. Occupational Medicine, 67(6), 442-447. https://doi.org/10.1093/occmed/kqx077

Nowrouzi-Kia, Behdin, Gohar, B., Casole, J., Chidu, C., Dumond, J., McDougall, A., \& Nowrouzi-Kia, B. (2018). A systematic review of lost-time injuries in the global mining industry. Work, 60(1), 49-61. https://doi.org/10.3233/WOR-182715

Öchsner, A., Ahmed, W., Fish, J., \& Belytschko, T. (2011). Biomechanics of hard tissues. Weinheim: Wiley.

OpenStax, C. N. X. (2017). Anatomy and physiology. In Management of Adult Glioma in Nursing Practice. https://openstax.org/details/books/anatomy-and-physiology

Pang, G., Futter, C., Pincus, J., Dhanani, J., \& Laupland, K. B. (2020). Development and testing of a low cost simulation manikin for extracorporeal cardiopulmonary resuscitation (ECPR) using 3-dimensional printing. Resuscitation, 149(December 2019), 24-29. https://doi.org/10.1016/j.resuscitation.2020.01.032

Paul, P. S. (2009). Predictors of work injury in underground mines - an application of a logistic regression model. Mining Science and Technology (China), 19(3), 282-289. https://doi.org/10.1016/S1674-5264(09)60053-3

Plato, C. C., \& Norris, A. H. (1980). Bone Measurements of the Second Metacarpal and Grip 
Strength. Human Biology, 52(1), 131-149.

Pollard, J., Heberger, J., \& Dempsey, P. G. (2014). Maintenance and repair injuries in US mining. Journal of Quality in Maintenance Engineering, 20(1), 20-31. https://doi.org/10.1108/JQME-02-2013-0008

Poplin, G. S., Miller, H. B., Ranger-moore, J., Bofinger, C. M., Kurzius-Spencer, M., Harris, R. B., \& Burgess, J. L. (2008). International evaluation of injury rates in coal mining : A comparison of risk and compliance-based regulatory approaches. Safety Science, 46(8), 1196-1204. https://doi.org/10.1016/j.ssci.2007.06.025

Putter, C. E. De, Selles, R. W., Polinder, S., \& Beeck, E. F. Van. (2012). Economic impact of hand and wrist injuries: health-care costs and productivity costs in a population-based study. JBJS, 94(9), 1-7.

Raheja, A. (2008). Automated analysis of metacarpal cortical thickness in serial hand radiographs. Wright State University.

Reardon, L. M., Heberger, J. R., \& Dempsey, P. G. (2014). Analysis of Fatalities During Maintenance and Repair Operations in the U.S. Mining Sector. IIE Transactions on Occupational Ergonomics and Human Factors, 2(1), 27-38. https://doi.org/10.1007/s10995-015-1800-4.Alcohol

Reilly, D. T., \& Burstein, A. H. (1974). The Mechanical Properties of Cortical Bone. JBJS, 56(5), 1001-1022.

Risler, Z., Magee, M. A., Mazza, J. M., Goodsell, K., Au, A. K., Lewiss, R. E., Pugliese, R. S., \& Ku, B. (2018). A Three-dimensional Printed Low-cost Anterior Shoulder Dislocation Model for Ultrasound-guided Injection Training. Cureus, 10(11). https://doi.org/10.7759/cureus.3536

Robinson, L. S., Sarkies, M., Brown, T., \& O’Brien, L. (2016). Direct, indirect and intangible costs of acute hand and wrist injuries: A systematic review. Injury, 47(12), 2614-2626. https://doi.org/10.1016/j.injury.2016.09.041

Rui, P., \& Kang, K. (2015). National Hospital Ambulatory Medical Care Survey : 2015 Emergency Department Summary Tables. https://www.cdc.gov/nchs/data/nhamcs/web_tables/2015_ed_web_tables.pdf

Sah, S. (2019). Can Fitts' Throughput Be A Predictor Of Muscle Fatigue - A Study Based On Metacarpal Gloves?

Sammarco, J. J., Podlesny, A., Rubinstein, E. N., \& Demich, B. (2016). An analysis of roof bolter fatalities and injuries in U.S. mining. Transactions of Society for Mining, Metallurgy, and Exploration, Inc, 340(1), 11-20. https://doi.org/10.19150/trans.7322

Sandeep, C. S., Senetakis, K., Cheung, D. K. H., Choi, C. E., Wang, Y., Coop, M., \& Ng, C. W. W. (2020). Experimental study on the coefficient of restitution of grain against block interfaces for natural and engineered materials. Canadian Geotechnical Journal, 1-44. https://doi.org/10.1139/cgj-2018-0712

Santos, B. R., Porter, W. L., \& Mayton, A. G. (2010). An Analysis of Injuries to Haul Truck 
Operators in the U.S. Mining Industry. In Proceedings of the Human Factors and Ergonomics Society Annual Meeting, 54(21), 1870-1874.

Schuurman, A. H., \& Kauer, J. M. G. (2002). Impact load on the triangular fibrocartilage of the wrist: A cadaver study. Journal of Surgical Research, 103(2), 129-133. https://doi.org/10.1006/jsre.2001.6344

Shubhangi, D. C., Raghavendra, S., \& Hiremath, P. S. (2012). Edge Detection of Femur Bones in $\mathrm{X}$-ray images A comparative study of Edge Detectors. International Journal of Computer Applications, 42(2), 13-16. https://doi.org/10.5120/5663-7696

Sorock, G. S., Lombardi, D. A., Hauser, R. B., Eisen, E. A., Herrick, R. F., \& Mittleman, M. A. (2001). A case-crossover study of occupational traumatic hand injury: Methods and initial findings. American Journal of Industrial Medicine, 39(2), 171-179. https://doi.org/10.1002/1097-0274(200102)39:2<171::AID-AJIM1004>3.0.CO;2-0

Sorock, G. S., Lombardi, D. A., Peng, D. K., Hauser, R., Eisen, E. A., Herrick, R. F., \& Mittleman, M. A. (2004). Glove Use and the Relative Risk of Acute Hand Injury: A CaseCrossover Study. Journal of Occupational and Environmental Hygiene, 1(3), 182-190. https://doi.org/10.1080/15459620490424500

Sosa, E. M., Dean, J. M., \& Brady, J. R. (2019). Performance under Impact Loads of Metacarpal Gloves used in the Mining Industry. Annual International Occupational Ergonomics and Safety.

Sun, J., \& Zhang, F. Q. (2012). The Application of Rapid Prototyping in Prosthodontics. Journal of Prosthodontics, 21(8), 641-644. https://doi.org/10.1111/j.1532-849X.2012.00888.x

SynDaver Lab, Tampa, F. (n.d.). No Title. https://syndaver.com/

Trotta, A., Ní Annaidh, A., Burek, R. O., Pelgrims, B., \& Ivens, J. (2018). Evaluation of the head-helmet sliding properties in an impact test. Journal of Biomechanics, 75, 28-34. https://doi.org/10.1016/j.jbiomech.2018.05.003

U.S. Department of Labor Mine Safety and Health Administration [MSHA]. (1986). Report on 30 CFR Part 50. https://www.msha.gov/sites/default/files/Support_Resources/Forms/rptonpart50.pdf

Ural, S., \& Demirkol, S. (2008). Evaluation of occupational safety and health in surface mines. Safety Science, 46(6), 1016-1024. https://doi.org/10.1016/j.ssci.2007.11.010

Weston, E., Nasarwanji, M., \& Pollard, J. (2016). Identification of Work-Related Musculoskeletal Disorders in Mining. Journal of Safety, Health and Environmental Research, 12(1), 274-283. https://doi.org/10.1001/jama.2014.15298.Metformin

White, T. D., \& Folkens, P. A. (2005). The human bone manual. Elsevier.

Wu, J. Z., Pan, C. S., \& Wimer, B. M. (2019). Effects of Impactor Mass in Top Impact Tests in Evaluation of Shock Absorption Performance of Construction Helmets : A Preliminary Study. 97819384965-97819384967.

Wu, Y. Y., Rajaraman, M., Guth, J., Salopek, T., Altman, D., Sangimino, M., \& Shimada, K. 
(2018). A High-fidelity Tactile Hand Simulator as a Training Tool to Develop Competency in Percutaneous Pinning in Residents. JAAOS: Global Research and Reviews, 2(7), e028. https://doi.org/10.5435/jaaosglobal-d-18-00028

Wu, Y. Y., Rajaraman, M., Lee, B., Plakseychuk, A., \& Shimada, K. (2016). A patient-specific flexible 3D printed orthopedic model for training and teaching of clubfoot correction surgery. 3D Printing and Additive Manufacturing, 3(2), 99-105. https://doi.org/10.1089/3dp.2016.0005

Yoganandan, N., Chirvi, S., Pintar, F. A., Uppal, H., Schlick, M., Banerjee, A., Voo, L., Merkle, A., \& Kleinberger, M. (2016). Foot-Ankle Fractures and Injury Probability Curves from Post-mortem Human Surrogate Tests. Annals of Biomedical Engineering, 44(10), 29372947. https://doi.org/10.1007/s10439-016-1598-2

Zygote, American Fork, U. 84003. (2015). Zygote, American Fork, UT 84003. 


\section{Appendix A: Normality assumption test}

\section{Aim \#1}

i. This part includes the normality assumption tests performed for Section 3.2.4.4.

1. Residual normality:

The following figure shows the residual normal quantile plot which indicates that the residuals are normally distribution. Also, the goodness-of-fit test showed resulted in a Pvalue of 0.5233 which further indicates a normally distributed data.

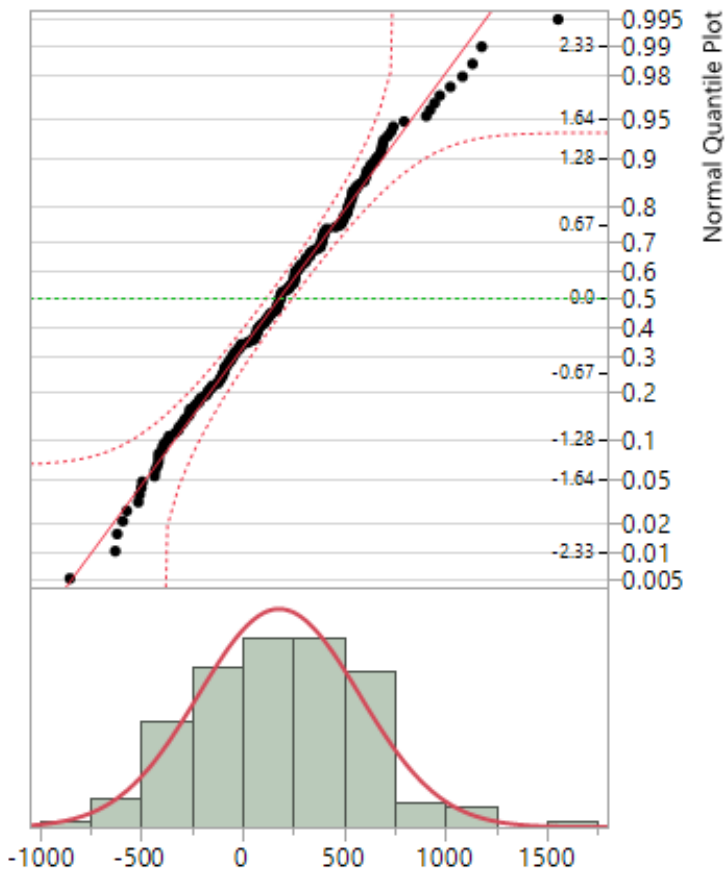

\section{Goodness-of-Fit Test \\ Shapiro-Wilk W Test

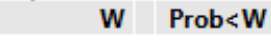 \\ $0.993198 \quad 0.5233$}

Note: $\mathrm{Ho}=$ The data is from the Normal distribution. Small p-values reject Ho. 
2. Constant variance:

The assumption of constant variance was tested by plotting residual versus predicted values as illustrated in the following figure. According to evident from this figure, the points are scattered randomly above and below the center line which indicates a constant variance.

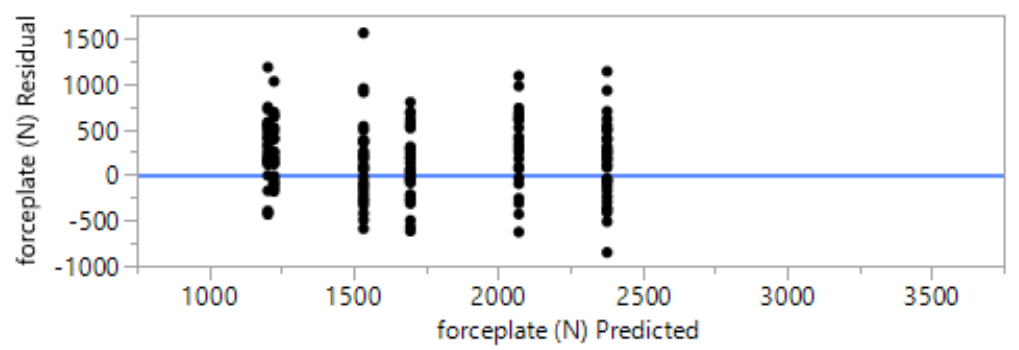

\section{Aim \#2}

ii. This part includes the normality assumption tests performed for Section 4.3.4.3.

1. Residual normality:

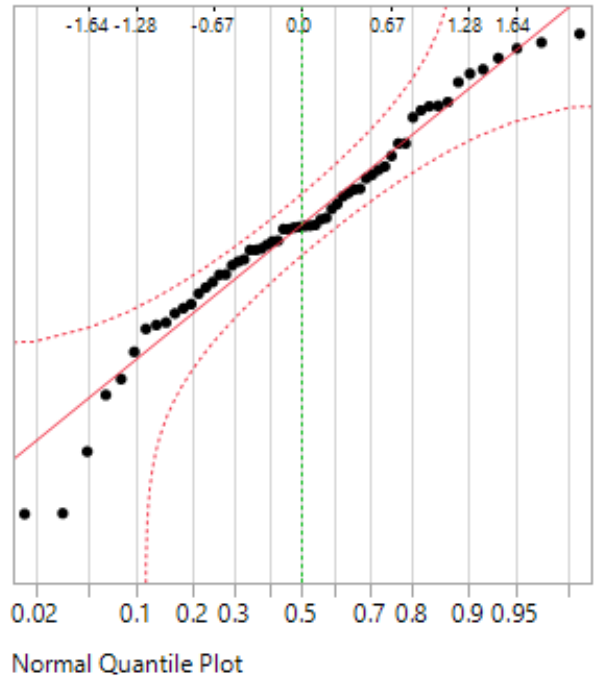




\begin{tabular}{|c|c|}
\hline Goodness & f-Fit Test \\
\hline Shapiro-Wilk & V Test \\
\hline w & Prob $<W$ \\
\hline 0.965378 & 0.0865 \\
\hline $\begin{array}{l}\text { Note: } \mathrm{Ho}= \\
\text { reject } \mathrm{Ho} \text {. }\end{array}$ & data is from the Normal distribution. Small p-values \\
\hline
\end{tabular}

2. Constant variance:

The assumption of constant variance was tested by plotting residual versus predicted values as illustrated in the following figure. According to evident from this figure, the points are scattered randomly above and below the center line which indicates a constant variance.

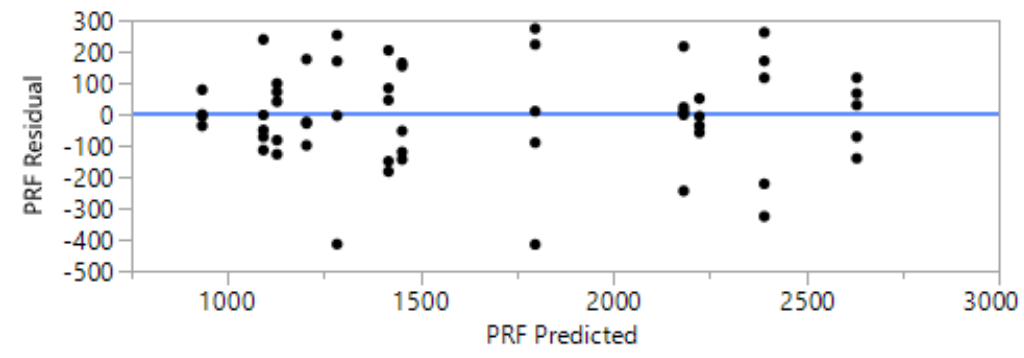




\section{Appendix B: ANOVA tables}

\section{ANOVA tables for Aim \#1}

\begin{tabular}{|l|r|r|r|r|r|}
\hline \multicolumn{2}{l}{ Fixed Effect Tests } & & & & \\
\hline Source & Nparm & DF & DFDen & F Ratio & Prob > F \\
Protection & 1 & 1 & 25.76 & 7.3072 & $0.0120^{*}$ \\
Position & 2 & 2 & 184 & 92.0970 & $<.0001^{\star}$ \\
Protection*Position & 2 & 2 & 184 & 0.8394 & 0.4336
\end{tabular}

The student's t-test tables:
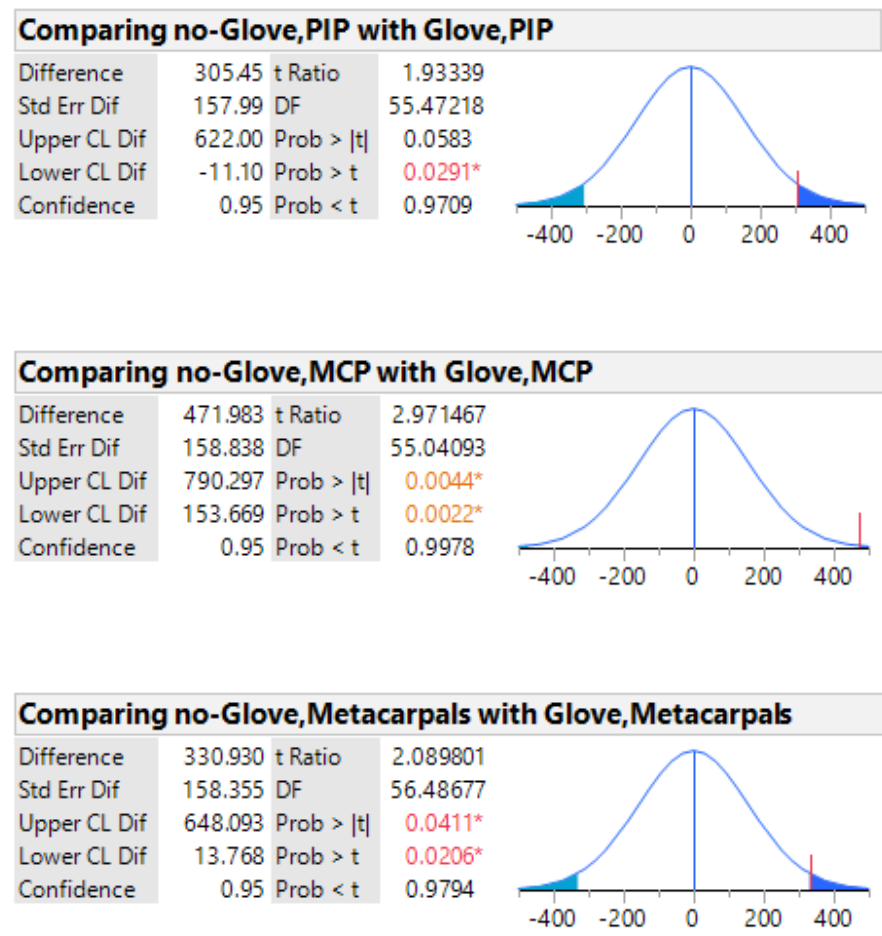


\section{ANOVA tables for Aim \#2}

\begin{tabular}{|c|c|c|c|c|c|}
\hline \multicolumn{6}{|l|}{ Effect Tests } \\
\hline Source & Nparm & DF & $\begin{array}{r}\text { Sum of } \\
\text { Squares }\end{array}$ & F Ratio & Prob $>F$ \\
\hline Condition & 3 & 3 & 2584395 & 30.3962 & $<.0001^{*}$ \\
\hline Pisition & 2 & 2 & 15620849 & 275.5858 & $<.0001^{*}$ \\
\hline Condition*Pisition & 6 & 6 & 153723 & 0.9040 & 0.5000 \\
\hline
\end{tabular}

The student's t-test tables:

- LSMeans Differences Student's t

$\alpha=0.050 t=2.01063$

\begin{tabular}{|l|r|r|r}
\hline Level & & & $\begin{array}{r}\text { Least } \\
\text { Sq Mean }\end{array}$ \\
\hline NG & A & & 1960.4000 \\
G1 & B & 1697.3333 \\
G3 & & C & 1493.0000 \\
G2 & & C & 1428.2667
\end{tabular}

Levels not connected by same letter are significantly different.

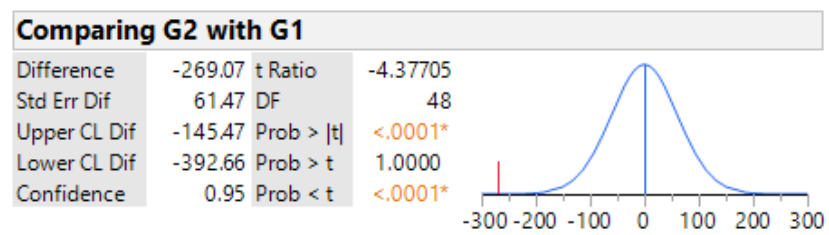

\section{Comparing G3 with G1}

\section{Difference $\quad-204.33$ t Ratio}

Std Err Dif 61.47 DF

Upper CL Dif $\quad-80.74$ Prob $>|t| \quad 0.0017^{\star}$

Lower CL Dif $\quad-327.93$ Prob $>$ t 0.9991

Confidence

0.95 Prob $<\mathrm{t} \quad 0.0009^{*}$

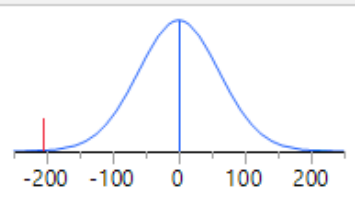

\section{Comparing G3 with G2}

\begin{tabular}{|c|c|c|}
\hline Difference & 64.73 t Ratio & 1.053052 \\
\hline Std Err Dif & $61.47 \mathrm{DF}$ & 48 \\
\hline Upper CL Dif & 188.33 Prob $>|t|$ & 0.2976 \\
\hline Lower CL Dif & -58.86 Prob $>t$ & 0.1488 \\
\hline Confidence & 0.95 Prob $<\mathrm{t}$ & 0.8512 \\
\hline
\end{tabular}

\section{Comparing NG with G1}

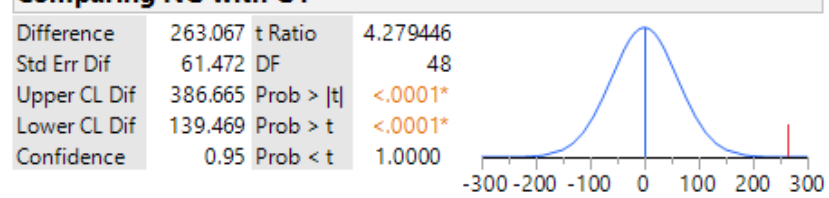


Comparing NG with G2

Difference $\quad 532.133$ t Ratio 8.656497

\begin{tabular}{l|l} 
Std Err Dif & 61.472 DF
\end{tabular}

Upper CL Dif 655.731 Prob $>|t| \quad<.0001^{*}$

Lower CL Dif 408.535 Prob $>t \quad<, 0001^{*}$

Confidence $\quad 0.95$ Prob $<\mathrm{t} \quad 1.0000$

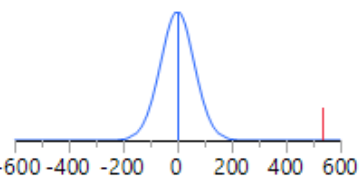

Comparing NG with G3

Difference $\quad 467.400$ t Ratio $\quad 7.603445$

$\begin{array}{lll}\text { Std Err Dif } & 61.472 \text { DF } & 48\end{array}$

Upper CL Dif 590.998 Prob $>|t| \quad<.0001^{\star}$

Lower CL Dif $\quad 343.802$ Prob $>t \quad<.0001^{*}$

Confidence $\quad 0.95$ Prob $<\mathrm{t} \quad 1.0000$

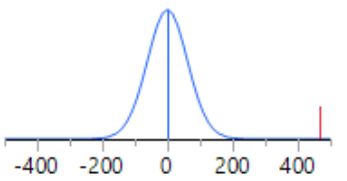

\section{$1-$ LSMeans Differences Student's t}

$\alpha=0.050 \mathrm{t}=2.01063$

\begin{tabular}{l|l|r|r}
\hline Level & & & $\begin{array}{r}\text { Least } \\
\text { Sq Mean }\end{array}$ \\
\hline PIP & A & & 2357.4000 \\
MCP & B & 1386.5000 \\
Metacarpals & & C & 1190.3500
\end{tabular}

Levels not connected by same letter are significantly different.

\section{Comparing Metacarpals with MCP}

Difference $\quad-196.15$ t Ratio $\quad-3.68451$

$\begin{array}{llr}\text { Std Err Dif } & 53.24 \text { DF }\end{array}$

Upper CL Dif $\quad-89.11$ Prob $>|t| \quad 0.0006^{*}$

Lower CL Dif $\quad-303.19$ Prob $>t \quad 0.9997$

Confidence $\quad 0.95$ Prob $<\mathrm{t} \quad 0.0003^{*}$

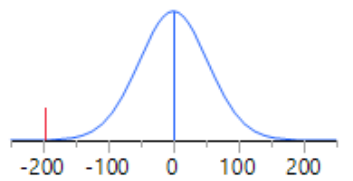

Comparing PIP with MCP

\begin{tabular}{|c|c|c|c|c|c|}
\hline Difference & 970.90 t Ratio & 18.23751 & & $A$ & \\
\hline Std Err Dif & $53.24 \mathrm{DF}$ & 48 & & & \\
\hline Upper CL Dif & 1077.94 Prob $>|t|$ & $<.0001^{\star}$ & & & \\
\hline Lower CL Dif & 863.86 Prob $>t$ & $<.0001^{\star}$ & & & \\
\hline Confidence & 0.95 Prob $<t$ & 1.0000 & $-1000-500$ & 500 & 1000 \\
\hline
\end{tabular}

Comparing PIP with Metacarpals

Difference $\quad 1167.05$ t Ratio 21.92202

$\begin{array}{lll}\text { Std Err Dif } & 53.24 \text { DF } & 48\end{array}$

Upper CL Dif 1274.09 Prob $>|t|<.0001^{*}$

Lower CL Dif 1060.01 Prob $>t \quad<.0001^{*}$

Confidence $\quad 0.95$ Prob $<\mathrm{t} \quad 1.0000$

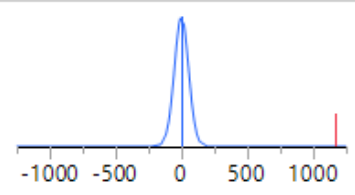




\section{Appendix C: Datasheets}

\section{Nylon Filament}

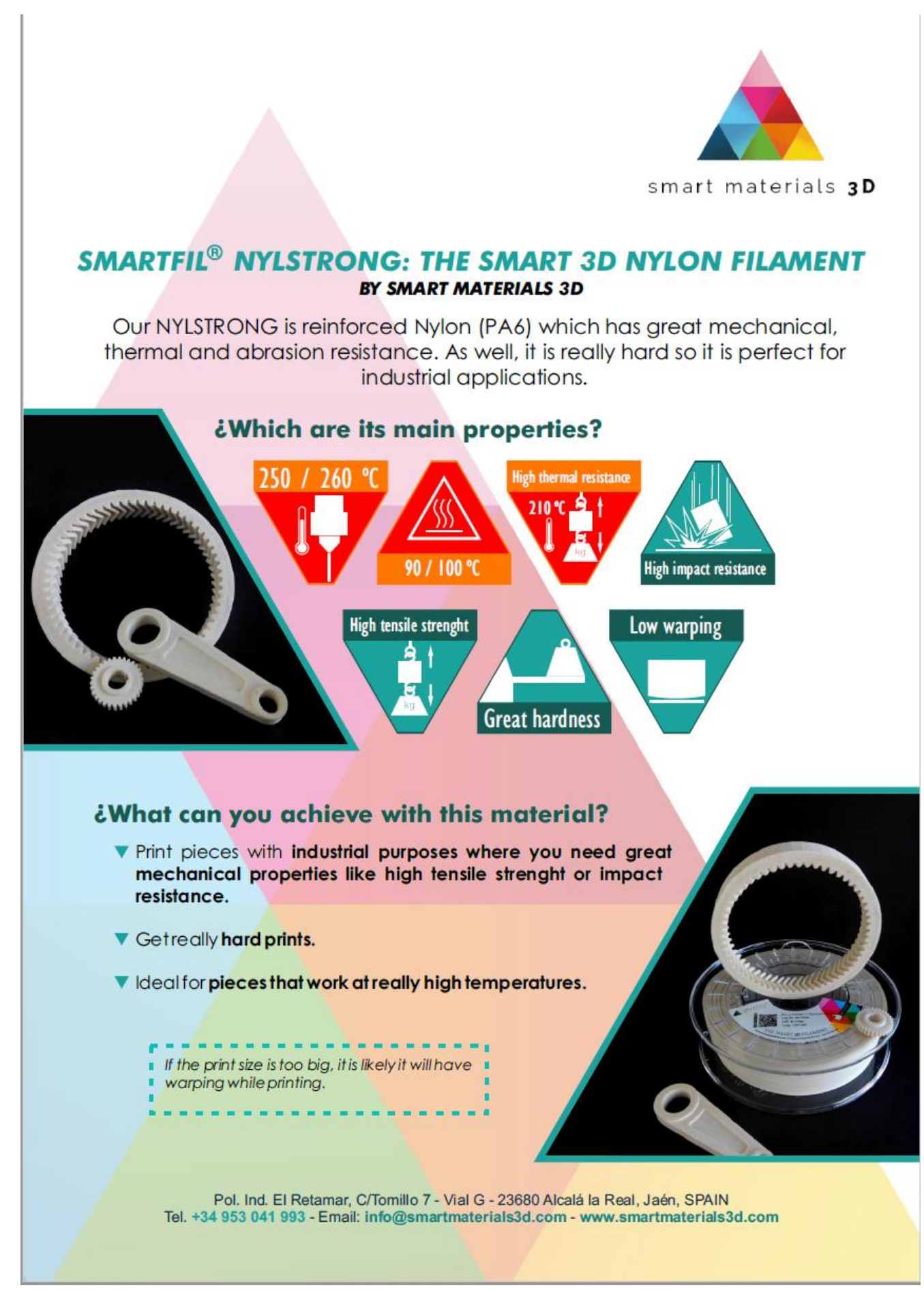




\begin{tabular}{|c|c|}
\hline \multicolumn{2}{|c|}{$\begin{array}{l}\text { SMARTFIL N NYLTRONG } \\
\text { Reinforced Nylon (PA6) which has great impact } \\
\text { resistance and a unique balance between hardness } \\
\text { and mechanical resistance. Besides, its high thermal } \\
\text { resistance makes this material perfect for industrial } \\
\text { purposes. }\end{array}$} \\
\hline WARPING & MED \\
\hline FLEXIBIUTY & $\begin{array}{l}\text { VERY } \\
\text { LOW }\end{array}$ \\
\hline BREAK RESISTANCE (IZOD) & $\begin{array}{l}\text { ULTRA } \\
\text { HIGH }\end{array}$ \\
\hline PRINT TEMPERATURE & $\pm 250^{\circ} \mathrm{C}$ \\
\hline TEMPERATURE RESISTANCE & $210^{\circ} \mathrm{C}$ \\
\hline HEATED BED & $90-110^{\circ} \mathrm{C}$ \\
\hline DENSITY & $1.52 \mathrm{~g} / \mathrm{cm}^{3}$ \\
\hline
\end{tabular}

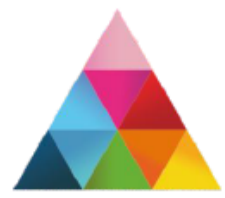

smart materials $\mathbf{3} \mathbf{D}$

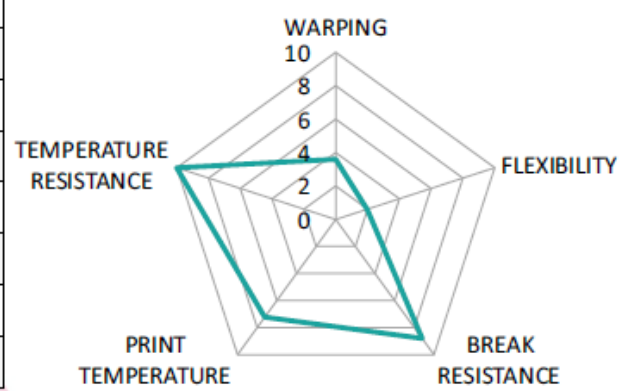

\begin{tabular}{|c|c|c|c|}
\hline CHARACTERISTIC & SMARTFIL NYLSTRONG & UNIT & TEST METHOD \\
\hline $\begin{array}{c}\text { BREAKING } \\
\text { RESISTANCE (CHARPY) }\end{array}$ & 75 & $\mathrm{KJ} / \mathrm{m}^{2}$ & $\mathrm{ISO} 179 / 1 \mathrm{eU}$ \\
\hline $\begin{array}{c}\text { TENSILE STRENGTH } \\
\text { SURFACE HARDNESS } \\
\text { (SHORED) }\end{array}$ & 612 & $\mathrm{Kg} / \mathrm{cm}^{2}$ & $1 \mathrm{SO} 527-1-2$ \\
\hline $\begin{array}{c}\text { THERMAL } \\
\text { RESISTANCE }\end{array}$ & 88 & Shore D & 1 SO 868 \\
\hline DENSITY & 1,52 & ${ }^{\circ} \mathrm{C}$ & $15075-1-2$ \\
\hline BENDING MODULUS & 1224 & $\mathrm{~g} / \mathrm{cm}^{3}$ & $1 \mathrm{ISO} 1183$ \\
\hline
\end{tabular}

DIÁMETROS

$\checkmark \quad 1.75 \mathrm{~mm}( \pm 0.03)$

$2.85 \mathrm{~mm}( \pm 0.05)$

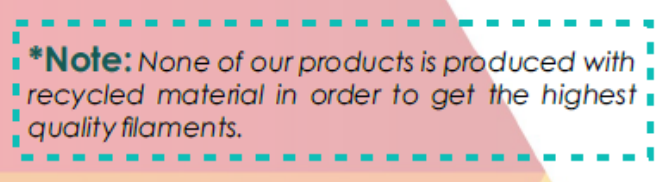

COLOURS

\begin{tabular}{|c|c|}
\hline COLOUR & NAME \\
\hline NATURAL. & NATURAL \\
\hline
\end{tabular}

PACKAGING

\begin{tabular}{|c|c|c|c|}
\hline SIZE & NET WEIGHT & GROSS WEIGHT & PACKAGING'S CHARACTERISTICS \\
\hline M & $750 \mathrm{~g}$ & $975 \mathrm{~g}$ & SMART bag, desiccant bag, guarantee seal \\
\hline
\end{tabular}

Pol. Ind. El Retamar, C/Tomillo 7 - Vial G - 23680 Alcalá la Real, Jaén, SPAIN Tel. +34953 041993 - Email: info@smartmaterials3d.com - www.smartmaterials3d.com 


\section{Glove G1}

DESCRIPTION

Thermal Plastic Rubber, Fluorescent Orange, Flexible Accordion, Spandex(B) Back/Foam Padded, Reflective Knuckle

Strip Cuff, Extended Neoprene Cuff Closure, Resists: Abrasion, Cut and Impact

\section{FEATURES}

Reinforced stitching throughout

Extra-thick smooth palm

Ergonomic design reduces hand fatigue

Machine washable

Loop to hang gloves

Adjustable wrist tab

Highly reflective knuckle strip

Thermal plastic rubber on back of fingers \& thumbs

Fluorescent orange spandex back for safety

Flexible accordion-style foam padded channels on back

Extra-thick smooth palm

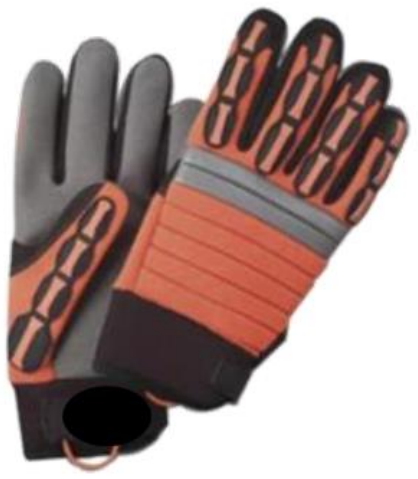

SPECIFICATIONS

$\begin{array}{ll}\text { Availability } & \text { In Stock } \\ \text { Closure Type } & \text { Extended Neoprene cuff } \\ \text { Color } & \text { Fluorescent Orange } \\ \text { Cuff Style } & \text { Reflective Knuckle Strip } \\ \text { Lining } & \text { Spandex Back/Foam Padded } \\ \text { Material } & \text { Thermal Plastic Rubber } \\ \text { Resists } & \text { Abrasion, Cut and Impact } \\ \text { Size } & \text { XL } \\ \text { Style } & \text { Flexible Accordion }\end{array}$




\section{Glove G2}

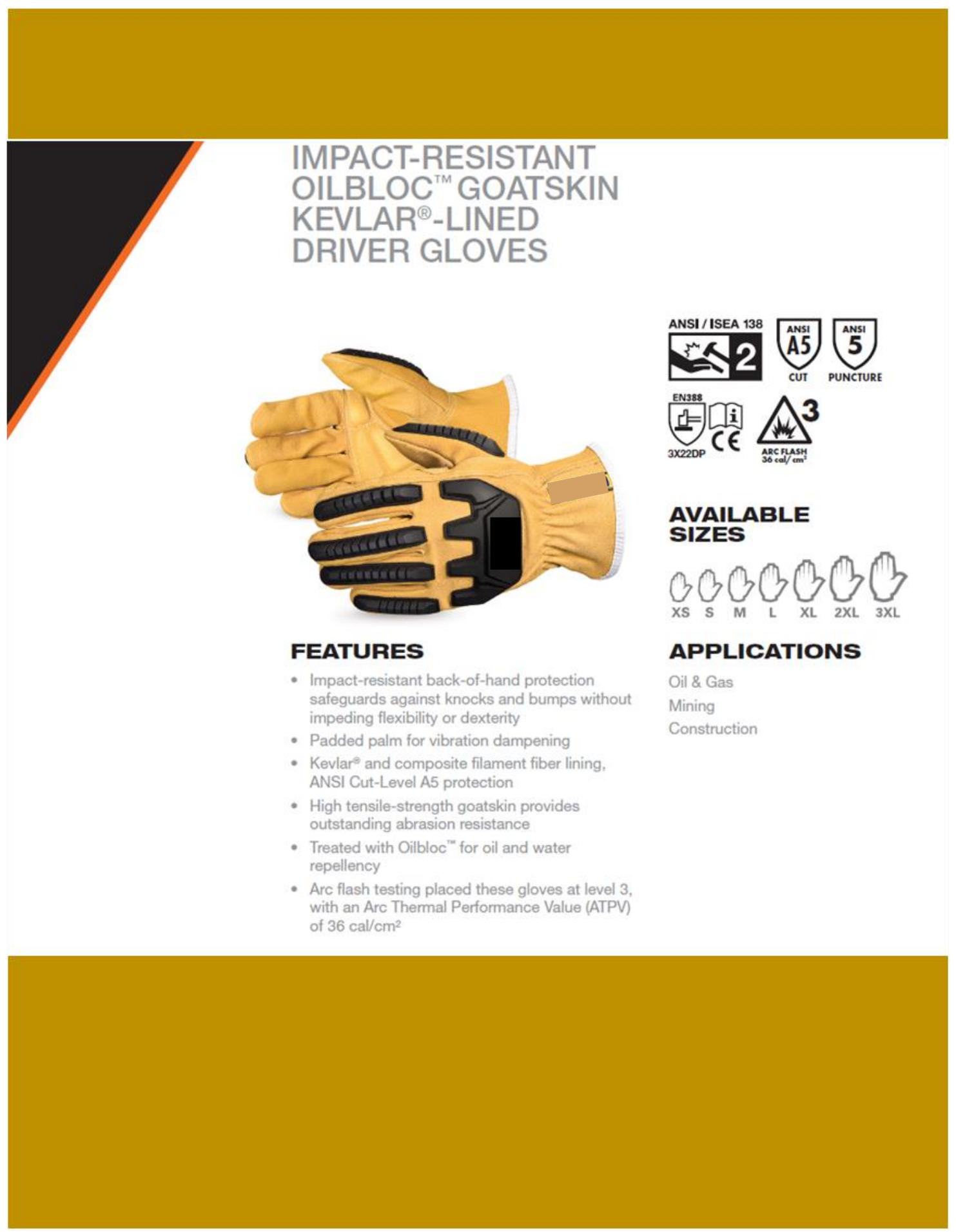




\section{Glove G3}

\section{SPEC SHEET}

PREMIUM GRAIN PIGSKIN WITH REINFORCED SPLIT PIGSKIN PALM

\section{GLOVES}

Grain pigskin leather palm, reinforced double split pigskin leather on palm, thumb and index finger, grain pigskin knuckle strap, knit wrist,

foam lining, sewn with Kevlar@ thread, canvas back, grain pigskin

leather on back of fingertips, coal miners glove

\section{FEATURES}

- Premium grain pigskin with canvas back

- Double palm, canvas back, foam lining

- Sewn with Kevlar@ thread, crisscross stitched palm

- Cut Level 2,728 g

\section{APPLICATIONS}

- Construction

- Masonry

- Agriculture

- Iron/Steel Work

- Mining

- Transportation

- Electrical Contractors

- Woodworking

\section{SPEC DETAILS}

\begin{tabular}{|l|l|}
\hline COATING & NAA \\
\hline LEATHER TYPE & Pigskin \\
\hline THUMB TYPE & Wing \\
\hline CUFF STYLE & Knit Wrist \\
\hline
\end{tabular}

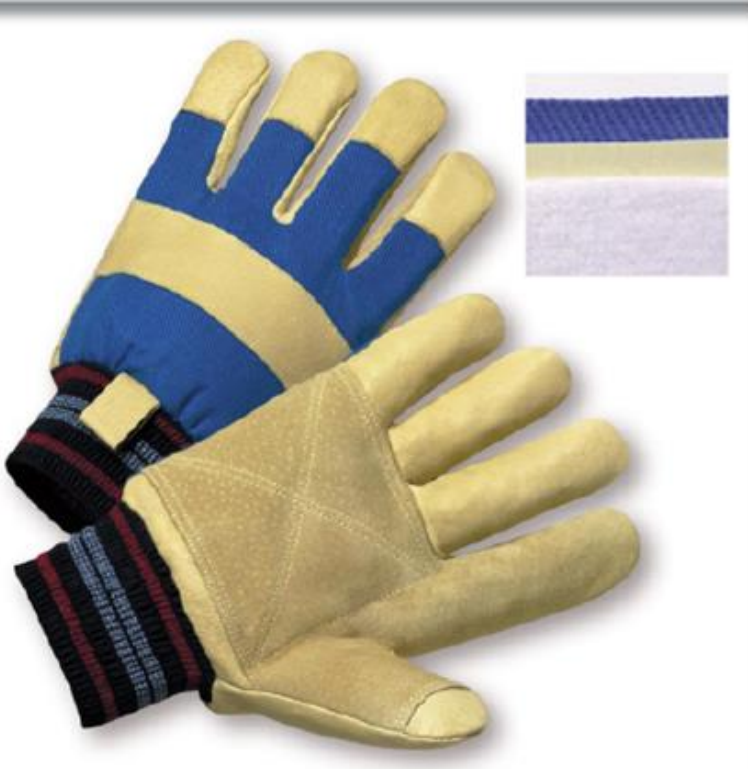

\section{TEST STANDARDS}

- Cut Level 2

$\cdot 728 \mathrm{~g}$

\section{SIZES}

Small, Medium, Large, XLarge, 2XLarge

\section{DIMENSIONS}

\begin{tabular}{|c|c|c|}
\hline ITEM SIZE & GLOVELENGTH & PALM WIDTH \\
\hline Small & $9.5^{\prime \prime}$ & $5^{\circ}$ \\
\hline Medium & $9.75^{\circ}$ & $4.75^{\prime \prime}$ \\
\hline Large & $10^{\circ}$ & $5^{\prime \prime}$ \\
\hline XLarge & $10.25^{\prime \prime}$ & $5.25^{\prime \prime}$ \\
\hline 2Xlarge & $10.5^{\prime \prime}$ & $5.5^{\prime \prime}$ \\
\hline
\end{tabular}




\section{Appendix D: Pictures}

\section{Surrogate hands and gloves}
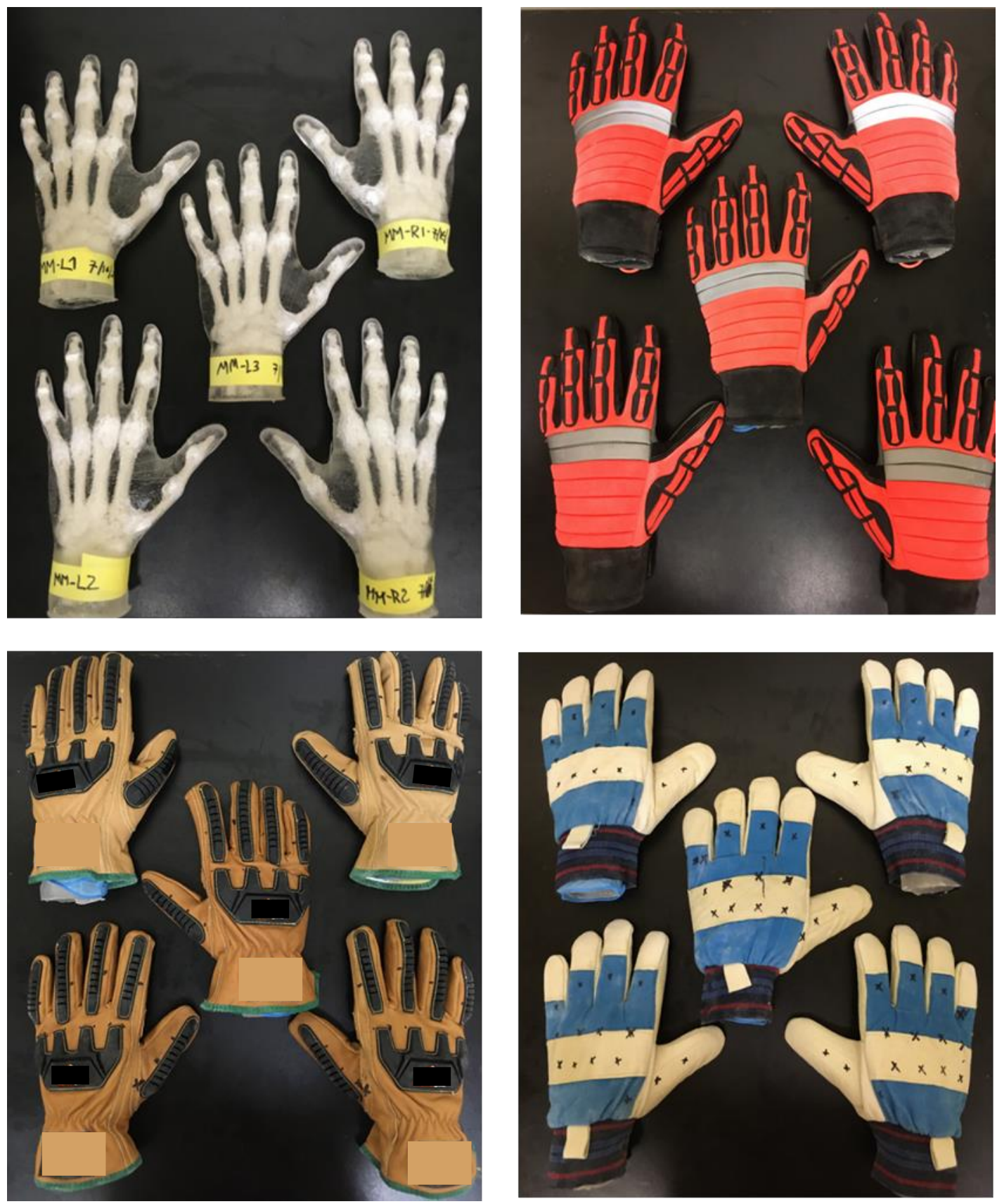

Figure A.D- 1: The different set of specimens with and without gloves used for the tests. 


\section{Surrogate hand measurements}
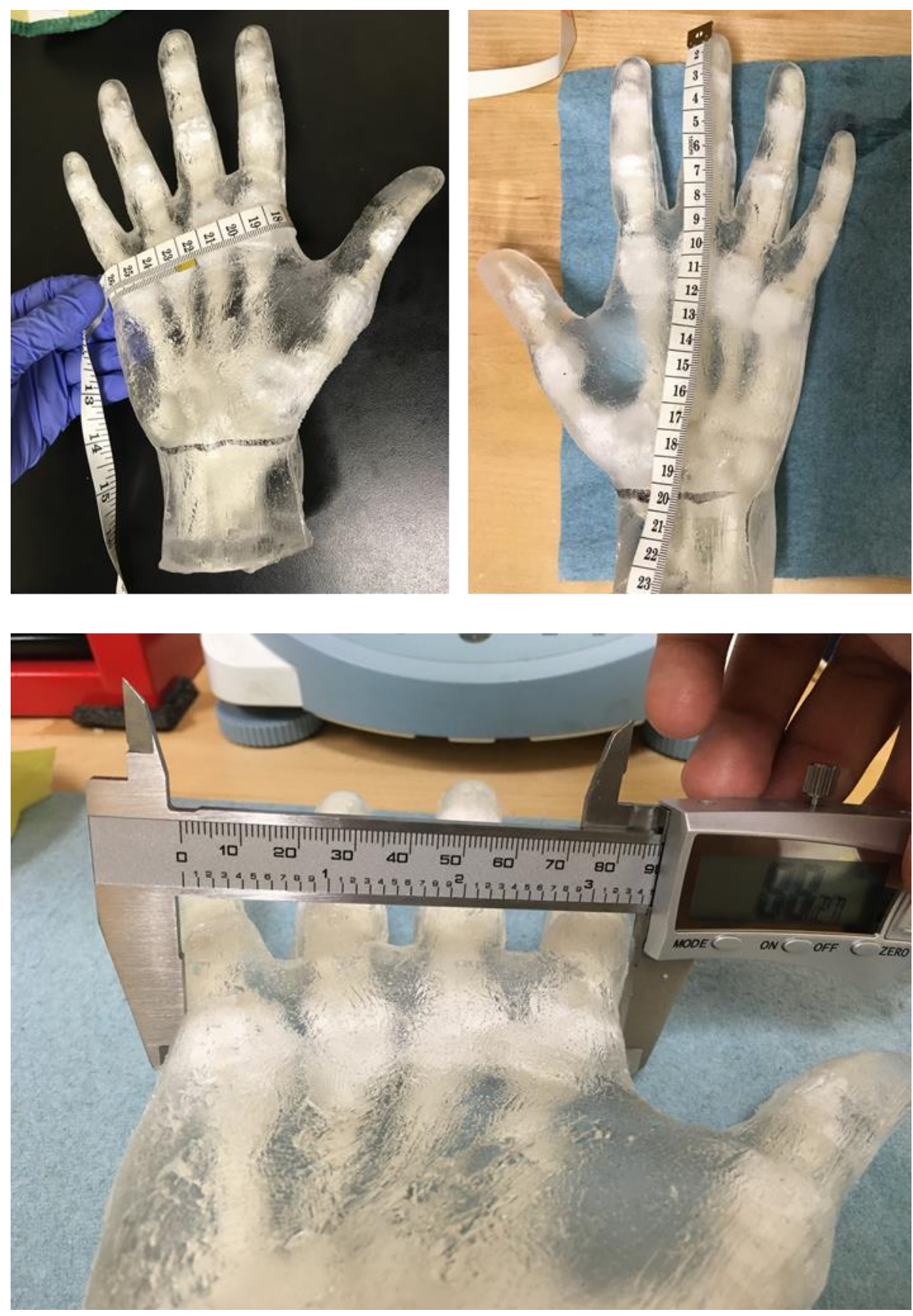

Figure A.D- 2: Measurements of circumference, length, and breadth of surrogate hands. 


\section{Impact test sample}

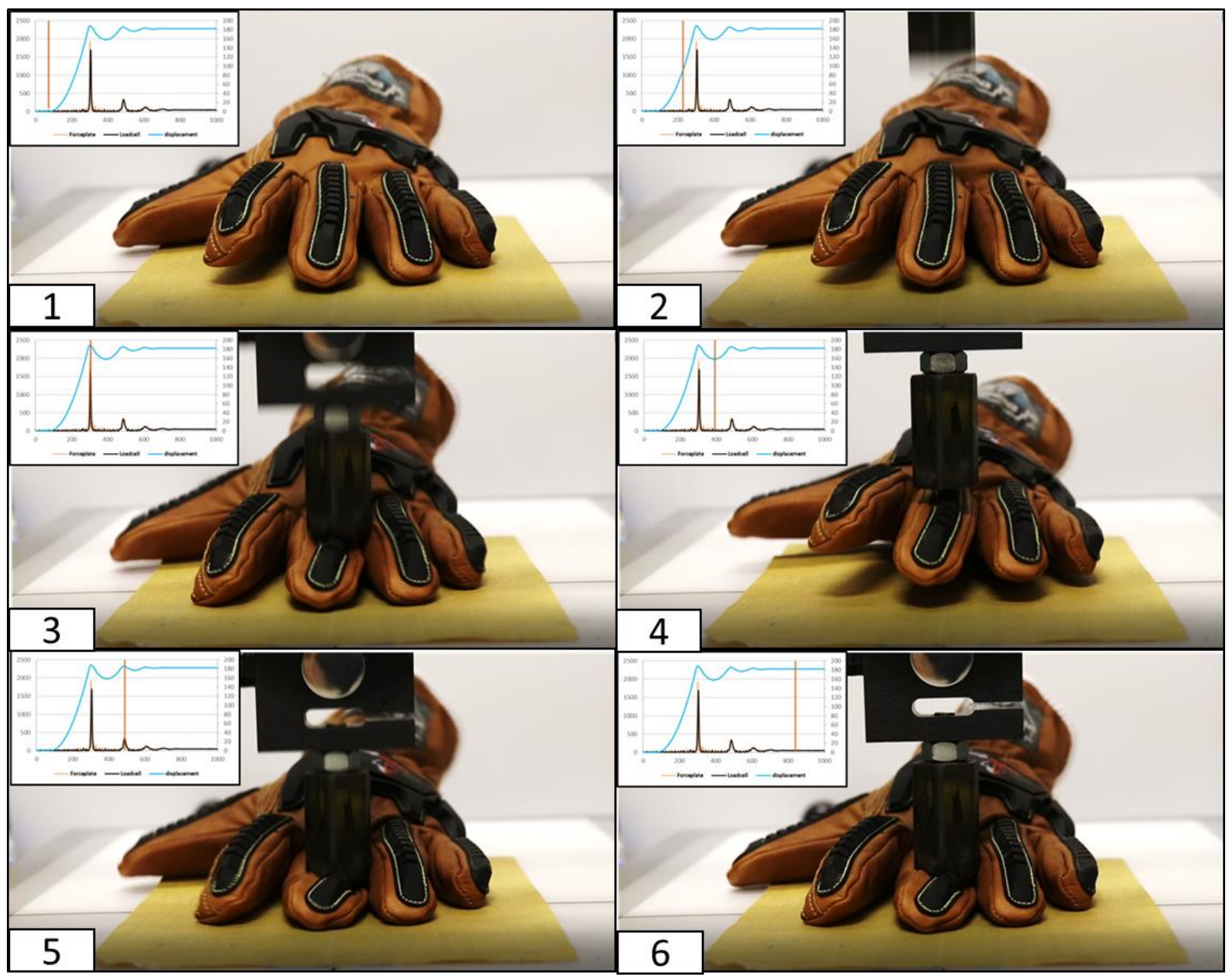

Figure A.D- 3: Snapshots of an impact test on surrogate hand with glove showing the timeline of impact and response data. 


\section{Disassembled gloves}

1. Glove G1
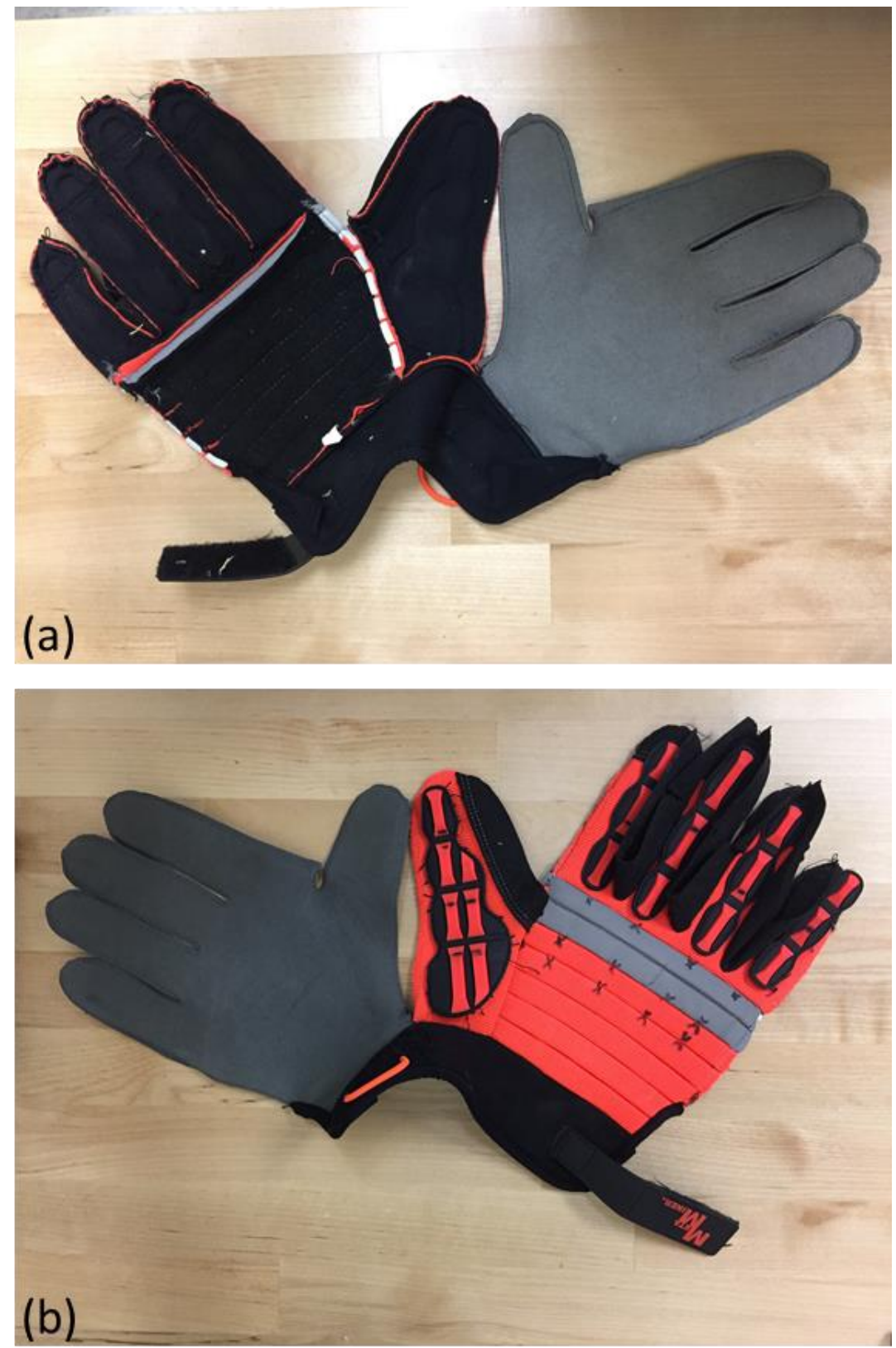

Figure A.D- 4: Disassembled glove G1. (a) Posterior \& anterior (inner). (b) Posterior \& anterior (outer). 
3. Glove G2
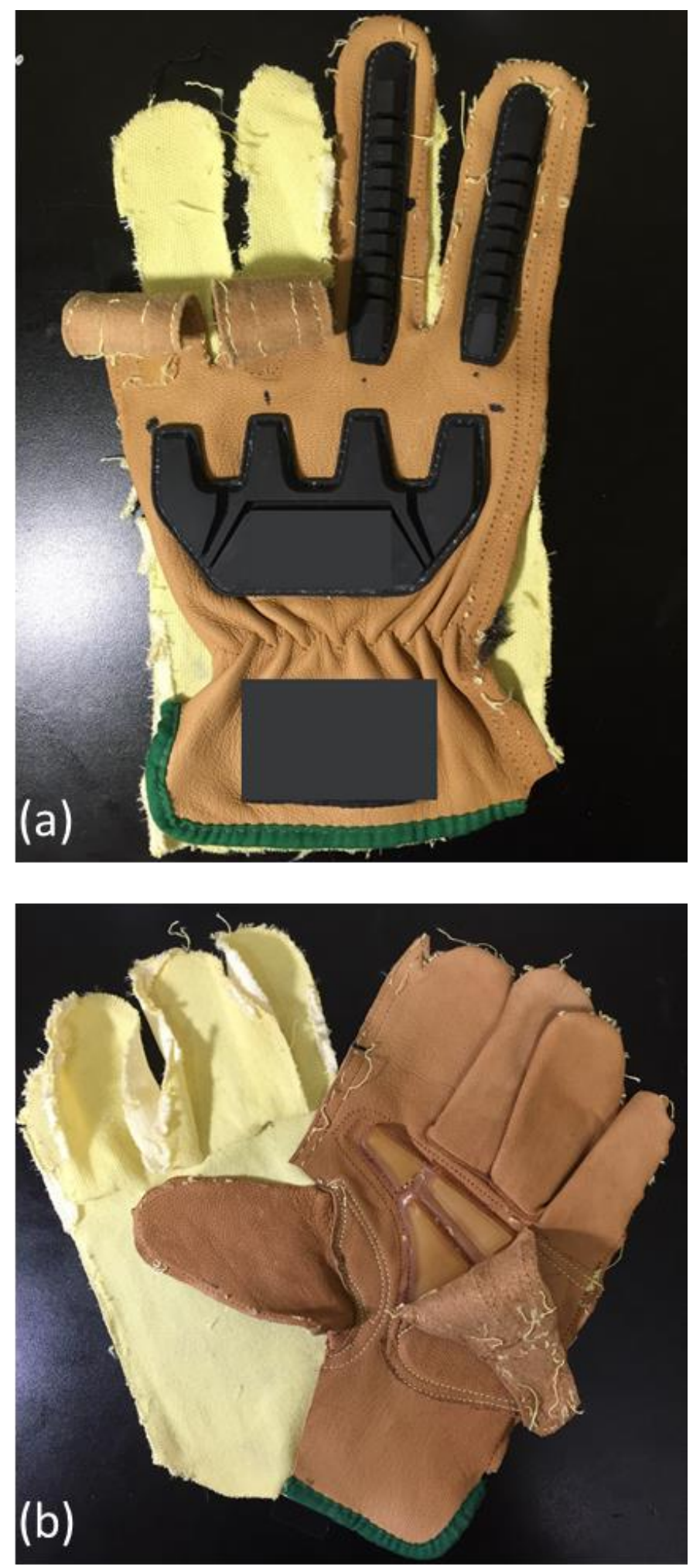

Figure A.D- 5: Disassembled glove G2. (a) Posterior. (b) Anterior. 
4. Glove G3
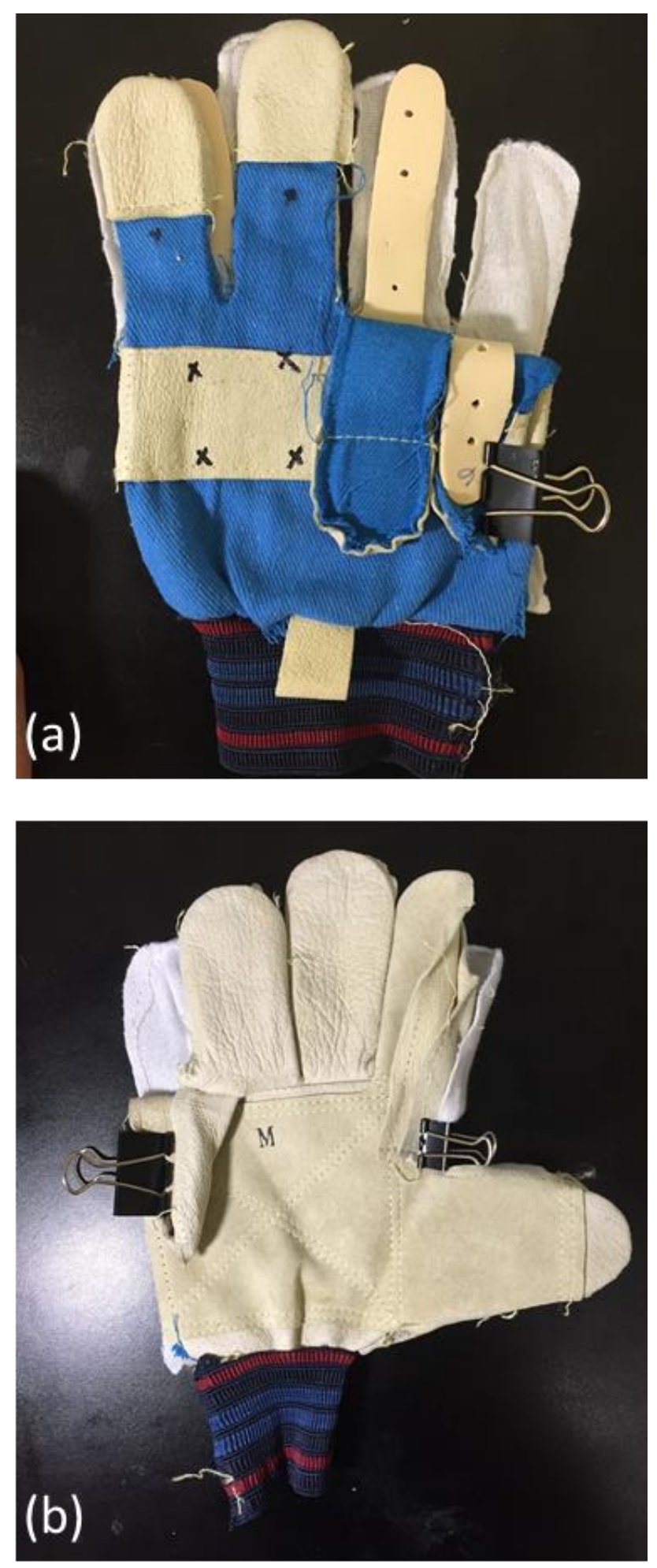

Figure A.D- 6: Disassembled glove G2. (a) Posterior. (b) Anterior. 